\title{
Null boundary terms for Lanczos-Lovelock gravity
}

\author{
Sumanta Chakraborty*1 and Krishnamohan Parattu ${ }^{\dagger 2,3,4}$ \\ ${ }^{1}$ School of Physical Sciences, Indian Association for the Cultivation of Science, Kolkata-700032, India \\ ${ }^{2}$ Instituto de Fisica, Pontificia Universidad Catolica de Valparaiso, Curauma, Valparaiso, Chile \\ ${ }^{3}$ Instituto de Ciencias Nucleares, Universidad Nacional Autónoma de México, México D.F. 04510, México \\ ${ }^{4}$ Department of Physics, IIT Madras, Chennai - 600 036, India
}

March 27, 2019

\begin{abstract}
We derive boundary terms appropriate for the general Lanczos-Lovelock action on a null boundary, when Dirichlet boundary conditions are imposed. We believe that these boundary terms have been derived for the first time in the literature. In this derivation, we rely only on the structure of the boundary variation of the action for Lanczos-Lovelock gravity. We also provide the null boundary term for Gauss-Bonnet gravity separately.
\end{abstract}

\section{Introduction}

Einstein-Hilbert action [1] is the standard action for general relativity. It is a covariant action and is constructed out of the metric and its derivatives. On varying the Einstein-Hilbert action, we obtain Einstein's field equations, which are in very good agreement with experiments and observations [2]. The Einstein-Hilbert action is unusual in that it is of second order in the derivatives of the dynamical variable of the theory, namely the metric, but has a special structure that allows the Einstein field equations to come out to be also of only second order. Since the action is of second order, one has to fix both the metric and its derivatives at the boundary surface (as the derivatives in directions tangential to the boundary are automatically fixed once we fix the metric on the boundary, we need to fix separately only the derivatives of the metric in directions non-tangential to the boundary) to kill the boundary contribution in the variation of the action. This is too much for the second order equations of motion, and there will be no solutions for most choices of the boundary conditions. We say that the action is not well-posed (see [3,4] and Chapter 6 in [5]). The ready remedy is to add boundary terms, since they modify the boundary conditions without affecting the equations of motion. A suitable boundary term needs to be added to the Einstein-Hilbert action to cancel the variation of the non-tangential derivatives of the metric on the boundary hypersurface in the variation of the action. Various proposals for such boundary terms exist in the literature but the standard one is the Gibbons-Hawking-York (GHY) boundary term $[6,7]$ (see also $[8,9]$ ). But the GHY term has the deficiency that it requires the normalized normal and non-degenerate determinant of the boundary metric, and hence can be constructed directly only for a non-null surface. A proposal for a null boundary term was first provided in [10], but it was derived in too special a case to be of much use. Much later, a null boundary term was suggested in [11], albeit for the case of a null foliation. A completely independent derivation of the null boundary term for the Einstein-Hilbert action without restricting to a foliation was presented in [12], and further refined in [13-18]. The references [13] and [17] provide treatments that do not distinguish between null and non-null boundaries (in this respect, also see [18]). When one considers non-smooth boundaries, one has to add corner terms to the gravitational action in

\footnotetext{
*sumantac.physics@gmail.com

† mailofkrishnamohan@gmail.com
} 
addition to the boundary terms. Such corner terms have been explored for both null and non-null boundaries [15, 17, 19-23].

The null boundary term has found numerous applications in various contexts. There has been quite a bit of work in the area of AdS/CFT correspondence, where the relationship between quantum complexity of states in the boundary field theory and bulk gravitational action has been explored [15, 24-38] in connection with the "complexity=action" conjecture [39,40] (see [41] for work in another direction in the area of Ads/CFT correspondence). There has also been work on formulating de Sitter spacetimes in the framework of string theory $[42,43]$. The right form of the boundary term is also important for the causal set approach to quantum gravity [44]. The topic of degrees of freedom of gravity on a null surface has also been explored [12,16, 18].

A well-known issue with the theory of general relativity is the presence of singularities, where predictability of the theory breaks down, in the context of black hole and cosmological spacetimes. One possible way to cure this issue is by supplementing the Einstein-Hilbert action with higher curvature corrections. However, diffeomorphism invariance alone is not strong enough to reasonably constrain the higher curvature candidate terms. It turns out that if one imposes an additional criterion, that the associated gravitational field equations be second order in derivatives of the metric, a very unique set of Lagrangians, including the Einstein-Hilbert Lagrangian, gets selected. These Lagrangians are known as Lanczos-Lovelock Lagrangians [45-50]. The Lanczos-Lovelock Lagrangians are perhaps the most well-motivated extensions of the Einstein-Hilbert Lagrangian. The actions for the theories in the Lanczos-Lovelock series are all second order in the derivatives of the metric, but with the equations of motion being also second order. Thus, even the Lanczos-Lovelock theories do not have a well-posed variational problem and one must add appropriate boundary terms. The non-null boundary term for the Gauss-Bonnet Lagrangian, the first non-trivial correction to the Einstein-Hilbert Lagrangian that is quadratic in the curvature tensor, was derived by Bunch [51]. For general Lanczos-Lovelock theories, the non-null boundary terms were derived by Myers [52] (see also [53-57]). However, the structure of the full boundary variation on a non-null boundary, including the total surface derivative term and the Dirichlet variation term that tells us the degrees of freedom to be fixed on the boundary and the corresponding conjugate momenta, was not known for Lanczos-Lovelock theories. We have recently derived the full structure of the non-null boundary variation starting directly from the variation of the Lanczos-Lovelock action [58] without using any topological arguments. Subsequently, the corner terms that one must add to the Lanczos-Lovelock Lagrangian in the case of non-smooth boundaries were presented in [59]. The corner terms were derived for non-null boundaries and then it was argued that the corner terms on null boundaries can be obtained as a straightforward extensions. But, to our knowledge, there have been no proposals in the literature for the boundary terms associated with the general Lanczos-Lovelock Lagrangian when the boundary surface is null in nature. In this paper, we fill this gap by explicitly deriving the boundary terms that one must add on a null boundary to make the variational principle for Lanczos-Lovelock gravity well-posed.

The procedure that we will follow will be the same as that adopted in [58]. We will start from the boundary variation of the Lanczos-Lovelock action on a null boundary and then judiciously manipulate the terms to arrive at the desired boundary term. Thus, the action itself will provide the appropriate boundary terms to be added to make it well-posed (see [60], which is perhaps the earliest work to make use of this technique). Note that one may add any term without off-theboundary derivatives of the metric (defined appropriately for null boundaries) to these boundary terms without affecting the well-posedness of the action. We attempt in this work to provide a minimal set of tensorial terms by throwing away any term without off-the-boundary derivatives of the metric.

The paper is organized as follows: We start with a very brief overview of Lanczos-Lovelock theories in Section 2. Then, we provide the various projections of the Riemann tensor near a null surface, required for our derivation, in Section 3. The formulae from these two sections are applied subsequently in Section 4 to derive the null boundary terms. After doing some preliminary simplification of the null boundary variation for Lanczos-Lovelock theories in Section 4.1, we warm up by deriving the null boundary term for the Gauss-Bonnet case in Section 4.2. Then, in Section 4.3, 
we derive the boundary term for general Lanczos-Lovelock gravity as the main result of this paper. We run a consistency check on this result in Section 4.4 by obtaining the Einstein-Hilbert and Gauss-Bonnet null boundary terms as special cases of the general result. The paper ends with a brief conclusion in Section 5. Most of the background material required for the computations have been included in the appendices.

Notations and Conventions: We use the mostly positive metric signature $(-,+,+,+, \ldots)$ and the fundamental constants $G$ and $c$ have been set to unity. The Latin indices, $a, b, \ldots$, run over all the space-time indices, and are hence summed over all the $D$ values when the spacetime is $D$-dimensional. Greek indices, $\alpha, \beta, \ldots$, will be used for indices restricted to a codimension-1 hypersurface and upper case Latin symbols, $A, B, \ldots$, will be used for indices corresponding to a codimension-2 hypersurface. We shall use the notation $R_{b c d}^{a}$ to refer to the Riemann tensor of the $D$-dimensional manifold, defined through the commutation relation $\left[\nabla_{b}, \nabla_{c}\right] v^{a}=R_{i b c}^{a} v^{i}$, and ${ }^{(D-2)} R_{b c d}^{a}$ to refer to the Riemann tensor of the codimension-2 surfaces on the null surface orthogonal to the auxiliary null vector. The $D$-dimensional covariant derivative will be denoted by $\nabla_{a}$ while the covariant derivative on the codimension- 2 surfaces will be denoted by $D_{a}$.

\section{A short summary of Lanczos-Lovelock gravity}

In this section, we shall briefly revisit the basic geometrical structure of Lanczos-Lovelock gravity, defining relevant tensors and discussing the results pertinent to our forthcoming calculations. An interested reader may consult [48] for further details and clarifications. The Lagrangian for Lanczos-Lovelock gravity is constructed as a sum over homogeneous polynomials of the Riemann curvature tensor of various orders, under the restriction that the equations of motion are second order in derivatives of the metric. For a spacetime of a particular dimension, only the terms up to a particular order are non-zero. For even number of dimensions, the highest order term is a total derivative and hence of no effect on the dynamics. Higher order terms of this series become effective as the spacetime dimension increases. For example, the Lanczos-Lovelock Lagrangian in four dimensions consists of the cosmological constant term, the Einstein-Hilbert term and the Gauss-Bonnet term. The Gauss-Bonnet term, which is the additional term present here with respect to the usual Lagrangian for general relativity, is topological in four dimensions, but can lead to non-trivial effects on the dynamics in five and higher dimensions. Thus, it is customary to consider Lanczos-Lovelock gravity in $D$ spacetime dimensions, where $D$ is greater than four, since otherwise the dynamics is effectively equal to that of general relativity with a cosmological constant term. But we are interested in the well-posedness of the action itself, and not just the dynamics. So, from our perspective, the Gauss-Bonnet term is important even in its role as a total derivative in four dimensions.

The most general action of the Lanczos-Lovelock gravity in a spacetime volume $\mathcal{V}$ in $D$ spacetime dimensions is given by

$$
16 \pi \mathcal{A}=\int d^{D} x \sqrt{-g} L=\sum_{m=0}^{m_{\max }} c_{m} \int_{\mathcal{V}} d^{D} x \sqrt{-g} L_{m} ; \quad L_{m} \equiv \frac{1}{2^{m}} \delta_{c_{1} d_{1} \ldots c_{m} d_{m}}^{a_{1} b_{1} \ldots a_{m} b_{m}} R_{a_{1} b_{1}}^{c_{1} d_{1}} \ldots R_{a_{m} b_{m}}^{c_{m} d_{m}}
$$

where $m_{\max }=[D / 2]$, i.e., the greatest integer less than or equal to $D / 2$ and $c_{m}$ 's are dimensionful coupling parameters. When $D=2 m$, the $m^{\text {th }}$ order term will be a total derivative and will not contribute to the equations of motion. The $m=0$ term in the above expression is essentially the cosmological constant term, while the $m=1$ term presents the standard Einstein-Hilbert action for the choice $c_{1}=1$. The object $\delta_{c_{1} d_{1} \ldots c_{m} d_{m}}^{a_{1} b_{1} \ldots a_{m} b_{m}}$ is the determinant of a matrix made out of delta functions and is referred to in the literature as the completely antisymmetric determinant tensor [48] or the generalized Kronecker delta [61,62]. Another tensor playing a very important role in the context of Lanczos-Lovelock theories is the entropy tensor $P_{c d}^{a b}$. Starting from the definition of the Lanczos-Lovelock Lagrangian as presented in Eq. (1), the entropy tensor takes 
the following form:

$$
P_{c d}^{a b} \equiv\left(\frac{\partial L}{\partial R_{a b}^{c d}}\right)_{g_{i j}}=\sum_{m} c_{m} \frac{m}{2^{m}} \delta_{c d c_{1} d_{1} \ldots c_{m-1} d_{m-1}}^{a b a_{1} b_{1} \ldots a_{m-1} b_{m-1}} R_{a_{1} b_{1}}^{c_{1} d_{1}} \ldots R_{a_{m-1} b_{m-1}}^{c_{m-1} d_{m-1}} \equiv \sum_{m} c_{m} P_{c d}^{a b} \quad(m)
$$

It is called the entropy tensor since it enters the expression for the Wald entropy. Using the last expression, we can write down the $m^{\text {th }}$ order Lanczos-Lovelock Lagrangian as a product of the $m^{\text {th }}$ order entropy tensor, $P_{c d(m)}^{a b}$, and the Riemann tensor:

$$
L_{m}=\frac{1}{m} P_{c d(m)}^{a b} R_{a b}^{c d}
$$

The entropy tensor $P^{a b c d}$, and also $P_{c d(m)}^{a b}$, satisfies all the algebraic symmetries of the Riemann curvature tensor. It, as well as every $P_{c d(m)}^{a b}$, also has the additional property that its divergence on any index is zero:

$$
\nabla_{a} P^{a b c d}=0
$$

This concludes our discussion of the essential geometrical structure and identities associated with the general Lanczos-Lovelock theory. Before we proceed, let us briefly comment on the two most important terms in the series for the Lanczos-Lovelock action, namely the Einstein-Hilbert (the usual action for general relativity without the cosmological constant term) and the Gauss-Bonnet terms. The first non-trivial case corresponds to $m=1$, the Einstein-Hilbert action, for which the tensor $P_{c d(1)}^{a b}$ takes the following form:

$$
P_{c d(1)}^{a b}=\frac{1}{2}\left(\delta_{c}^{a} \delta_{d}^{b}-\delta_{d}^{a} \delta_{c}^{b}\right)
$$

and the Lagrangian, as expected, is given by

$$
L_{1}=P_{c d(1)}^{a b} R_{a b}^{c d}=R
$$

The next term in the Lanczos-Lovelock Lagrangian, for $m=2$, is the the Gauss-Bonnet term, with the following expression for the entropy tensor:

$$
P_{c d(2)}^{a b}=2\left[R_{c d}^{a b}+G_{c}^{b} \delta_{d}^{a}-G_{c}^{a} \delta_{d}^{b}+R_{d}^{a} \delta_{c}^{b}-R_{d}^{b} \delta_{c}^{a}\right]
$$

The Lagrangian is

$$
L_{2}=\frac{1}{2} P_{c d(2)}^{a b} R_{a b}^{c d}=R^{2}-4 R^{a b} R_{a b}+R^{a b c d} R_{a b c d}
$$

We will require these expressions when we derive the null boundary term for Gauss-Bonnet Lagrangian in Section 4.2. Having listed the results in Lanczos-Lovelock gravity required for our work, we proceed forward to present the decompositions of the Riemann tensor near a null surface in the next section.

\section{Projections of the Riemann tensor near a null surface}

In this section, we shall provide expressions for the various projections of the Riemann tensor near a null surface that will be used in our derivation of the Lanczos-Lovelock boundary term near a null boundary. The derivations of the identities provided in this section are given in a detailed fashion in Appendix A. (For more detailed discussions on the geometrical quantities associated with null surface that are used in these derivations, we refer the reader to Appendix A.3 in the arXiv version of [12])

The identities and the results presented here are valid under the following setup. We start with a null surface in a $D$-dimensional spacetime and parametrise it by $\phi(x)=$ constant, for some scalar function $\phi(x)$. (For the purpose of this paper, we shall assume that we have taken a null foliation, 
such that the normal vector $\ell_{a}$ satisfies $\ell_{a} \ell^{a}=0$ everywhere and not just on the null boundary that we are considering.)

A null normal $\ell_{a}$ to the surface will have the form $\ell_{a}=A(x) \nabla_{a} \phi(x)$, for some scalar function $A(x)$. As is standard (see, e.g., [63,64]), we choose an auxiliary null vector $k^{a}$ such that $k^{a} \ell_{a}=-1$. This allows us to define the induced metric on the null surface as

$$
q_{a b}=g_{a b}+\ell_{a} k_{b}+k_{a} \ell_{b} .
$$

This is a two-metric on a two-surface orthogonal to $\ell^{a}$ and $k^{a}$. It turns out that it is advantageous to assume $k_{a}$ to be hyper-surface orthogonal, i.e., $k_{a}=B(x) \nabla_{a} \psi(x)$, for some scalar function $\psi(x)$ and $B(x)$. As shown in Appendix A.2, this condition is sufficient (but does not appear to be necessary) to ensure that we can define an appropriate covariant derivative $D_{a}$ on the null surface without introducing torsion. These assumptions on the null normal $\ell_{a}$ and the auxiliary null vector $k_{a}$ imply a bunch of relations that will be useful for our calculations. The "acceleration" of the null normal satisfies the following equation:

$$
\ell^{c} \nabla_{c} \ell^{d}=\kappa \ell^{d},
$$

where $\kappa$ may be called the non-affinity coefficient. The direct formula for $\kappa$ is

$$
\kappa=\ell^{a} \partial_{a} \ln A-\frac{k^{a}}{2} \partial_{a}\left(\ell^{b} \ell_{b}\right) .
$$

Since the null normal has vanishing norm everywhere, we have $\nabla_{a}\left(\ell^{b} \ell_{b}\right)=0$ and hence the nonaffinity coefficient associated with the null normal becomes

$$
\kappa=\ell^{a} \partial_{a} \ln A .
$$

The second fundamental form associated with the null surface is

$$
\Theta_{c d} \equiv q_{c}^{m} q_{d}^{k} \nabla_{m} \ell_{k}
$$

while the corresponding quantity for the null vector $k^{a}$, the second fundamental form for the foliation by $\Psi$ with $\ell^{a}$ chosen as the auxiliary null vector to $k_{a}$, is

$$
\Psi_{c d} \equiv q_{c}^{m} q_{d}^{n} \nabla_{m} k_{n} .
$$

Both $\Theta_{c d}$ and $\Psi_{c d}$ are symmetric. Finally, we have the following formula for the variation of $\ell_{a}$ when the metric is varied:

$$
\delta \ell_{a}=\ell_{a} \delta \ln A .
$$

This can be easily proved as follows: $\delta \ell_{a}=A \delta\left(\partial_{a} \phi\right)+\partial_{a} \phi \delta A=A \partial_{a}(\delta \phi)+\ell_{a} \delta \ln A=\ell_{a} \delta \ln A$, where the term $A \partial_{a}(\delta \phi)$ identically vanishes since $\phi$ is a scalar independent of the metric that does not vary under metric variations. Thus, after variation, $\ell_{a}+\delta \ell_{a}=(1+\delta \ln A) \ell_{a}=$ $(1+\delta \ln A) A \partial_{a} \phi$ is still normal to the $\phi=$ constant surfaces. In order to see how $A$ will vary under metric variations, consider a coordinate system with $\phi$ and $\psi$ as two of the coordinates. In such a coordinate system, the conditions $g^{a b} \ell_{a} \ell_{b}=0, g^{a b} k_{a} k_{b}=0$ and $g^{a b} \ell_{a} k_{b}=-1$ turn into the following conditions on the inverse metric, respectively:

$$
g^{\phi \phi}=0, \quad g^{\psi \psi}=0, \quad A B g^{\phi \psi}=-1 ;
$$

where we have used the fact that $\ell_{a}$ and $k_{a}$ have to be non-zero and hence $A$ and $B$ are non-zero. The first two conditions do not constrain $A$ or $B$, but the third condition specifies their product in terms of the metric as

$$
A B=\frac{-1}{g^{\phi \psi}} .
$$

Considering variations, it is clear that enforcing $\delta(\ell . \ell)=0$ and $\delta(\boldsymbol{k} . \boldsymbol{k})=0$ are restrictions on the metric, unlike the non-null case where $\delta(\boldsymbol{n} . \boldsymbol{n})=0$ can be achieved by varying the normalization factor of the normal appropriately. But the constraint $\delta(\boldsymbol{\ell} . \boldsymbol{k})=0$ can be achieved by tweaking 
the product $A B$ appropriately. For the purpose of this paper, we shall assume $\delta(\ell \cdot \ell)=0$ and $\delta(\boldsymbol{k} . \boldsymbol{k})=0$ everywhere, because otherwise all the expressions and equations we have for null surfaces will be meaningless under variation, and we shall also impose $\delta(\boldsymbol{\ell} . \boldsymbol{k})=0$ everywhere. [In our earlier paper deriving the null boundary term for general relativity [12], we had assumed $\delta(\ell . \ell)=0$ only on the null boundary and $\phi$ was not assumed to be a null foliation. We hope to remove these extra restrictions that we have imposed in this paper, for ease of calculation, in a later work.] We shall also assume $B$ to be a constant independent of the metric. To simplify matters, we shall equate it to 1 :

$$
B=1,
$$

which provides $A$ and the variation of $A$ in terms of the metric degrees of freedom as

$$
A=\frac{-1}{g^{\phi \psi}} \Rightarrow \delta A=\frac{\delta\left(g^{\phi \psi}\right)}{\left(g^{\phi \psi}\right)^{2}} .
$$

This is also a restriction we hope to remove in a future work.

Having described the set-up under which we will carry out the derivation of null boundary terms in the context of Lanczos-Lovelock gravity, we will now consider the projections of the Riemann tensor on the null surface that we will have occasion to use. First, we shall consider the case when all the indices of the $D$-dimensional Riemann tensor are projected on the null surface, which is given by (for a detailed derivation, see Eq. (100) in Appendix A.4)

$$
R_{c d}^{a b} q_{a}^{p} q_{b}^{q} q_{m}^{c} q_{n}^{d}={ }^{(D-2)} R_{m n}^{p q}+\Theta_{m}^{p} \Psi_{n}^{q}+\Psi_{m}^{p} \Theta_{n}^{q}-\Theta_{n}^{p} \Psi_{m}^{q}-\Psi_{n}^{p} \Theta_{m}^{q},
$$

where $\Theta_{b}^{a}$ and $\Psi_{d}^{c}$ are the second fundamental forms associated with the null normal $\ell_{a}$ and the auxiliary null vector $k_{a}$, respectively. When three indices of the Riemann tensor are projected on the null surface while the remaining index is contracted with $\ell^{a}$, one ends up with the following result (derived in Eq. (104) in Appendix A.4):

$$
R_{c d}^{a b} \ell_{a} q_{b}^{m} q_{p}^{c} q_{q}^{d}=D_{q} \Theta_{p}^{m}-D_{p} \Theta_{q}^{m}+\left(q_{q}^{i} \Theta_{p}^{m}-q_{p}^{i} \Theta_{q}^{m}\right) k^{j} \nabla_{i} \ell_{j} .
$$

And finally, when two indices of the Riemann tensor are projected on the null surface and the other two are contracted with the null normal $\ell^{a}$, we get

$$
R_{c d}^{a b} \ell_{a} \ell^{c} q_{b}^{m} q_{n}^{d}=-q_{i}^{m} q_{n}^{j} £_{\ell} \Theta_{j}^{i}+\kappa \Theta_{n}^{m}-\Theta_{i}^{m} \Theta_{n}^{i} .
$$

For a derivation of the above equation, we refer the reader to Eq. (115) in Appendix A.4. Note that the left-hand side of this equation is a symmetric tensor and each of the terms on the righthand side are also symmetric. These are all the projections we can have without making use of $k^{a}$, since a term with three indices of the Riemann tensor contracted with the null normal $\ell_{a}$ would identically vanish due to the symmetries of the Riemann tensor.

\section{The null boundary term for Lanczos-Lovelock gravity}

\subsection{Manipulating the boundary variation in general Lanczos-Lovelock gravity}

In this section, we shall start with the total divergence term in the variation of the action of general Lanczos-Lovelock gravity, express it as the boundary variation on a null boundary and separate out the total variation term to be cancelled by the addition of a boundary term. The expression for the total divergence term in the action of Lanczos-Lovelock gravity, following [5], is

$$
\delta \mathcal{A}_{\partial \mathcal{V}}=\int_{\mathcal{V}} d^{D} x \sqrt{-g} \nabla_{j}\left[2 P^{i b j d} \nabla_{b}\left(\delta g_{d i}\right)-2\left(\nabla_{c} P^{i j c d}\right) \delta g_{d i}\right] .
$$

Enforcing Eq. (4), i.e., the result that the contracted covariant derivative of the entropy tensor $P^{a b c d}$ identically vanishes, one can eliminate the second term appearing in the above expression. 
We use the divergence theorem to convert the remaining expression from a volume integral to a boundary integral on the null boundary with normal $\ell_{a}$, leading to

$$
\delta \mathcal{A}_{\partial \mathcal{V}}=\int_{\partial \mathcal{V}} d^{D-1} x 2\left(\frac{\sqrt{-g}}{A}\right) \ell_{j} P^{i b j d} \nabla_{b}\left(\delta g_{d i}\right),
$$

where the form of the volume element used is for $\ell_{a}=A \nabla_{a} \phi$ with $\phi$ taken as one of the coordinates [12]. In Appendix D, we have derived the following relation between $\sqrt{-g}$ and the determinant $q$ of the metric on the codimension-2 surfaces:

$$
\sqrt{-g}=A \sqrt{q}
$$

Using this relation in Eq. (24) we obtain

$$
\delta \mathcal{A}_{\partial \mathcal{V}}=\int_{\partial \mathcal{V}} d^{D-1} x 2 \sqrt{q} \ell_{j} P^{i b j d} \nabla_{b}\left(\delta g_{d i}\right) .
$$

Let us lose the integral and just write the integrand appearing above:

$$
\begin{aligned}
\sqrt{q} Q\left[\ell_{c}\right] & \equiv 2 \sqrt{q} \ell_{c} P^{a b c d} \nabla_{b}\left(\delta g_{a d}\right) \\
& =2 \sqrt{q}\left[\nabla_{b}\left(P^{a b c d} \ell_{c} \delta g_{a d}\right)-P^{a b c d}\left(\nabla_{b} \ell_{c}\right) \delta g_{a d}\right] .
\end{aligned}
$$

The last term is a Dirichlet variation term (a term with only the variations of the dynamical variables, and not of their derivatives) and it will be killed when we fix the metric on the boundary. (There is the question of which components of the metric are to be fixed on the boundary for a consistent variational principle. Let us assume for now that, just as in the non-null case [58], the decomposition of the boundary term will automatically select the relevant components and ensure consistency.) Since this does not contribute to the boundary term, let us ignore it and write

$$
\sqrt{q} Q\left[\ell_{c}\right]=2 \sqrt{q} \nabla_{b}\left(P^{a b c d} \ell_{c} \delta g_{a d}\right)+\ldots .
$$

From now on, any term deemed not relevant for the boundary term will be dumped into the '...'. Decomposing the first term using the projector $\Pi_{b}^{a}=\delta_{b}^{a}+k^{a} \ell_{b}$ (see [13]), we obtain

$$
\begin{aligned}
2 \sqrt{q} \nabla_{b}\left(P^{a b c d} \ell_{c} \delta g_{a d}\right) & =2 \frac{\sqrt{-g}}{A} \nabla_{b}\left(P^{a b c d} \ell_{c} \delta g_{a d}\right)=2 \sqrt{-g} \nabla_{b}\left(\frac{1}{A} P^{a b c d} \ell_{c} \delta g_{a d}\right)+\ldots \\
& =2 \sqrt{-g} \nabla_{e}\left(\frac{1}{A} \Pi^{e}{ }_{b} P^{a b c d} \ell_{c} \delta g_{a d}\right)-2 \sqrt{-g} \nabla_{e}\left(\frac{1}{A} k^{e} \ell_{b} P^{a b c d} \ell_{c} \delta g_{a d}\right)+\ldots \\
& =2 \partial_{e}\left(\frac{\sqrt{-g}}{A} \Pi^{e}{ }_{b} P^{a b c d} \ell_{c} \delta g_{a d}\right)-2 \sqrt{-g} \nabla_{e}\left(\frac{1}{A} k^{e} \ell_{b} P^{a b c d} \ell_{c} \delta g_{a d}\right)+\ldots
\end{aligned}
$$

Since $\Pi^{e}{ }_{b} \ell_{e}=0$, the first term above is a surface total derivative term on the null boundary. This term has variations of the derivatives of the metric, but all these derivatives are tangential to the boundary and hence will disappear when the metric is fixed all along the boundary. Thus, this term does not contribute to the boundary term. Relegating it also to the oblivion of the three dots, we obtain

$$
\sqrt{q} Q\left[\ell_{c}\right]=-2 \sqrt{q} \nabla_{e}\left(k^{e} \ell_{b} P^{a b c d} \ell_{c} \delta g_{a d}\right)+\ldots .
$$

Note that, as we have done above, we can take $A$ inside and outside of the derivative as we see fit, since the extra terms are anyway proportional to $\delta g_{a b}$ and hence do not contribute to the boundary term. Taking the covariant derivative in Eq. (30) inside, we obtain

$$
\sqrt{q} Q\left[\ell_{c}\right]=-2 \sqrt{q} \nabla_{e}\left(k^{e} \ell_{b} P^{a b c d} \ell_{c}\right) \delta g_{a d}-2 \sqrt{q} k^{e} \ell_{b} P^{a b c d} \ell_{c} \nabla_{e} \delta g_{a d}+\ldots
$$

Again, we spy a Dirichlet variation term in the above expression. Relegating it to the dots, we can write

$$
\sqrt{q} Q\left[\ell_{c}\right]=-2 \sqrt{q} k^{e} \ell_{b} P^{a b c d} \ell_{c} \nabla_{e} \delta g_{a d}+\ldots
$$


Next, we need to bring the $\delta$ out of the covariant derivative in $\nabla_{e} \delta g_{a d}$. We can manipulate this term as follows:

$$
\begin{aligned}
\nabla_{e} \delta g_{a d} & =\partial_{e} \delta g_{a d}-\Gamma_{e a}^{f} \delta g_{f d}-\Gamma_{e d}^{f} \delta g_{a f} \\
& =\partial_{e} \delta g_{a d}-\delta\left(\Gamma_{e a}^{f} g_{f d}\right)-\delta\left(\Gamma_{e d}^{f} g_{a f}\right)+g_{f d} \delta \Gamma_{e a}^{f}+g_{a f} \delta \Gamma_{e d}^{f} \\
& =\delta\left(\nabla_{e} g_{a d}\right)+g_{f d} \delta \Gamma_{e a}^{f}+g_{a f} \delta \Gamma_{e d}^{f}=g_{f d} \delta \Gamma_{e a}^{f}+g_{a f} \delta \Gamma_{e d}^{f} .
\end{aligned}
$$

We have converted the variations of the metric and its derivatives to variations of the Christoffel symbols. In Eq. (32), the index $e$ above is contracted with the auxiliary null vector $k^{e}$. We shall use the following identity to transform these terms:

$$
k^{e} \delta \Gamma_{e a}^{f}=\delta\left(\nabla_{a} k^{f}\right)-\nabla_{a}\left(\delta k^{f}\right) .
$$

Using Eq. (33) and Eq. (34) in Eq. (32), we obtain

$$
\begin{aligned}
-2 \sqrt{q} k^{e} \ell_{b} P^{a b c d} \ell_{c} \nabla_{e} \delta g_{a d} & =2 \sqrt{q} k^{e} P^{a b d c} \ell_{b} \ell_{c}\left(g_{f d} \delta \Gamma_{e a}^{f}+g_{a f} \delta \Gamma_{e d}^{f}\right) \\
& =4 \sqrt{q} k^{e} P^{a b d c} \ell_{b} \ell_{c} g_{f d} \delta \Gamma_{e a}^{f} \\
& =4 \sqrt{q} P^{a b d c} \ell_{b} \ell_{c} g_{f d}\left[\delta\left(\nabla_{a} k^{f}\right)-\nabla_{a}\left(\delta k^{f}\right)\right] .
\end{aligned}
$$

Decomposing the second term involving $\nabla_{a} \delta k^{f}$ above,

$$
\begin{aligned}
-4 \sqrt{q} P^{a b d c} \ell_{b} \ell_{c} g_{f d} \nabla_{a}\left(\delta k^{f}\right)= & -4 \sqrt{q} P^{a b d c} \ell_{b} \ell_{c} g_{f d}\left(\Pi^{e}{ }_{a}-k^{e} \ell_{a}\right) \nabla_{e}\left[\delta k^{f}\right] \\
= & -4 \sqrt{q} P^{a b d c} \ell_{b} \ell_{c} g_{f d} \Pi^{e}{ }_{a} \nabla_{e}\left[\delta k^{f}\right] \\
= & -4 \sqrt{q} \nabla_{e}\left[\Pi^{e}{ }_{a} P^{a b d c} \ell_{b} \ell_{c} g_{f d} \delta k^{f}\right] \\
& +4 \sqrt{q} \nabla_{e}\left[P^{a b d c} \ell_{b} \ell_{c} g_{f d} \Pi^{e}{ }_{a}\right] \delta k^{f}
\end{aligned}
$$

where we have used the antisymmetry of indices $a, b$ in $P^{a b c d}$ in the second step. The second term involving $\delta k^{f}$ is a Dirichlet variation term, since our assumption $k_{a}=\nabla_{a} \psi$ means that $\delta k^{f}=k_{a} \delta g^{f a}$. As in Eq. (29), the first term can be converted to a total derivative term on the boundary surface by taking the $\sqrt{q}$ inside the covariant derivative at the cost of appearance of a Dirichlet variation term. Thus, consigning all the terms in Eq. (36) to the dots, we have

$$
\begin{aligned}
\sqrt{q} Q\left[\ell_{c}\right] & =4 \sqrt{q} P^{a b d c} \ell_{b} \ell_{c} g_{f d} \delta\left(\nabla_{a} k^{f}\right)+\ldots \\
& =4 \sqrt{q} P^{a b c d} \ell_{a} \ell_{c} g_{f d} \delta\left(\nabla_{b} k^{f}\right)+\ldots \\
& =4 \sqrt{q} P_{c d}^{a b} \ell_{a} \ell^{c}\left(\tilde{\Pi}_{f}^{d}-\ell^{d} k_{f}\right)\left(\Pi_{b}^{m}-k^{m} \ell_{b}\right) \delta\left(\nabla_{m} k^{f}\right)+\ldots \\
& =4 \sqrt{q} P_{c d}^{a b} \ell_{a} \ell^{c} \tilde{\Pi}_{f}{ }^{d} \Pi^{m} \delta\left(\nabla_{m} k^{f}\right)+\ldots
\end{aligned}
$$

where we have introduced the notation $\tilde{\Pi}_{a}{ }^{b}=\delta_{a}^{b}+k_{a} \ell^{b}$. This is just $\Pi_{b}^{a}$ with the first index lowered and the second one raised. But we have added the tilde so that it will be easier to recognize this without looking minutely at index placements.

Dropping the $\sqrt{q}$ factor for the time being for ease, the boundary variation can be further manipulated as follows:

$$
\begin{aligned}
Q\left[\ell_{c}\right]= & 4 P_{c d}^{a b} \ell_{a} \ell^{c} \Pi^{m}{ }_{b} \tilde{\Pi}_{n}^{d} \delta\left(\nabla_{m} k^{n}\right)+\ldots \\
= & 4 P_{c d}^{a b} \ell_{a} \ell^{c}\left(q_{b}^{m}-\ell^{m} k_{b}\right)\left(q_{n}^{d}-\ell_{n} k^{d}\right) \delta\left(\nabla_{m} k^{n}\right)+\ldots \\
= & 4 P_{c d}^{a b} \ell_{a} \ell^{c} q_{b}^{m} q_{n}^{d} \delta\left(\nabla_{m} k^{n}\right)-4 P_{c d}^{a b} \ell_{a} \ell^{c} q_{b}^{m} \ell_{n} k^{d} \delta\left(\nabla_{m} k^{n}\right)-4 P_{c d}^{a b} \ell_{a} \ell^{c} \ell^{m} k_{b} q_{n}^{d} \delta\left(\nabla_{m} k^{n}\right) \\
& +4 P_{c d}^{a b} \ell_{a} \ell^{c} \ell^{m} k_{b} k^{d} \ell_{n} \delta\left(\nabla_{m} k^{n}\right)+\ldots \\
= & 4 P_{c d}^{a b} \ell_{a} \ell^{c} \delta \Psi_{b}^{d}-4 P_{c d}^{a b} \ell_{a} \ell^{c} k^{d} q_{b}^{m} \delta\left(\ell_{n} \nabla_{m} k^{n}\right)-4 P_{c d}^{a b} \ell_{a} \ell^{c} k_{b} q_{n}^{d} \delta\left(\ell^{m} \nabla_{m} k^{n}\right) \\
& +4 P_{c d}^{a b} \ell_{a} \ell^{c} k_{b} k^{d} \delta\left(\ell^{m} \ell_{n} \nabla_{m} k^{n}\right)+\ldots \\
= & 4 P_{c d}^{a b} \ell_{a} \ell^{c} \delta \Psi_{b}^{d}+4 P_{c d}^{a b} \ell_{a} \ell^{c} k^{d} q_{b}^{m} \delta\left(k^{p} \nabla_{m} \ell_{p}\right)+4 P_{c d}^{a b} \ell_{a} \ell^{c} k_{b} q_{n}^{d} \delta\left(k^{p} \nabla^{n} \ell_{p}\right)
\end{aligned}
$$




$$
\begin{aligned}
& +4 P_{c d}^{a b} \ell_{a} \ell^{c} k_{b} k^{d} \delta \kappa+\ldots \\
= & 4 P_{c d}^{a b} \ell_{a} \ell^{c} \delta \Psi_{b}^{d}+8 P_{c d}^{a b} \ell_{a} \ell^{c} k^{d} q_{b}^{m} \delta\left(k^{p} \nabla_{m} \ell_{p}\right)+4 P_{c d}^{a b} \ell_{a} \ell^{c} k_{b} k^{d} \delta \kappa+\ldots .
\end{aligned}
$$

In the above derivation, we have replaced $\Pi$ and $\tilde{\Pi}$ in terms of the induced metric $q_{b}^{a}$ and simplified the resultant expressions. Any terms involving metric variations $\left(\delta \ell^{a}, \delta k^{a}, \delta q_{b}^{a}, \delta g^{a b}\right.$, etc.) have been banished to the anonymity of the three dots and we have made use of the equations Eq. (10) and Eq. (14). Further, we have used the assumption that $\ell_{a} k^{a}=-1$ everywhere and even under variations to write

$$
\ell_{n} \nabla_{m} k^{n}=\nabla_{m}\left(\ell_{n} k^{n}\right)-k^{n} \nabla_{m} \ell_{n}=\partial_{m}\left(\ell_{n} k^{n}\right)-k^{n} \nabla_{m} \ell_{n}=-k^{n} \nabla_{m} \ell_{n} .
$$

The simplification of the term $-4 P_{c d}^{a b} \ell_{a} \ell^{c} k_{b} q_{n}^{d} \delta\left(\ell^{m} \nabla_{m} k^{n}\right)$ was performed using

$$
\ell^{m} \nabla_{m} k^{n}=\ell^{m} \nabla_{m} \nabla^{n} \psi=\ell^{m} \nabla^{n} \nabla_{m} \psi=\ell^{m} \nabla^{n} k_{m}=-k^{m} \nabla^{n} \ell_{m},
$$

to write

$$
-4 P_{c d}^{a b} \ell_{a} \ell^{c} k_{b} q_{n}^{d} \delta\left(\ell^{m} \nabla_{m} k^{n}\right)=4 P_{c d}^{a b} \ell_{a} \ell^{c} k_{b} q_{n}^{d} \delta\left(k^{m} \nabla^{n} \ell_{m}\right)+\ldots,
$$

which was then combined with another term using the symmetries of $P^{a b c d}$ to obtain Eq. (38).

We will use Eq. (38) to evaluate the structure of the boundary term for the general LanczosLovelock Lagrangian. But first, as a warmup and due to its significance as the first higher order correction to Einstein gravity, we consider the case of the Gauss-Bonnet term.

\subsection{Specializing to Gauss-Bonnet gravity}

Before deriving the boundary term for general Lanczos-Lovelock gravity, let us briefly discuss the boundary term structure associated with Gauss-Bonnet gravity, i.e., with the $m=2$ term in the Lanczos-Lovelock series. The expression for the entropy tensor $P_{c d}^{a b}$ in the context of Gauss-Bonnet gravity was given in Eq. (7), which we reproduce below for convenience:

$$
P_{c d(2)}^{a b}=2\left(R_{c d}^{a b}+G_{c}^{b} \delta_{d}^{a}-G_{c}^{a} \delta_{d}^{b}+R_{d}^{a} \delta_{c}^{b}-R_{d}^{b} \delta_{c}^{a}\right) .
$$

Using the above formula for the entropy tensor and Eq. (38), we shall derive the boundary term in Gauss-Bonnet gravity. We shall simplify each of the terms in Eq. (38) in turn. The first among them is

$$
\begin{aligned}
Q_{\mathrm{GB}}^{(1)}\left[\ell_{c}\right] & \equiv 4 P_{c d(2)}^{a b} \ell_{a} \ell^{c} \delta \Psi_{b}^{d} \\
& =4 P_{c d(2)}^{a b} \ell_{a} \ell^{c} q_{b}^{m} q_{n}^{d} \delta\left(\nabla_{m} k^{n}\right)+\ldots \\
& =8\left(R_{c d}^{a b} \ell_{a} \ell^{c} q_{b}^{m} q_{n}^{d}-G_{c}^{a} \ell_{a} \ell^{c} q_{n}^{m}\right) \delta\left(\nabla_{m} k^{n}\right)+\ldots \\
& =8\left(R_{c d}^{a b} \ell_{a} \ell^{c} q_{b}^{m} q_{n}^{d}-R_{c}^{a} \ell_{a} \ell^{c} q_{n}^{m}\right) \delta\left(\nabla_{m} k^{n}\right)+\ldots \\
& =8\left[\left(-q_{a}^{m} q_{n}^{b} £_{\ell} \Theta_{b}^{a}+\kappa \Theta_{n}^{m}-\Theta_{p}^{m} \Theta_{n}^{p}\right)-\left(\kappa \Theta-\frac{d \Theta}{d \lambda}-\Theta_{b}^{a} \Theta_{a}^{b}\right) q_{n}^{m}\right] \delta\left(\nabla_{m} k^{n}\right)+\ldots \\
& =8\left(-q_{a}^{m} q_{n}^{b} £_{\ell} \Theta_{b}^{a}+\kappa \Theta_{n}^{m}-\Theta_{p}^{m} \Theta_{n}^{p}\right) \delta \Psi_{m}^{n}-8\left(\kappa \Theta-\frac{d \Theta}{d \lambda}-\Theta_{b}^{a} \Theta_{a}^{b}\right) \delta \Psi+\ldots
\end{aligned}
$$

where we have used Eq. (22) to substitute for $R_{c d}^{a b} \ell_{a} \ell^{c} q_{b}^{m} q_{n}^{d}$ and also used

$$
R_{c}^{a} \ell_{a} \ell^{c}=\kappa \Theta-\frac{d \Theta}{d \lambda}-\Theta_{b}^{a} \Theta_{a}^{b} .
$$

Here we have introduced a parameter $\lambda$ such that $\ell^{c} \nabla_{c} \lambda=1$ to write $\ell^{c} \nabla_{c} \Theta=(d \Theta / d \lambda)$. Eq. (44) is the null Raychaudhuri equation [64], but with non-affine parametrisation [65]. In fact, we can derive this equation easily from the projections of the Riemann tensor. We have given this derivation in Appendix F. 
The next term in Eq. (38) is

$$
\begin{aligned}
Q_{\mathrm{GB}}^{(2)}\left[\ell_{c}\right] & \equiv 8 P_{c d(2)}^{a b} \ell_{a} \ell^{c} k^{d} q_{b}^{m} \delta\left(k^{p} \nabla_{m} \ell_{p}\right) \\
& =16\left(R_{c d}^{a b} \ell_{a} \ell^{c} k^{d} q_{b}^{m}-G_{c}^{b} \ell^{c} q_{b}^{m}\right) \delta\left(k^{p} \nabla_{m} \ell_{p}\right) \\
& =16 R_{c d}^{a b} \ell^{c} q_{b}^{m}\left(\ell_{a} k^{d}+\delta_{a}^{d}\right) \delta\left(k^{p} \nabla_{m} \ell_{p}\right) \\
& =16 R_{c d}^{a b} \ell^{c} q_{b}^{m}\left(q_{a}^{d}-k_{a} \ell^{d}\right) \delta\left(k^{p} \nabla_{m} \ell_{p}\right) \\
& =-16 R_{c d}^{a b} \ell^{c} q_{a}^{m} q_{b}^{d} \delta\left(k^{p} \nabla_{m} \ell_{p}\right) \\
& =-16\left[-D^{m} \Theta+D_{p} \Theta^{m p}-\left(\Theta q^{a m}-\Theta^{m a}\right) k^{p} \nabla_{a} \ell_{p}\right] \delta\left(k^{q} \nabla_{m} \ell_{q}\right),
\end{aligned}
$$

where we have used a contracted version of Eq. (21) in arriving at the last step. The remaining term in Eq. (38) has the following structure

$$
\begin{aligned}
Q_{\mathrm{GB}}^{(3)}\left[\ell_{c}\right] & \equiv 4 P_{c d(2)}^{a b} \ell_{a} \ell^{c} k_{b} k^{d} \delta \kappa=8\left(R_{c d}^{a b} \ell_{a} \ell^{c} k_{b} k^{d}-G_{c}^{b} \ell^{c} k_{b}-R_{d}^{a} \ell_{a} k^{d}\right) \delta \kappa \\
& =8\left(R_{c d}^{a b} \ell_{a} \ell^{c} k_{b} k^{d}-2 R_{d}^{a} \ell_{a} k^{d}-\frac{1}{2} R\right) \delta \kappa=-4 R_{c d}^{a b} q_{a}^{c} q_{b}^{d} \delta \kappa \\
& =-4\left({ }^{(D-2)} R+2 \Theta \Psi-2 \Theta_{a b} \Psi^{a b}\right) \delta \kappa
\end{aligned}
$$

where, in the penultimate step, we have used the following result:

$$
R_{c d}^{a b} q_{a}^{c} q_{b}^{d}=R+4 R_{b}^{a} \ell_{a} k^{b}-2 R_{c d}^{a b} \ell_{a} \ell^{c} k_{b} k^{d},
$$

and contracted version of Eq. (20) in the ultimate step. Finally, combining Eq. (43), Eq. (45) and Eq. (46), we arrive at

$$
\begin{aligned}
Q_{\mathrm{GB}}\left[\ell_{c}\right] \equiv & Q_{\mathrm{GB}}^{(1)}\left[\ell_{c}\right]+Q_{\mathrm{GB}}^{(2)}\left[\ell_{c}\right]+Q_{\mathrm{GB}}^{(3)}\left[\ell_{c}\right] \\
= & -8\left(q_{p}^{m} q_{n}^{q} £_{\ell} \Theta_{m}^{n}\right) \delta \Psi_{q}^{p}+8 \kappa \Theta_{n}^{m} \delta \Psi_{m}^{n}-8 \Theta_{p}^{m} \Theta_{n}^{p} \delta \Psi_{m}^{n}-8 \kappa \Theta \delta \Psi+8 \frac{d \Theta}{d \lambda} \delta \Psi \\
& +8 \Theta_{b}^{a} \Theta_{a}^{b} \delta \Psi+16 D^{m} \Theta \delta\left(k^{q} \nabla_{m} \ell_{q}\right)-16 D_{p} \Theta^{m p} \delta\left(k^{q} \nabla_{m} \ell_{q}\right) \\
& +16\left(k^{q} \nabla_{a} \ell_{q}\right)\left(\Theta q^{a m}-\Theta^{a m}\right) \delta\left(k^{p} \nabla_{m} \ell_{p}\right)-4^{(D-2)} R \delta \kappa-8 \Theta \Psi \delta \kappa+8 \Theta_{a b} \Psi^{a b} \delta \kappa+\ldots \\
= & -8\left(q_{p}^{m} q_{n}^{q} £_{\ell} \Theta_{m}^{n}\right) \delta \Psi_{q}^{p}+8 \Theta_{n}^{m} \delta\left(\kappa \Psi_{m}^{n}\right)-8 \Theta_{p}^{m} \Theta_{n}^{p} \delta \Psi_{m}^{n}-8 \Theta \delta(\kappa \Psi)+8 \frac{d \Theta}{d \lambda} \delta \Psi \\
& +8 \Theta_{b}^{a} \Theta_{a}^{b} \delta \Psi+16 D^{m} \Theta \delta\left(k^{q} \nabla_{m} \ell_{q}\right)-16 D_{p} \Theta^{m p} \delta\left(k^{q} \nabla_{m} \ell_{q}\right) \\
& +8\left(\Theta q^{a m}-\Theta^{a m}\right) \delta\left[\left(k^{q} \nabla_{a} \ell_{q}\right)\left(k^{p} \nabla_{m} \ell_{p}\right)\right]-4^{(D-2)} R \delta \kappa+\ldots,
\end{aligned}
$$

where we have again thrown some metric variations to the dots. All these terms have variations of the derivatives of the metric orthogonal to the null surface, i.e., derivatives along $k^{a}$. Taking the delta commonly outside and eliminating the terms with only variations of the metric or its surface derivatives on the boundary to the group of terms represented by the dots, we obtain

$$
\begin{aligned}
Q_{\mathrm{GB}}\left[\ell_{c}\right]=\delta[ & -8 \Psi_{q}^{p} £_{\ell} \Theta_{p}^{q}+8 \kappa \Theta_{q}^{p} \Psi_{p}^{q}-8 \Theta_{c}^{a} \Theta_{b}^{c} \Psi_{a}^{b}-8 \kappa \Theta \Psi+8 \Psi \frac{d \Theta}{d \lambda}+8 \Psi \Theta_{b}^{a} \Theta_{a}^{b} \\
& +16\left(D^{m} \Theta-D^{a} \Theta_{a}^{m}\right)\left(k^{q} \nabla_{m} \ell_{q}\right)+8\left(k^{q} \nabla_{m} \ell_{q}\right)\left(k^{p} \nabla_{a} \ell_{p}\right)\left(\Theta q^{m a}-\Theta^{m a}\right) \\
& \left.-4 \kappa{ }^{(D-2)} R\right]+\ldots,
\end{aligned}
$$

where we have used the fact that $\Theta^{a b}, D_{c}$ acting on $\Theta^{a b}, \Theta, q_{p}^{m} q_{n}^{q} £_{\ell} \Theta_{m}^{n}, d \Theta / d \lambda$ and ${ }^{(D-2)} R$ do not have derivatives of the metric along $k^{a}$. (From Eq. (92), one can see that ${ }^{(D-2)} R$ contains only derivatives tangential to the null surface.) Introducing back the $\sqrt{q}$ which we had dropped before Eq. (38), we have

$$
\begin{aligned}
\sqrt{q} Q_{\mathrm{GB}}\left[\ell_{c}\right]=\delta\{\sqrt{q}[ & -8 \Psi_{q}^{p} £_{\ell} \Theta_{p}^{q}+8 \kappa \Theta_{q}^{p} \Psi_{p}^{q}-8 \Theta_{c}^{a} \Theta_{b}^{c} \Psi_{a}^{b}-8 \kappa \Theta \Psi+8 \Psi \frac{d \Theta}{d \lambda}+8 \Psi \Theta_{b}^{a} \Theta_{a}^{b} \\
& +16\left(D^{m} \Theta-D^{a} \Theta_{a}^{m}\right)\left(k^{q} \nabla_{m} \ell_{q}\right)+8\left(k^{q} \nabla_{m} \ell_{q}\right)\left(k^{p} \nabla_{a} \ell_{p}\right)\left(\Theta q^{m a}-\Theta^{m a}\right)
\end{aligned}
$$




$$
\left.\left.-4 \kappa^{(D-2)} R\right]\right\}+\ldots
$$

where the variations of $\sqrt{q}$ have been removed to the dots. Therefore, the boundary term to be added to cancel off the normal derivatives of the metric in the boundary variation for Gauss-Bonnet gravity is

$$
\begin{aligned}
\sqrt{q} \mathcal{B}_{\mathrm{GB}}=4 \sqrt{q} & {\left[2 \Psi_{q}^{p} £_{\ell} \Theta_{p}^{q}-2 \kappa \Theta_{q}^{p} \Psi_{p}^{q}+2 \Theta_{c}^{a} \Theta_{b}^{c} \Psi_{a}^{b}+2 \kappa \Theta \Psi-2 \Psi \frac{d \Theta}{d \lambda}-2 \Psi \Theta_{b}^{a} \Theta_{a}^{b}\right.} \\
& -4\left(D^{m} \Theta-D^{a} \Theta_{a}^{m}\right)\left(k^{q} \nabla_{m} \ell_{q}\right)-2\left(k^{q} \nabla_{m} \ell_{q}\right)\left(k^{p} \nabla_{a} \ell_{p}\right)\left(\Theta q^{m a}-\Theta^{m a}\right) \\
& \left.+\kappa^{(D-2)} R\right] .
\end{aligned}
$$

Grouping terms with $\kappa$, we can rewrite this boundary term as

$$
\begin{array}{r}
\sqrt{q} \mathcal{B}_{\mathrm{GB}}=4 \sqrt{q}\left[\kappa\left({ }^{(D-2)} R-2 \Theta_{q}^{p} \Psi_{p}^{q}+2 \Theta \Psi\right)+2 \Psi_{q}^{p} £_{\ell} \Theta_{p}^{q}-2 \Psi \frac{d \Theta}{d \lambda}+2 \Theta_{c}^{a} \Theta_{b}^{c} \Psi_{a}^{b}-2 \Psi \Theta_{b}^{a} \Theta_{a}^{b}\right. \\
\left.-4\left(D^{m} \Theta-D^{a} \Theta_{a}^{m}\right)\left(k^{q} \nabla_{m} \ell_{q}\right)-2\left(k^{q} \nabla_{m} \ell_{q}\right)\left(k^{p} \nabla_{a} \ell_{p}\right)\left(\Theta q^{m a}-\Theta^{m a}\right)\right] .
\end{array}
$$

This finishes our computation of the null boundary term in Gauss-Bonnet gravity. This boundary term is considerably larger than the null boundary term for Einstein-Hilbert action $[11,12,15]$. The derivatives of the metric in directions non-tangential to the null boundary are present in the tensors $\kappa, \Psi_{a b}$ and $k^{p} \nabla_{a} \ell_{p}$, while only $\kappa$ was present in the Einstein-Hilbert null boundary term.

We will now take up our main task, that of finding out the boundary term for general LanczosLovelock gravity.

\subsection{Boundary term for general Lanczos-Lovelock gravity}

In this section, we shall derive the null boundary term for general Lanczos-Lovelock gravity by proceeding on a path similar to the one used in the last section to successfully derive the null boundary term in the context of Gauss-Bonnet gravity.

We shall derive the null boundary term for $m^{\text {th }}$ order Lanczos-Lovelock gravity. The null boundary term for any Lanczos-Lovelock action can be obtained as a suitable sum of these terms. The expression of the entropy tensor $P_{c d}^{a b}$ for $m^{\text {th }}$ order Lanczos-Lovelock gravity takes the following form (see Eq. (2)):

$$
P_{c d(m)}^{a b}=\frac{m}{2^{m}} \delta_{c d c_{1} d_{1} \ldots c_{m-1} d_{m-1}}^{a b a_{1} b_{1} \ldots a_{m-1} b_{m-1}} R_{a_{1} b_{1}}^{c_{1} d_{1}} \ldots R_{a_{m-1} b_{m-1}}^{c_{m-1} d_{m-1}}
$$

To make the index notation less cumbersome, we shall do the evaluation for $(m+1)^{t h}$ order Lanczos-Lovelock gravity, since then we will have $m$ instead of $(m-1)$ in the last set of indices presented above and the expressions will be slightly shorter.

\subsubsection{Projecting and decomposing the Riemann tensors in the boundary varia- tion}

In Eq. (38), we start with the last term, involving $\delta \kappa$, which is the simplest to work with. We multiply this term by $\left[2^{m+1} /(m+1)\right]$ to remove the corresponding factor from $P_{c d(m+1)}^{a b}$ to obtain

$$
\begin{aligned}
\frac{2^{m+1}}{m+1} Q_{m+1}^{(3)}\left[\ell_{c}\right] & \equiv \frac{2^{m+1}}{m+1}\left[4 P_{c d(m+1)}^{a b} \ell_{a} \ell^{c} k_{b} k^{d} \delta \kappa\right] \\
& =4 \delta \kappa \ell_{a} \ell^{c} k_{b} k^{d} \delta_{c d r_{1} s_{1} \ldots r_{m} s_{m}}^{a b p_{1} q_{1} \ldots p_{m} q_{m}} R_{p_{1} q_{1}}^{r_{1} s_{1}} \ldots R_{p_{m} q_{m}}^{r_{m} s_{m}}
\end{aligned}
$$




$$
\begin{aligned}
= & 4 \delta \kappa \ell_{a} \ell^{c} k_{b} k^{d} \delta_{c d r_{1} s_{1} \ldots r_{m} s_{m}}^{a b p_{1} q_{1} \ldots p_{m} q_{m}}\left(q_{p_{1}}^{a_{1}} q_{q_{1}}^{b_{1}} q_{c_{1}}^{r_{1}} q_{d_{1}}^{s_{1}} R_{a_{1} b_{1}}^{c_{1} d_{1}}\right) \ldots\left(q_{p_{m}}^{a_{m}} q_{q_{m}}^{b_{m}} q_{c_{m}}^{r_{m}} q_{d_{m}}^{s_{m}} R_{a_{m} b_{m}}^{c_{m} d_{m}}\right) \\
= & 4 \delta \kappa \ell_{a} \ell^{c} k_{b} k^{d} \delta_{c d r_{1} s_{1} \ldots r_{m} s_{m}}^{a b p_{1} q_{1} \ldots p_{m} q_{m}}\left({ }^{(D-2)} R_{p_{1} q_{1}}^{r_{1} s_{1}}+4 \Theta_{p_{1}}^{r_{1}} \Psi_{q_{1}}^{s_{1}}\right) \ldots\left({ }^{(D-2)} R_{p_{m} q_{m}}^{r_{m} s_{m}}+4 \Theta_{p_{m}}^{r_{m}} \Psi_{q_{m}}^{s_{m}}\right) \\
= & 4 \delta \kappa \ell_{a} \ell^{c} k_{b} k^{d} \delta_{c d r_{1} s_{1} \ldots r_{m} s_{m}}^{a b p_{1} q_{1} \ldots p_{m} q_{m}} \\
& \times \sum_{t=0}^{m}{ }^{m} C_{t} 4^{t}\left({ }^{(D-2)} R_{p_{1} q_{1}}^{r_{1} s_{1}} \ldots{ }^{(D-2)} R_{p_{m-t} q_{m-t}}^{r_{m-t} s_{m-t}}\right)\left(\Theta_{p_{m-t+1}}^{r_{m-t+1}} \Psi_{q_{m-t+1}}^{s_{m-t+1}} \ldots \Theta_{p_{m}}^{r_{m}} \Psi_{q_{m}}^{s_{m}}\right),
\end{aligned}
$$

where, in the third step, we have written the Kronecker delta as $\delta_{b}^{a}=q_{b}^{a}-\ell^{a} k_{b}-\ell_{b} k^{a}$ and have used the antisymmetry of the determinant tensor. In the fourth line, we have made use of Eq. (20) to write down the projected $D$-dimensional Riemann tensor in terms of the induced Riemann tensor and $\Theta_{a b}$ and $\Psi_{a b}$. In the next step, we have expanded out the product of the $m$ factors. Each term in the sum involves $m$ factors, the factors present being either the components of the induced Riemann tensor or factors of the form $4 \Theta_{b}^{a} \Psi_{d}^{c}$. All terms having the same number of Riemann tensor factors are equivalent due to the symmetries of $\delta_{c d r_{1} s_{1} \ldots r_{m} s_{m}}^{a b p_{1} q_{1} \ldots p_{m}}$. The binomial coefficient ${ }^{m} C_{t}$ represents the number of ways of choosing $k$ factors out of the $m$ factors present. It is to be understood that the indices are to be taken only from the pool $\left(p_{1}, q_{1}, r_{1}, s_{1}\right)$ to $\left(p_{m}, q_{m}, r_{m}, s_{m}\right)$, with each index appearing only once. For example, the $t=0$ term is built from the induced Riemann tensor alone, while the $t=m$ term depends solely on factors inheriting $\Theta_{b}^{a}$ and $\Psi_{b}^{a}$.

Next, we tackle the second term in Eq. (38) involving the variation of $k^{p} \nabla_{m} \ell_{p}$, again multiplying by $\left[2^{m+1} /(m+1)\right]$ :

$$
\begin{aligned}
& \frac{2^{m+1}}{m+1} Q_{m+1}^{(2)}\left[\ell_{c}\right] \equiv \frac{2^{m+1}}{m+1}\left[8 P_{c d(m+1)}^{a b} \ell_{a} \ell^{c} k^{d} q_{b}^{n} \delta\left(k^{p} \nabla_{n} \ell_{p}\right)\right] \\
& =8 \ell_{a} \ell^{c} k^{d} q_{b}^{n} \delta\left(k^{p} \nabla_{n} \ell_{p}\right) \delta_{c d r_{1} s_{1} \ldots r_{m} s_{m}}^{a b p_{1} q_{1} \ldots p_{m} q_{m}} R_{p_{1} q_{1}}^{r_{1} s_{1}} \ldots R_{p_{m} q_{m}}^{r_{m} s_{m}} \\
& =8 \ell_{a} \ell^{c} k^{d} q_{b}^{n} \delta\left(k^{p} \nabla_{n} \ell_{p}\right) \delta_{c d r_{1} s_{1} \ldots r_{m} s_{m}}^{a b p_{1} q_{1} \ldots p_{m} q_{m}}\left(q_{p_{1}}^{a_{1}} q_{q_{1}}^{b_{1}} q_{c_{1}}^{r_{1}} q_{d_{1}}^{s_{1}} R_{a_{1} b_{1}}^{c_{1} d_{1}}\right) \ldots\left(q_{p_{m}}^{a_{m}} q_{q_{m}}^{b_{m}} q_{c_{m}}^{r_{m}} q_{d_{m}}^{s_{m}} R_{a_{m} b_{m}}^{c_{m} d_{m}}\right) \\
& -16 m \ell_{a} \ell^{c} k^{d} q_{b}^{n} \delta\left(k^{p} \nabla_{n} \ell_{p}\right) \delta_{c d r_{1} s_{1} r_{2} s_{2} \ldots r_{m} s_{m}}^{a b p_{1} q_{1} p_{2} q_{2} \ldots p_{m} q_{m}} k_{p_{1}}\left(\ell^{a_{1}} q_{q_{1}}^{b_{1}} q_{c_{1}}^{r_{1}} q_{d_{1}}^{s_{1}} R_{a_{1} b_{1}}^{c_{1} d_{1}}\right) \\
& \times\left(q_{p_{2}}^{a_{2}} q_{q_{2}}^{b_{2}} q_{c_{2}}^{r_{2}} q_{d_{2}}^{s_{2}} R_{a_{2} b_{2}}^{c_{2} d_{2}}\right) \ldots\left(q_{p_{m}}^{a_{m}} q_{q_{m}}^{b_{m}} q_{c_{m}}^{r_{m}} q_{d_{m}}^{s_{m}} R_{a_{m} b_{m}}^{c_{m} d_{m}}\right)
\end{aligned}
$$

where, again, the decomposition of the Kronecker delta as $\delta_{b}^{a}=q_{b}^{a}-\ell^{a} k_{b}-\ell_{b} k^{a}$ and the symmetry properties of the determinant tensor were used. Since none of the upper indices of the determinant tensor are a priori contracted with a $k_{a}$, terms of the form $k_{b} \ell^{c}$ arising from the expansion of $\delta_{b}^{c}$ survive when the index $b$ is contracted with the determinant tensor and the index $c$ is contracted with a lower index of one of the Riemann tensors, with all the other Riemann indices present, of that particular Riemann tensor and of others in the product, contracted with $q_{b}^{a}$. All such terms are equivalent due to the symmetries of the determinant tensor. Since there are two lower indices per Riemann tensor and there are $m$ Riemann tensors to choose from, we get an overall factor of $2 m$ for this term. We shall first simplify the first term in the Eq. (55), where all the indices of the Riemann tensors are contracted with $q$ 's:

$$
\begin{aligned}
\frac{2^{m+1}}{m+1} Q_{m+1}^{(2, a)}\left[\ell_{c}\right] \equiv & 8 \ell_{a} \ell^{c} k^{d} q_{b}^{n} \delta\left(k^{p} \nabla_{n} \ell_{p}\right) \delta_{c d r_{1} s_{1} \ldots r_{m} s_{m}}^{a b p_{1} q_{1} \ldots p_{m} q_{m}}\left(q_{p_{1}}^{a_{1}} q_{q_{1}}^{b_{1}} q_{c_{1}}^{r_{1}} q_{d_{1}}^{s_{1}} R_{a_{1} b_{1}}^{c_{1} d_{1}}\right) \ldots\left(q_{p_{m}}^{a_{m}} q_{q_{m}}^{b_{m}} q_{c_{m}}^{r_{m}} q_{d_{m}}^{s_{m}} R_{a_{m} b_{m}}^{c_{m} d_{m}}\right) \\
= & 8 \ell_{a} \ell^{c} k^{d} q_{b}^{n} \delta\left(k^{p} \nabla_{n} \ell_{p}\right) \delta_{c r_{1} s_{1} \ldots r_{m} s_{m}}^{a b p_{1} q_{1} \ldots p_{m} q_{m}} \\
& \times \sum_{t=0}^{m}{ }^{m} C_{t} 4^{t}\left({ }^{(D-2)} R_{p_{1} q_{1}}^{r_{1} s_{1}} \ldots{ }^{(D-2)} R_{p_{m-t} q_{m-t}}^{r_{m} s_{m-t}}\right)\left(\Theta_{p_{m-t+1}}^{r_{m-t+1}} \Psi_{q_{m-t+1}}^{s_{m-t+1}} \ldots \Theta_{p_{m}}^{r_{m}} \Psi_{q_{m}}^{s_{m}}\right)
\end{aligned}
$$

where we have made use of Eq. (20) and expanded just as in Eq. (54). Going on to the second term in Eq. (55), we have

$$
\frac{2^{m+1}}{m+1} Q_{m+1}^{(2, b)}\left[\ell_{c}\right] \equiv-16 m \ell_{a} \ell^{c} k^{d} q_{b}^{n} \delta\left(k^{p} \nabla_{n} \ell_{p}\right) \delta_{c d r_{1} s_{1} r_{2} s_{2} \ldots r_{m} s_{m}}^{a b p_{1} q_{1} p_{2} q_{2} \ldots p_{m} q_{m}} k_{p_{1}}\left(\ell^{a_{1}} q_{q_{1}}^{b_{1}} q_{c_{1}}^{r_{1}} q_{d_{1}}^{s_{1}} R_{a_{1} b_{1}}^{c_{1} d_{1}}\right)
$$




$$
\begin{aligned}
& \quad \times\left(q_{p_{2}}^{a_{2}} q_{q_{2}}^{b_{2}} q_{c_{2}}^{r_{2}} q_{d_{2}}^{s_{2}} R_{a_{2} b_{2}}^{c_{2} d_{2}}\right) \ldots\left(q_{p_{m}}^{a_{m}} q_{q_{m}}^{b_{m}} q_{c_{m}}^{r_{m}} q_{d_{m}}^{s_{m}} R_{a_{m} b_{m}}^{c_{m} d_{m}}\right) \\
& =-32 m \ell_{a} \ell^{c} k_{b} k^{d} \delta\left(q_{p_{1}}^{n} k^{p} \nabla_{n} \ell_{p}\right) \delta_{c d r_{1} s_{1} r_{2} s_{2} \ldots r_{m} s_{m}}^{a b p_{1} q_{1} p_{2} q_{2} \ldots p_{m} q_{m}} \times\left\{D^{r_{1}} \Theta_{q_{1}}^{s_{1}}+\left(k^{q} \nabla_{j} \ell_{q}\right) q^{j r_{1}} \Theta_{q_{1}}^{s_{1}}\right\} \\
& \times \sum_{t=0}^{m-1}{ }^{m-1} C_{t} 4^{t}\left({ }^{(D-2)} R_{p_{2} q_{2}}^{r_{2} s_{2}} \ldots{ }^{(D-2)} R_{p_{m-t} q_{m-t}}^{r_{m-t} s_{m-t}}\right) \\
& \times\left(\Theta_{p_{m-t+1}}^{r_{m-t+1}} \Psi_{q_{m-t+1}}^{s_{m-t+1}} \ldots \Theta_{p_{m}}^{r_{m}} \Psi_{q_{m}}^{s_{m}}\right) \\
& +\ldots
\end{aligned}
$$

Here we have made use of Eq. (20), again, as well as Eq. (21), and thrown away some metric variations into the dots. The summation has appeared in the same way as in Eq. (56), with the difference that there are only $m-1$ factors here.

Finally, we shall tackle the first term of Eq. (38), which, after multiplying with $\left[2^{m+1} /(m+1)\right]$, is

$$
\begin{aligned}
& \frac{2^{m+1}}{m+1} Q_{m+1}^{(1)}\left[\ell_{c}\right] \equiv \frac{2^{m+1}}{m+1}\left(4 P_{c d(m+1)}^{a b} \ell_{a} \ell^{c} \delta \Psi_{b}^{d}\right) \\
& =4 \ell_{a} \ell^{c} \delta \Psi_{b}^{d} \delta_{c d r_{1} s_{1} \ldots r_{m} s_{m}}^{a b p_{1} q_{1} \ldots p_{m} q_{m}} R_{p_{1} q_{1}}^{r_{1} s_{1}} \ldots R_{p_{m} q_{m}}^{r_{m} s_{m}} \\
& =4 \ell_{a} \ell^{c} \delta \Psi_{b}^{d} \delta_{c d r_{1} s_{1} \ldots r_{m} s_{m}}^{a b p_{1} q_{1} \ldots p_{m} q_{m}}\left(q_{p_{1}}^{a_{1}} q_{q_{1}}^{b_{1}} q_{c_{1}}^{r_{1}} q_{d_{1}}^{s_{1}} R_{a_{1} b_{1}}^{c_{1} d_{1}}\right) \ldots\left(q_{p_{m}}^{a_{m}} q_{q_{m}}^{b_{m}} q_{c_{m}}^{r_{m}} q_{d_{m}}^{s_{m}} R_{a_{m} b_{m}}^{c_{m} d_{m}}\right) \\
& -16 m \ell_{a} \ell^{c} \delta \Psi_{b}^{d} \delta_{c d r_{1} s_{1} \ldots r_{m} s_{m}}^{a b p_{1} q_{1} \ldots p_{m} q_{m}} k_{p_{1}}\left(\ell^{a_{1}} q_{q_{1}}^{b_{1}} q_{c_{1}}^{r_{1}} q_{d_{1}}^{s_{1}} R_{a_{1} b_{1}}^{c_{1} d_{1}}\right) \\
& \times\left(q_{p_{2}}^{a_{2}} q_{q_{2}}^{b_{2}} q_{c_{2}}^{r_{2}} q_{d_{2}}^{s_{2}} R_{a_{2} b_{2}}^{c_{2} d_{2}}\right) \ldots\left(q_{p_{m}}^{a_{m}} q_{q_{m}}^{b_{m}} q_{c_{m}}^{r_{m}} q_{d_{m}}^{s_{m}} R_{a_{m} b_{m}}^{c_{m} d_{m}}\right) \\
& +32{ }^{m} C_{2} \ell_{a} \ell^{c} \delta \Psi_{b}^{d} \delta_{c d r_{1} s_{1} \ldots r_{m} s_{m}}^{a b p_{1} q_{1} \ldots p_{m}}\left(\ell^{a_{1}} q_{q_{1}}^{b_{1}} q_{c_{1}}^{r_{1}} q_{d_{1}}^{s_{1}} R_{a_{1} b_{1}}^{c_{1} d_{1}}\right) k_{p_{1}}\left(\ell_{c_{2}} q_{q_{2}}^{b_{2}} q_{p_{2}}^{a_{2}} q_{d_{2}}^{s_{2}} R_{a_{2} b_{2}}^{c_{2} d_{2}}\right) k^{r_{2}} \\
& \times\left(q_{p_{3}}^{a_{3}} q_{q_{3}}^{b_{3}} q_{c_{3}}^{r_{3}} q_{d_{3}}^{s_{3}} R_{a_{3} b_{3}}^{c_{3} d_{3}}\right) \ldots\left(q_{p_{m}}^{a_{m}} q_{q_{m}}^{b_{m}} q_{c_{m}}^{r_{m}} q_{d_{m}}^{s_{m}} R_{a_{m} b_{m}}^{c_{m} d_{m}}\right) \\
& +16 m \ell_{a} \ell^{c} \delta \Psi_{b}^{d} \delta_{c d r_{1} s_{1} \ldots r_{m} s_{m}}^{a b p_{1} q_{1} \ldots p_{m}}\left(\ell^{a_{1}} q_{q_{1}}^{b_{1}} \ell_{c_{1}} q_{d_{1}}^{s_{1}} R_{a_{1} b_{1}}^{c_{1} d_{1}}\right) k_{p_{1}} k^{r_{1}} \\
& \times\left(q_{p_{2}}^{a_{2}} q_{q_{2}}^{b_{2}} q_{c_{2}}^{r_{2}} q_{d_{2}}^{s_{2}} R_{a_{2} b_{2}}^{c_{2} d_{2}}\right) \ldots\left(q_{p_{m}}^{a_{m}} q_{q_{m}}^{b_{m}} q_{c_{m}}^{r_{m}} q_{d_{m}}^{s_{m}} R_{a_{m} b_{m}}^{c_{m} d_{m}}\right) \\
& +\ldots \text {, }
\end{aligned}
$$

where the dots at the end indicate that we have removed some metric variation terms. Here also, we have expressed the Kronecker delta in terms of the induced metric $q_{b}^{a}$ and the null vectors $\ell_{a}$ and $k_{a}$. In this case, we get four non-zero terms on expansion: (a) a term with all the indices of all the Riemann tensors contracted with the induced metric; (b) a term with one index of just one Riemann tensor contracted with the null normal $\ell_{a}$ and all other Riemann tensor indices contracted with the induced metric $q_{b}^{a}$; (c) a term with one index each of two Riemann tensors contracted with $\ell_{a}$ and the rest with $q_{b}^{a}$ and (d) a term with two indices of one Riemann tensor contracted with $\ell_{a}$, with the other indices contracted with $q_{b}^{a}$. The second term is obtained in a manner similar to the second term in Eq. (55). But here there is no $k_{a}$ contracted a priori with the lower or upper indices of the determinant tensor. Therefore, terms with factors of the form $k^{r}\left(\ell_{c} q_{q}^{b} q_{p}^{a} q_{d}^{s} R_{a b}^{c d}\right)$ survive in addition to terms of the form $k_{p}\left(\ell^{a} q_{q}^{b} q_{c}^{r} q_{d}^{s} R_{a b}^{c d}\right)$, where the open indices are contracted with the determinant tensor. But these two types of terms are equivalent up to metric variations, as can be shown by lowering all upper indices and raising all lower indices. In proving this equivalence, one would have to throw away some metric variations to the dots while raising and lowering indices in $\delta \Psi_{b}^{d}$ and make use of the fact that $\Psi_{b}^{d}$ is a symmetric tensor. So we obtain an additional factor of two for this term in comparison with Eq. (55), giving an overall factor of $4 m$ on summing all such equivalent terms. The third term arises out of the possibility of having one Riemann tensor factor contracted as $k^{r}\left(\ell_{c} q_{q}^{b} q_{p}^{a} q_{d}^{s} R_{a b}^{c d}\right)$ and another contracted as $k_{p}\left(\ell^{a} q_{q}^{b} q_{c}^{r} q_{d}^{s} R_{a b}^{c d}\right)$. Symmetries of the Riemann tensor ensure that all such terms are equivalent. There are ${ }^{m} C_{2}$ ways of choosing two out of the $m$ Riemann tensors. Once two are chosen, one of the two upper indices of the first Riemann tensor can be contracted with $\ell_{c}$ while one of the two 
upper indices of the other are contracted with $\ell^{a}$, giving four possibilities. Similarly, there are four possibilities using the lower indices of the first and the upper indices of the second. In total, thus, there are 8 terms arising out of any pair of Riemann tensors. Thus, the total number of such terms formed is $8{ }^{m} C_{2}=4 m(m-1)$. The last term has one Riemann tensor contracted in the form $\ell^{a} q_{q}^{b} \ell_{c} q_{d}^{s} R_{a b}^{c d} k_{p} k^{r}$. Again, all such terms are equivalent. There are $m$ Riemann tensor factors to choose from, with each Riemann tensor offering two lower indices as options to contract with $\ell^{a}$ and two upper indices as options for contracting with $\ell_{c}$, giving four combinations in total per Riemann tensor. Thus, we obtain an overall factor of $4 \mathrm{~m}$.

We shall now simplify the terms in Eq. (58) one by one. We start with the first term, which has all the indices of all the Riemann tensors contracted with $q_{b}^{a}$. Making use of Eq. (20) and noting that this term has the same structure we encountered in Eq. (54), we can immediately write

$$
\begin{aligned}
\frac{2^{m+1}}{m+1} Q_{m+1}^{(1, a)}\left[\ell_{c}\right] \equiv & 4 \ell_{a} \ell^{c} \delta \Psi_{b}^{d} \delta_{c d r_{1} s_{1} \ldots r_{m} s_{m}}^{a b p_{1} q_{1} \ldots p_{m} q_{m}}\left(q_{p_{1}}^{a_{1}} q_{q_{1}}^{b_{1}} q_{c_{1}}^{r_{1}} q_{d_{1}}^{s_{1}} R_{a_{1} b_{1}}^{c_{1} d_{1}}\right) \ldots\left(q_{p_{m}}^{a_{m}} q_{q_{m}}^{b_{m}} q_{c_{m}}^{r_{m}} q_{d_{m}}^{s_{m}} R_{a_{m} b_{m}}^{c_{m} d_{m}}\right) \\
= & 4 \ell_{a} \ell^{c} \delta \Psi_{b}^{d} \delta_{c d r_{1} s_{1} \ldots r_{m} s_{m}}^{a b p_{1} \ldots q_{m} q_{m}} \\
& \times \sum_{t=0}^{m}{ }^{m} C_{t} 4^{t}\left({ }^{(D-2)} R_{p_{1} q_{1}}^{r_{1} s_{1}} \ldots{ }^{(D-2)} R_{p_{m-t} q_{m-t}}^{r_{m-t} s_{m-t}}\right)\left(\Theta_{p_{m-t+1}}^{r_{m-t+1}} \Psi_{q_{m-t+1}}^{s_{m-t+1}} \ldots \Theta_{p_{m}}^{r_{m}} \Psi_{q_{m}}^{s_{m}}\right) .
\end{aligned}
$$

Next, we simplify the second term in Eq. (58):

$$
\begin{aligned}
\frac{2^{m+1}}{m+1} Q_{m+1}^{(1, b)}\left[\ell_{c}\right] \equiv & -16 m \ell_{a} \ell^{c} \delta \Psi_{b}^{d} \delta_{c d r_{1} s_{1} \ldots r_{m} s_{m}}^{a b p_{1} q_{p_{1}} \ldots p_{m} q_{m}}\left(\ell^{a_{1}} q_{q_{1}}^{b_{1}} q_{c_{1}}^{r_{1}} q_{d_{1}}^{s_{1}} R_{a_{1} b_{1}}^{c_{1} d_{1}}\right) \\
& \times\left(q_{p_{2}}^{a_{2}} q_{q_{2}}^{b_{2}} q_{c_{2}}^{r_{2}} q_{d_{2}}^{s_{2}} R_{a_{2} b_{2}}^{c_{2} d_{2}}\right) \ldots\left(q_{p_{m}}^{a_{m}} q_{q_{m}}^{b_{m}} q_{c_{m}}^{r_{m}} q_{d_{m}}^{s_{m}} R_{a_{m} b_{m}}^{c_{m} d_{m}}\right) \\
= & -16 m \ell_{a} \ell^{c} k^{d} \delta \Psi_{p_{1}}^{r_{1}} \delta_{c d r_{1} s_{1} \ldots r_{m} s_{m}}^{a b p_{1} q_{1} \ldots p_{m} q_{m}}\left(q_{p_{2}}^{a_{2}} q_{q_{2}}^{b_{2}} q_{c_{2}}^{r_{2}} q_{d_{2}}^{s_{2}} R_{a_{2} b_{2}}^{c_{2} d_{2}}\right) \ldots\left(q_{p_{m}}^{a_{m}} q_{q_{m}}^{b_{m}} q_{c_{m}}^{r_{m}} q_{d_{m}}^{s_{m}} R_{a_{m} b_{m}}^{c_{m} d_{m}}\right) \\
& \times\left(\ell_{c_{1}} q_{q_{1}}^{b_{1}} q_{b}^{a_{1}} q_{d_{1}}^{s_{1}} R_{a_{1} b_{1}}^{c_{1} d_{1}}\right) \\
= & 32 m \ell_{a} \ell^{c} k^{d} \delta \Psi_{p_{1}}^{r_{1}} \delta_{c d r_{1} s_{1} \ldots r_{m} s_{m}}^{a b p_{1} q_{1} \ldots p_{m} q_{m}}\left(D_{b} \Theta_{q_{1}}^{s_{1}}+q_{b}^{j} \Theta_{q_{1}}^{s_{1}} k^{p} \nabla_{j} \ell_{p}\right) \\
& \times \sum_{t=0}^{m-1}{ }^{m-1} C_{t} 4^{t}\left({ }^{(D-2)} R_{p_{2} q_{2}}^{r_{2} s_{2}} \ldots{ }^{(D-2)} R_{p_{m-t} q_{m-t}}^{r_{m-t} s_{m-t}}\right) \\
& \times\left(\Theta_{p_{m-t+1}}^{r_{m-t+1}} \Psi_{q_{m-t+1}}^{s_{m-t+1}} \ldots \Theta_{p_{m}}^{r_{m}} \Psi_{q_{m}}^{s_{m}}\right) \\
& +\ldots .
\end{aligned}
$$

To obtain the second line above, we have lowered all the upper indices and raised all the lower indices, while throwing away metric variations to the dots. We have also exchanged some indices using the symmetries of the determinant tensor. The last step was obtained making use of Eq. (20) and Eq. (21) and simplifying in a manner similar to Eq. (57). Using the same two equations, we can simplify the third term in Eq. (58) as well. We have

$$
\begin{aligned}
\frac{2^{m+1}}{m+1} Q_{m+1}^{(1, c)}\left[\ell_{c}\right] \equiv & 32^{m} C_{2} \ell_{a} \ell^{c} \delta \Psi_{b}^{d} \delta_{c d r_{1} s_{1} r_{2} s_{2} \ldots r_{m} s_{m}}^{a b p_{1}}\left(\ell^{a_{1}} q_{q_{1}}^{b_{1}} q_{c_{1}}^{r_{1}} q_{d_{1}}^{s_{1}} R_{a_{1} b_{1}}^{c_{1} d_{1}}\right) k_{p_{1}}\left(\ell_{c_{2}} q_{q_{2}}^{b_{2}} q_{p_{2}}^{a_{2}} q_{d_{2}}^{s_{2}} R_{a_{2} b_{2}}^{c_{2} d_{2}}\right) k^{r_{2}} \\
& \times\left(q_{p_{3}}^{a_{3}} q_{q_{3}}^{b_{3}} q_{c_{3}}^{r_{3}} q_{d_{3}}^{s_{3}} R_{a_{3} b_{3}}^{c_{3} d_{3}}\right) \ldots\left(q_{p_{m}}^{a_{m}} q_{q_{m}}^{b_{m}} q_{c_{m}}^{r_{m}} q_{d_{m}}^{s_{m}} R_{a_{m} b_{m}}^{c_{m} d_{m}}\right) \\
= & -16 m(m-1) \ell_{a} \ell^{c} k_{b} k^{d} \delta \Psi_{p_{1}}^{r_{1}} \delta_{c d r_{1} s_{1} r_{2} s_{2} \ldots r_{m} s_{m}}^{a b p_{2} p_{2} q_{2} \ldots p_{m} q_{m}}\left(\ell^{a_{1}} q_{q_{1}}^{b_{1}} q_{c_{1}}^{r_{2}} q_{d_{1}}^{s_{1}} R_{a_{1} b_{1}}^{c_{1} d_{1}}\right)\left(\ell_{c_{2}} q_{q_{2}}^{b_{2}} q_{p_{2}}^{a_{2}} q_{d_{2}}^{s_{2}} R_{a_{2} b_{2}}^{c_{2} d_{2}}\right) \\
& \times\left(q_{p_{3}}^{a_{3}} q_{q_{3}}^{b_{3}} q_{c_{3}}^{r_{3}} q_{d_{3}}^{s_{3}} R_{a_{3} b_{3}}^{c_{3} d_{3}}\right) \ldots\left(q_{p_{m}}^{a_{m}} q_{q_{m}}^{b_{m}} q_{c_{m}}^{r_{m}} q_{d_{m}}^{s_{m}} R_{a_{m} b_{m}}^{c_{m} d_{m}}\right) \\
= & -64 m(m-1) \ell_{a} \ell^{c} k_{b} k^{d} \delta \Psi_{p_{1}}^{r_{1}} \delta_{c r_{1} p_{1} q_{1} r_{2} s_{2} \ldots r_{m} s_{m}}^{a b q_{2} p_{m}}\left[D^{r_{2}} \Theta_{q_{1}}^{s_{1}}+\left(k^{p} \nabla_{j} \ell_{p}\right) q^{j r_{2}} \Theta_{q_{1}}^{s_{1}}\right] \\
& \times\left[D_{p_{2}} \Theta_{q_{2}}^{s_{2}}+\left(k^{q} \nabla_{i} \ell_{q}\right) q_{p_{2}}^{i} \Theta_{q_{2}}^{s_{2}}\right]
\end{aligned}
$$




$$
\times \sum_{t=0}^{m-2}{ }^{m-2} C_{t} 4^{t}\left({ }^{(D-2)} R_{p_{3} q_{3}}^{r_{3} s_{3}} \ldots{ }^{(D-2)} R_{p_{m-t} q_{m-t}}^{r_{m-t} s_{m-t}}\right)\left(\Theta_{p_{m-t+1}}^{r_{m-t+1}} \Psi_{q_{m-t+1}}^{s_{m-t+1}} \ldots \Theta_{p_{m}}^{r_{m}} \Psi_{q_{m}}^{s_{m}}\right)
$$

We have used the symmetries of the determinant tensor to exchange some indices in the second step and have expanded the product as a sum in the last step as before.

Finally, we turn to the last term in Eq. (58). This can be simplified in the following manner:

$$
\begin{aligned}
\frac{2^{m+1}}{m+1} Q_{m+1}^{(1, d)}\left[\ell_{c}\right] \equiv & 16 m \ell_{a} \ell^{c} \delta \Psi_{b}^{d} \delta_{c d r_{1} s_{1} \ldots r_{m} s_{m}}^{a b p_{1} q_{1} \ldots p_{m} q_{m}}\left(\ell^{a_{1}} q_{q_{1}}^{b_{1}} \ell_{c_{1}} q_{d_{1}}^{s_{1}} R_{a_{1} b_{1}}^{c_{1} d_{1}}\right) k_{p_{1}} k^{r_{1}} \\
& \left(q_{p_{2}}^{a_{2}} q_{q_{2}}^{b_{2}} q_{c_{2}}^{r_{2}} q_{d_{2}}^{s_{2}} R_{a_{2} b_{2}}^{c_{2} d_{2}}\right) \ldots\left(q_{p_{m}}^{a_{m}} q_{q_{m}}^{b_{m}} q_{c_{m}}^{r_{m}} q_{d_{m}}^{s_{m}} R_{a_{m} b_{m}}^{c_{m} d_{m}}\right) \\
= & 16 m \ell_{a} \ell^{c} k_{b} k^{d} \delta \Psi_{p_{1}}^{r_{1}} \delta_{c d r_{1} s_{1} \ldots r_{m} s_{m}}^{a b p_{p_{1}} \ldots p_{m}}\left(-q_{i}^{s_{1}} q_{q_{1}}^{j} £_{\ell} \Theta_{j}^{i}+\kappa \Theta_{q_{1}}^{s_{1}}-\Theta_{i}^{s_{1}} \Theta_{q_{1}}^{i}\right) \\
& \times \sum_{t=0}^{m-1}{ }^{m-1} C_{t} 4^{t}\left({ }^{(D-2)} R_{p_{2} q_{2}}^{r_{2} s_{2}} \ldots{ }^{(D-2)} R_{p_{m-t} q_{m-t}}^{r_{m}-t}\right)\left(\Theta_{p_{m-t+1}}^{s_{m-t+1}} \Psi_{q_{m-t+1}}^{s_{m-t+1}} \ldots \Theta_{p_{m}}^{r_{m}} \Psi_{q_{m}}^{s_{m}}\right)
\end{aligned}
$$

which was obtained using Eq. (20) and Eq. (22). This completes our job of writing all the relevant terms in the boundary variation by decomposing them using the projections of the Riemann tensor near the null surface given in Eq. (20), Eq. (21) and Eq. (22). We will now regroup these terms appropriately.

\subsubsection{Grouping terms to obtain the total variation terms}

Thus, having written down the appropriate decomposition of all the terms, our next step would be to separate out the total variation terms while throwing away metric variations and their surface derivatives. For this purpose, it is advantageous to group certain terms together. First, note that the only terms involving the non-affinity parameter $\kappa$, which involves the derivatives of the metric along $k^{a}$, are in Eq. (54) and Eq. (62). In Eq. (54), $\kappa$ appears as a variation, while in Eq. (62) it appears outside the variation. Grouping these two terms together results in the following expression:

$$
\begin{aligned}
& \frac{2^{m+1}}{m+1} Q_{m+1}^{(3)}\left[\ell_{c}\right]+\frac{2^{m+1}}{m+1} Q_{m+1}^{(1, d)}\left[\ell_{c}\right] \\
& =4 \delta \kappa \ell_{a} \ell^{c} k_{b} k^{d} \delta_{c d r_{1} s_{1} \ldots r_{m} s_{m}}^{a b p_{1} q_{1} \ldots p_{m} q_{m}} \\
& \times \sum_{t=0}^{m}{ }^{m} C_{t} 4^{t}\left({ }^{(D-2)} R_{p_{1} q_{1}}^{r_{1} s_{1}} \ldots{ }^{(D-2)} R_{p_{m-t} q_{m-t}}^{r_{m-t} s_{m-t}}\right)\left(\Theta_{p_{m-t+1}}^{r_{m-t+1}} \Psi_{q_{m-t+1}}^{s_{m-t+1}} \ldots \Theta_{p_{m}}^{r_{m}} \Psi_{q_{m}}^{s_{m}}\right) \\
& +16 m \ell_{a} \ell^{c} k_{b} k^{d} \delta \Psi_{p_{1}}^{r_{1}} \delta_{c d r_{1} s_{1} \ldots r_{m} s_{m}}^{a b p_{1} q_{1} \ldots p_{m} q_{m}}\left(-q_{i}^{s_{1}} q_{q_{1}}^{j} £_{\ell} \Theta_{j}^{i}+\kappa \Theta_{q_{1}}^{s_{1}}-\Theta_{i}^{s_{1}} \Theta_{q_{1}}^{i}\right) \\
& \times \sum_{t=0}^{m-1}{ }^{m-1} C_{t} 4^{t}\left({ }^{(D-2)} R_{p_{2} q_{2}}^{r_{2} s_{2}} \ldots{ }^{(D-2)} R_{p_{m-t} q_{m-t}}^{r_{m-t} s_{m-t}}\right)\left(\Theta_{p_{m-t+1}}^{r_{m-t+1}} \Psi_{q_{m-t+1}}^{s_{m-t+1}} \ldots \Theta_{p_{m}}^{r_{m}} \Psi_{q_{m}}^{s_{m}}\right) \\
& =4 \delta \kappa \ell_{a} \ell^{c} k_{b} k^{d} \delta_{c d r_{1} s_{1} \ldots r_{m} s_{m}}^{a b p_{1} q_{1} \ldots p_{m} q_{m}} \\
& \times \sum_{t=0}^{m}{ }^{m} C_{t} 4^{t}\left({ }^{(D-2)} R_{p_{1} q_{1}}^{r_{1} s_{1}} \ldots{ }^{(D-2)} R_{p_{m-t} q_{m-t}}^{r_{m-t} s_{m-t}}\right)\left(\Theta_{p_{m-t+1}}^{r_{m-t+1}} \Psi_{q_{m-t+1}}^{s_{m-t+1}} \ldots \Theta_{p_{m}}^{r_{m}} \Psi_{q_{m}}^{s_{m}}\right) \\
& +4 \kappa \ell_{a} \ell^{c} k_{b} k^{d} \delta_{c d r_{1} s_{1} \ldots r_{m} s_{m}}^{a b p_{1} q_{1} \ldots p_{m} q_{m}} \times \sum_{t=0}^{m-1} m^{m-1} C_{t} 4^{t+1}\left({ }^{(D-2)} R_{p_{2} q_{2}}^{r_{2} s_{2}} \ldots{ }^{(D-2)} R_{p_{m-t} q_{m-t}}^{r_{m-t} s_{m-t}}\right) \\
& \times\left(\Theta_{q_{1}}^{s_{1}} \delta \Psi_{p_{1}}^{r_{1}}\right)\left(\Theta_{p_{m-t+1}}^{r_{m-t+1}} \Psi_{q_{m-t+1}}^{s_{m-t+1}} \ldots \Theta_{p_{m}}^{r_{m}} \Psi_{q_{m}}^{s_{m}}\right) \\
& +4 \ell_{a} \ell^{c} k_{b} k^{d} \delta \Psi_{p_{1}}^{r_{1}} \delta_{c d r_{1} s_{1} \ldots r_{m} s_{m}}^{a b p_{1} q_{1} \ldots p_{m} q_{m}}\left(-q_{i}^{s_{1}} q_{q_{1}}^{j} £_{\ell} \Theta_{j}^{i}-\Theta_{i}^{s_{1}} \Theta_{q_{1}}^{i}\right)
\end{aligned}
$$




$$
\begin{aligned}
& \times \sum_{t=0}^{m-1} m^{m-1} C_{t} 4^{t+1}\left({ }^{(D-2)} R_{p_{2} q_{2}}^{r_{2} s_{2}} \ldots{ }^{(D-2)} R_{p_{m-t} r_{m-t}}^{r_{m-t} s_{m-t}}\right)\left(\Theta_{p_{m-t+1}}^{r_{m-t+1}} \Psi_{q_{m-t+1}}^{s_{m-t+1}} \ldots \Theta_{p_{m}}^{r_{m}} \Psi_{q_{m}}^{s_{m}}\right) \\
&=\delta\left[4 \kappa \ell_{a} \ell^{c} k_{b} k^{d} \delta_{c d r_{1} s_{1} \ldots r_{m} s_{m}}^{a b p_{1} q_{1} \ldots p_{m} q_{m}} \sum_{t=0}^{m}{ }^{m} C_{t} 4^{t}\left({ }^{(D-2)} R_{p_{1} q_{1}}^{r_{1} s_{1}} \ldots{ }^{(D-2)} R_{p_{m-t} q_{m-t}}^{r_{m-t} s_{m-t}}\right)\right. \\
&\left.\times\left(\Theta_{p_{m-t+1}}^{r_{m-t+1}} \Psi_{q_{m-t+1}}^{s_{m-t+1}} \ldots \Theta_{p_{m}}^{r_{m}} \Psi_{q_{m}}^{s_{m}}\right)\right] \\
&-\delta\left[4 \ell_{a} \ell^{c} k_{b} k^{d} \delta_{c d r_{1} s_{1} \ldots r_{m} s_{m}}^{a b p_{1} q_{1} \ldots p_{m} q_{m}}\left(q_{i}^{s_{1}} q_{q_{1}}^{j} £_{\ell} \Theta_{j}^{i}+\Theta_{i}^{s_{1}} \Theta_{q_{1}}^{i}\right)\right. \\
& \quad \times \sum_{t=0}^{\left.{ }^{m-1}{ }^{m} C_{t+1} 4^{t+1}\left({ }^{(D-2)} R_{p_{2} q_{2}}^{r_{2} s_{2}} \ldots{ }^{(D-2)} R_{p_{m-t} q_{m-t}}^{r_{m-t} s_{m-t}}\right)\left(\Theta_{p_{m-t+1}}^{r_{m-t+1}} \Psi_{q_{m-t+1}}^{s_{m-t+1}} \ldots \Theta_{p_{m}}^{r_{m}} \Psi_{q_{m}}^{s_{m}}\right) \Psi_{p_{1}}^{r_{1}}\right]} \\
&+\ldots,
\end{aligned}
$$

where we have separated out the total variation term by removing the metric variations and variations of the surface derivatives of the metric to the dots, using the facts that only $\kappa$ and $\Psi_{b}^{a}$ among the tensors present above have derivatives of the metric along $k^{a}$ and that a $\delta$ on any $\Psi_{b}^{a}$ is equivalent to a $\delta$ on any other $\Psi_{b}^{a}$ due to the symmetries of the determinant tensor. Also,

$$
\begin{aligned}
& \delta_{c d r_{1} s_{1} \ldots r_{m} s_{m}}^{a b p_{1} q_{1} \ldots p_{m} q_{m}} \delta\left[\sum_{t=0}^{m}{ }^{m} C_{t} 4^{t}\left({ }^{(D-2)} R_{p_{1} q_{1}}^{r_{1} s_{1}} \ldots{ }^{(D-2)} R_{p_{m-t} r_{m-t}}^{r_{m-t} s_{m-t}}\right)\left(\Theta_{p_{m-t+1}}^{r_{m-t+1}} \Psi_{q_{m-t+1}}^{s_{m-t+1}} \ldots \Theta_{p_{m}}^{r_{m}} \Psi_{q_{m}}^{s_{m}}\right)\right] \\
& =\delta_{c d r_{1} s_{1} \ldots r_{m} s_{m}}^{a b p_{1} q_{1} \ldots p_{m} q_{m}} \delta\left[\sum_{t=1}^{m}{ }^{m} C_{t} 4^{t}\left({ }^{(D-2)} R_{p_{1} q_{1}}^{r_{1} s_{1}} \ldots{ }^{(D-2)} R_{p_{m-t} r_{m-t}}^{r_{m-t} s_{m-t}}\right)\left(\Theta_{p_{m-t+1}}^{r_{m-t+1}} \Psi_{q_{m-t+1}}^{s_{m-t+1}} \ldots \Theta_{p_{m}}^{r_{m}} \Psi_{q_{m}}^{s_{m}}\right)\right] \\
& +\ldots \\
& =\delta_{c d r_{1} s_{1} \ldots r_{m} s_{m}}^{a b p_{1} q_{1} \ldots p_{m} q_{m}}\left[\sum_{t=1}^{m}{ }^{m} C_{t} 4^{t}\left({ }^{(D-2)} R_{p_{1} q_{1}}^{r_{1} s_{1}} \ldots{ }^{(D-2)} R_{p_{m-t}-t q_{m-t}}^{r_{m-t} s_{m-t}}\right) \delta\left(\Theta_{p_{m-t+1}}^{r_{m-t+1}} \Psi_{q_{m-t+1}}^{s_{m-t+1}} \ldots \Theta_{p_{m}}^{r_{m}} \Psi_{q_{m}}^{s_{m}}\right)\right] \\
& +\ldots \\
& =\delta_{c d r_{1} s_{1} \ldots r_{m} s_{m}}^{a b p_{1} q_{1} \ldots p_{m} q_{m}}\left[\sum_{t=1}^{m}{ }^{m} C_{t} 4^{t} t\left({ }^{(D-2)} R_{p_{1} q_{1}}^{r_{1} s_{1}} \ldots{ }^{(D-2)} R_{p_{m-t}-t q_{m-t}}^{r_{m-t} s_{m-t}}\right)\left(\Theta_{p_{m-t+1}}^{r_{m-t+1}} \Psi_{q_{m-t+1}}^{s_{m-t+1}} \ldots \Theta_{p_{m}}^{r_{m}} \delta \Psi_{q_{m}}^{s_{m}}\right)\right] \\
& +\ldots \\
& =\delta_{c d r_{1} s_{1} \ldots r_{m} s_{m}}^{a b p_{1} q_{1} \ldots p_{m} q_{m}}\left[\sum_{t^{\prime}=0}^{m-1}{ }^{m} C_{t^{\prime}+1} 4^{t^{\prime}+1}\left(t^{\prime}+1\right)\left({ }^{(D-2)} R_{p_{1} q_{1}}^{r_{1} s_{1}} \ldots{ }^{(D-2)} R_{p_{m-t^{\prime}-1} q_{m-t^{\prime}-1}}^{r_{m-t^{\prime}} s_{m-t^{\prime}-1}}\right)\right. \\
& \left.\times\left(\Theta_{p_{m-t^{\prime}}}^{r_{m-t^{\prime}}} \Psi_{q_{m-t^{\prime}}}^{s_{m-t^{\prime}}} \ldots \Theta_{p_{m}}^{r_{m}} \delta \Psi_{q_{m}}^{s_{m}}\right)\right] \\
& +\ldots \\
& =\delta_{c d r_{1} s_{1} \ldots r_{m} s_{m}}^{a b p_{1} q_{1} \ldots p_{m} q_{m}}\left[\sum_{t^{\prime}=0}^{m-1} m^{m-1} C_{t^{\prime}} 4^{t^{\prime}+1}\left(\Theta_{q_{1}}^{s_{1}} \delta \Psi_{p_{1}}^{r_{1}}\right)\left({ }^{(D-2)} R_{p_{2} q_{2}}^{r_{2} s_{2}} \ldots{ }^{(D-2)} R_{p_{m-t^{\prime}} q_{m-t^{\prime}}}^{r_{m-t^{\prime}} s_{m-t^{\prime}}}\right)\right. \\
& \left.\times\left(\Theta_{p_{m-t^{\prime}+1}}^{r_{m-t^{\prime}+1}} \Psi_{q_{m-t^{\prime}+1}}^{s_{m-t^{\prime}+1}} \ldots \Theta_{p_{m}}^{r_{m}} \Psi_{q_{m}}^{s_{m}}\right)\right] \\
& +\ldots \text {, }
\end{aligned}
$$

which is how the $\delta \Psi_{p_{1}}^{r_{1}}$ term appears from the first term in Eq. (63).

Progressing forward, let us consider the term presented in Eq. (59). One can convert this term to a total variation in a straightforward manner as follows:

$$
\frac{2^{m+1}}{m+1} Q_{m+1}^{(1, a)}\left[\ell_{c}\right]=4 \ell_{a} \ell^{c} \delta \Psi_{b}^{d} \delta_{c d r_{1} s_{1} \ldots r_{m} s_{m}}^{a b p_{1} q_{1} \ldots p_{m} q_{m}}
$$




$$
\begin{aligned}
& \times \sum_{t=0}^{m}{ }^{m} C_{t} 4^{t}\left({ }^{(D-2)} R_{p_{1} q_{1}}^{r_{1} s_{1}} \ldots{ }^{(D-2)} R_{p_{m-t} q_{m-t}}^{r_{m-t} s_{m-t}}\right)\left(\Theta_{p_{m-t+1}}^{r_{m-t+1}} \Psi_{q_{m-t+1}}^{s_{m-t+1}} \ldots \Theta_{p_{m}}^{r_{m}} \Psi_{q_{m}}^{s_{m}}\right) \\
= & \delta\left[4 \ell_{a} \ell^{c} \Psi_{b}^{d} \delta_{c d r_{1} s_{1} \ldots r_{m} s_{m}}^{a b p_{1} q_{1} \ldots p_{m} q_{m}}\right. \\
& \left.\times \sum_{t=0}^{m} 4^{t} \frac{{ }^{m} C_{t}}{t+1}\left({ }^{(D-2)} R_{p_{1} q_{1}}^{r_{1} s_{1}} \ldots{ }^{(D-2)} R_{p_{m-t} q_{m-t}}^{r_{m-t} s_{m-t}}\right)\left(\Theta_{p_{m-t+1}}^{r_{m-t+1}} \Psi_{q_{m-t+1}}^{s_{m-t+1}} \ldots \Theta_{p_{m}}^{r_{m}} \Psi_{q_{m}}^{s_{m}}\right)\right] \\
& +\ldots
\end{aligned}
$$

where we have removed several terms involving variations of the metric and its surface derivatives into the dots. However, it turns out that the above variation is in fact zero. This is because if we expand the antisymmetric determinant tensor, the null normals $\ell_{a}$ and $\ell_{c}$ will be contracted either among themselves or with the other tensors present. All such contractions result in zero and thus we will receive a vanishing contribution from the above expression to the structure of the null boundary term.

For our next set of simplifications, we shall group the variations in Eq. (56) and Eq. (60) together.

This leads to

$$
\begin{aligned}
& \frac{2^{m+1}}{m+1} Q_{m+1}^{(2, a)}\left[\ell_{c}\right]+\frac{2^{m+1}}{m+1} Q_{m+1}^{(1, b)}\left[\ell_{c}\right] \\
& =8 \ell_{a} \ell^{c} k^{d} q_{b}^{j} \delta\left(k^{p} \nabla_{j} \ell_{p}\right) \delta_{c d r_{1} s_{1} \ldots r_{m} s_{m}}^{a b p_{1} q_{1} \ldots p_{m} q_{m}} \\
& \times \sum_{t=0}^{m}{ }^{m} C_{t} 4^{t}\left({ }^{(D-2)} R_{p_{1} q_{1}}^{r_{1} s_{1}} \ldots{ }^{(D-2)} R_{p_{m-t} q_{m-t}}^{r_{m-t} s_{m-t}}\right)\left(\Theta_{p_{m-t+1}}^{r_{m-t+1}} \Psi_{q_{m-t+1}}^{s_{m-t+1}} \ldots \Theta_{p_{m}}^{r_{m}} \Psi_{q_{m}}^{s_{m}}\right) \\
& +32 m \ell_{a} \ell^{c} k^{d} \delta \Psi_{p_{1}}^{r_{1}} \delta_{c d r_{1} s_{1} \ldots r_{m} s_{m}}^{a b p_{1} q_{1} \ldots p_{m} q_{m}}\left(D_{b} \Theta_{q_{1}}^{s_{1}}+q_{b}^{j} \Theta_{q_{1}}^{s_{1}} k^{p} \nabla_{j} \ell_{p}\right) \\
& \times \sum_{t=0}^{m-1}{ }^{m-1} C_{t} 4^{t}\left({ }^{(D-2)} R_{p_{2} q_{2}}^{r_{2} s_{2}} \ldots{ }^{(D-2)} R_{p_{m-t} q_{m-t}}^{r_{m-t} s_{m-t}}\right) \\
& \times\left(\Theta_{p_{m-t+1}}^{r_{m-t+1}} \Psi_{q_{m-t+1}}^{s_{m-t+1}} \ldots \Theta_{p_{m}}^{r_{m}} \Psi_{q_{m}}^{s_{m}}\right) \\
& =\delta\left[8 \ell_{a} \ell^{c} k^{d} q_{b}^{j}\left(k^{p} \nabla_{j} \ell_{p}\right) \delta_{c d r_{1} s_{1} \ldots r_{m} s_{m}}^{a b p_{1} q_{1} \ldots p_{m} q_{m}}\right. \\
& \left.\times \sum_{t=0}^{m}{ }^{m} C_{t} 4^{t}\left({ }^{(D-2)} R_{p_{1} q_{1}}^{r_{1} s_{1}} \ldots{ }^{(D-2)} R_{p_{m-t} q_{m-t}}^{r_{m-t} s_{m-t}}\right)\left(\Theta_{p_{m-t+1}}^{r_{m-t+1}} \Psi_{q_{m-t+1}}^{s_{m-t+1}} \ldots \Theta_{p_{m}}^{r_{m}} \Psi_{q_{m}}^{s_{m}}\right)\right] \\
& +\delta\left[8 \ell_{a} \ell^{c} k^{d}\left(D_{b} \Theta_{q_{1}}^{s_{1}}\right) \delta_{c d r_{1} s_{1} \ldots r_{m} s_{m}}^{a b p_{1} q_{1} \ldots p_{m} q_{m}}\right. \\
& \left.\times \sum_{t=0}^{m-1}{ }^{m} C_{t+1} 4^{t+1}\left({ }^{(D-2)} R_{p_{2} q_{2}}^{r_{2} s_{2}} \ldots{ }^{(D-2)} R_{p_{m-t} q_{m-t}}^{r_{m-t} s_{m-t}}\right) \times\left(\Theta_{p_{m-t+1}}^{r_{m-t+1}} \Psi_{q_{m-t+1}}^{s_{m-t+1}} \ldots \Theta_{p_{m}}^{r_{m}} \Psi_{q_{m}}^{s_{m}} \Psi_{p_{1}}^{r_{1}}\right)\right] \\
& +\ldots \text {, }
\end{aligned}
$$

where the dots again stand for terms with only variations of the metric and its surface derivatives. Here too, the derivatives along $k^{a}$ are present in only two of the tensors involved, $k^{p} \nabla_{a} \ell_{p}$ and $\Psi_{b}^{a}$ being the tensors this time around.

The final grouping that we need to do is of the terms in Eq. (57) and Eq. (61). These terms are also dependent on $k^{p} \nabla_{a} \ell_{p}$, but in a quadratic manner. The simplification in this case involves a little more work, but the steps are analogous to those of our previous simplifications. The terms are

$$
\frac{2^{m+1}}{m+1} Q_{m+1}^{(2, b)}\left[\ell_{c}\right]+\frac{2^{m+1}}{m+1} Q_{m+1}^{(1, c)}\left[\ell_{c}\right]
$$




$$
\begin{aligned}
& =-32 m \ell_{a} \ell^{c} k_{b} k^{d} \delta\left(q_{p_{1}}^{i} k^{p} \nabla_{i} \ell_{p}\right) \delta_{c d r_{1} s_{1} r_{2} s_{2} \ldots r_{m} s_{m}}^{a b b p_{1} q_{1} q_{2} q_{2} p_{m} q_{m}}\left\{D^{r_{1}} \Theta_{q_{1}}^{s_{1}}+\left(k^{q} \nabla_{j} \ell_{q}\right) q^{j r_{1}} \Theta_{q_{1}}^{s_{1}}\right\} \\
& \times \sum_{t=0}^{m-1}{ }^{m-1} C_{t} 4^{t}\left({ }^{(D-2)} R_{p_{2} q_{2}}^{r_{2} s_{2}} \ldots{ }^{(D-2)} R_{p_{m-t} r_{m-t}}^{r_{m-t} s_{m-t}}\right)\left(\Theta_{p_{m-t+1}}^{r_{m-t+1}} \Psi_{q_{m-t+1}}^{s_{m-t+1}} \ldots \Theta_{p_{m}}^{r_{m}} \Psi_{q_{m}}^{s_{m}}\right) \\
& -64 m(m-1) \ell_{a} \ell^{c} k_{b} k^{d} \delta \Psi_{p_{1}}^{r_{1}} \delta_{c d r_{1} s_{1} r_{2} s_{2} \ldots r_{m} s_{m}}^{a b p_{1} q_{1} p_{2} q_{2}}\left\{D^{r_{2}} \Theta_{q_{1}}^{s_{1}}+\left(k^{p} \nabla_{i} \ell_{p}\right) q^{i r_{2}} \Theta_{q_{1}}^{s_{1}}\right\} \\
& \times\left\{D_{p_{2}} \Theta_{q_{2}}^{s_{2}}+\left(k^{q} \nabla_{j} \ell_{q}\right) q_{p_{2}}^{j} \Theta_{q_{2}}^{s_{2}}\right\} \\
& \times \sum_{t=0}^{m-2}{ }^{m-2} C_{t} 4^{t}\left({ }^{(D-2)} R_{p_{3} q_{3}}^{r_{3} s_{3}} \ldots{ }^{(D-2)} R_{p_{m-t} q_{m-t}}^{r_{m-t} s_{m-t}}\right) \times\left(\Theta_{p_{m-t+1}}^{r_{m-t+1}} \Psi_{q_{m-t+1}}^{s_{m-t+1}} \ldots \Theta_{p_{m}}^{r_{m}} \Psi_{q_{m}}^{s_{m}}\right) \\
& =-32 m \ell_{a} \ell^{c} k_{b} k^{d} \delta_{c d r_{1} s_{1} r_{2} s_{2} \ldots r_{m} s_{m}}^{a b p_{1} q_{1} p_{2} q_{2} \ldots p_{m} q_{m}}\left[\left(D^{r_{1}} \Theta_{q_{1}}^{s_{1}}\right) \delta\left(q_{p_{1}}^{i} k^{p} \nabla_{i} \ell_{p}\right)+\Theta_{q_{1}}^{s_{1}} q^{j r_{1}}\left(k^{q} \nabla_{j} \ell_{q}\right) \delta\left(q_{p_{1}}^{i} k^{p} \nabla_{i} \ell_{p}\right)\right] \\
& \times \sum_{t=0}^{m-1}{ }^{m-1} C_{t} 4^{t}\left({ }^{(D-2)} R_{p_{2} q_{2}}^{r_{2} s_{2}} \ldots{ }^{(D-2)} R_{p_{m-t} r_{m-t}}^{r_{m-t} s_{m-t}}\right)\left(\Theta_{p_{m-t+1}}^{r_{m-t+1}} \Psi_{q_{m-t+1}}^{s_{m-t+1}} \ldots \Theta_{p_{m}}^{r_{m}} \Psi_{q_{m}}^{s_{m}}\right) \\
& -64 m(m-1) \ell_{a} \ell^{c} k_{b} k^{d} \delta_{c d r_{1} s_{1} r_{2} s_{2} \ldots r_{m} s_{m}}^{a b p_{1} q_{1} p_{2} q_{2} \ldots p_{m} q_{m}} \\
& \times\left[\left(D^{r_{2}} \Theta_{q_{1}}^{s_{1}}\right)\left(D_{p_{2}} \Theta_{q_{2}}^{s_{2}}\right)+\left(k^{p} \nabla_{i} \ell_{p}\right) q^{i r_{2}} \Theta_{q_{1}}^{s_{1}}\left(k^{q} \nabla_{j} \ell_{q}\right) q_{p_{2}}^{j} \Theta_{q_{2}}^{s_{2}}+2\left(D^{r_{2}} \Theta_{q_{1}}^{s_{1}}\right)\left(k^{q} \nabla_{j} \ell_{q}\right) q_{p_{2}}^{j} \Theta_{q_{2}}^{s_{2}}\right] \delta \Psi_{p_{1}}^{r_{1}} \\
& \times \sum_{t=0}^{m-2}{ }^{m-2} C_{t} 4^{t}\left({ }^{(D-2)} R_{p_{3} q_{3}}^{r_{3} s_{3}} \ldots{ }^{(D-2)} R_{p_{m-t} q_{m-t}}^{r_{m-t} s_{m-t}}\right) \times\left(\Theta_{p_{m-t+1}}^{r_{m-t+1}} \Psi_{q_{m-t+1}}^{s_{m-t+1}} \ldots \Theta_{p_{m}}^{r_{m}} \Psi_{q_{m}}^{s_{m}}\right) \\
& =-\delta\left[4 m \ell_{a} \ell^{c} k_{b} k^{d} \delta_{c d r_{1} s_{1} r_{2} s_{2} \ldots r_{m} s_{m}}^{a b p_{1} q_{1} p_{2} q_{2} \ldots p_{m} q_{m}}\left(q_{p_{1}}^{i} k^{p} \nabla_{i} \ell_{p}\right)\left(q^{j r_{1}} k^{q} \nabla_{j} \ell_{q}\right) \Theta_{q_{1}}^{s_{1}}\right. \\
& \left.\times \sum_{t=0}^{m-1}{ }^{m-1} C_{t} 4^{t+1}\left({ }^{(D-2)} R_{p_{2} q_{2}}^{r_{2} s_{2}} \ldots{ }^{(D-2)} R_{p_{m-t} q_{m-t}}^{r_{m-t} s_{m-t}}\right) \times\left(\Theta_{p_{m-t+1}}^{r_{m-t+1}} \Psi_{q_{m-t+1}}^{s_{m-t+1}} \ldots \Theta_{p_{m}}^{r_{m}} \Psi_{q_{m}}^{s_{m}}\right)\right] \\
& -\delta\left[4 m \ell_{a} \ell^{c} k_{b} k^{d} \delta_{c d r_{1} s_{1} r_{2} s_{2} \ldots r_{m} s_{m}}^{a b p_{1} q_{1} p_{2} q_{2} \ldots p_{m} q_{m}}\left(D_{p_{2}} \Theta_{q_{2}}^{s_{2}}\right)\left(D^{r_{2}} \Theta_{q_{1}}^{s_{1}}\right)\right. \\
& \times \sum_{t=0}^{m-2}{ }^{m-1} C_{t+1} 4^{t+2}\left({ }^{(D-2)} R_{p_{3} q_{3}}^{r_{3} s_{3}} \ldots{ }^{(D-2)} R_{p_{m-t} q_{m-t}}^{r_{m-t} s_{m-t}}\right) \\
& \left.\times\left(\Theta_{p_{m-t+1}}^{r_{m-t+1}} \Psi_{q_{m-t+1}}^{s_{m-t+1}} \ldots \Theta_{p_{m}}^{r_{m}} \Psi_{q_{m}}^{s_{m}} \Psi_{p_{1}}^{r_{1}}\right)\right] \\
& -\delta\left[8 m \ell_{a} \ell^{c} k_{b} k^{d} \delta_{c d r_{1} s_{1} r_{2} s_{2} \ldots r_{m} s_{m}}^{a b p_{1} q_{1} p_{2} q_{2} \ldots p_{m} q_{m}}\left(q_{p_{1}}^{j} k^{p} \nabla_{j} \ell_{p}\right)\left(D^{r_{1}} \Theta_{q_{1}}^{s_{1}}\right)\right. \\
& \left.\times \sum_{t=0}^{m-1}{ }^{m-1} C_{t} 4^{t+1}\left({ }^{(D-2)} R_{p_{2} q_{2}}^{r_{2} s_{2}} \ldots{ }^{(D-2)} R_{p_{m-t} r_{m-t}}^{r_{m-t} s_{m-t}}\right) \times\left(\Theta_{p_{m-t+1}}^{r_{m-t+1}} \Psi_{q_{m-t+1}}^{s_{m-t+1}} \ldots \Theta_{p_{m}}^{r_{m}} \Psi_{q_{m}}^{s_{m}}\right)\right] \\
& +\ldots \text {, }
\end{aligned}
$$

where we have again thrown away metric variations and their surface derivatives. In the second step, we have expanded out the products and used the result that the two cross terms arising in the product in the $\delta \Psi_{p_{1}}^{r_{1}}$ term, i.e., the terms with $D^{r_{2}} \Theta_{q_{1}}^{s_{1}}\left(k^{q} \nabla_{j} \ell_{q}\right) q_{p_{2}}^{j} \Theta_{q_{2}}^{s_{2}}$ and $D_{p_{2}} \Theta_{q_{2}}^{s_{2}}\left(k^{p} \nabla_{i} \ell_{p}\right) q^{i r_{2}} \Theta_{q_{1}}^{s_{1}}$, are equivalent up to metric variations. This equivalence can be seen by using the symmetry properties of the determinant tensor first to exchange the indices on the $\Theta s$ and then raising and lowering all indices present, while throwing away metric variations, to match the positions of the indices $p_{2}$ and $r_{2}$. As one can see, there are five different terms that occur after the expansion of the products. These are the terms containing the following expressions: i) $\left(D^{r_{1}} \Theta_{q_{1}}^{s_{1}}\right) \delta\left(q_{p_{1}}^{i} k^{p} \nabla_{i} \ell_{p}\right)$, ii) $\Theta_{q_{1}}^{s_{1}} q^{j r_{1}}\left(k^{q} \nabla_{j} \ell_{q}\right) \delta\left(q_{p_{1}}^{i} k^{p} \nabla_{i} \ell_{p}\right)$, iii) $\left(D^{r_{2}} \Theta_{q_{1}}^{s_{1}}\right)\left(D_{p_{2}} \Theta_{q_{2}}^{s_{2}}\right) \delta \Psi_{p_{1}}^{r_{1}}$, iv $)\left(k^{p} \nabla_{i} \ell_{p}\right) q^{i r_{2}} \Theta_{q_{1}}^{s_{1}}\left(k^{q} \nabla_{j} \ell_{q}\right) q_{p_{2}}^{j} \Theta_{q_{2}}^{s_{2}} \delta \Psi_{p_{1}}^{r_{1}}$ and finally v) $\left(D^{r_{2}} \Theta_{q_{1}}^{s_{1}}\right)\left(k^{q} \nabla_{j} \ell_{q}\right) q_{p_{2}}^{j} \Theta_{q_{2}}^{s_{2}} \delta \Psi_{p_{1}}^{r_{1}}$.

Let us examine how these terms arise from the variations given in the last step of Eq. (67). The easiest variation to tackle in Eq. (67) is the second one. Since $\left(D_{p_{2}} \Theta_{q_{2}}^{s_{2}}\right)\left(D^{r_{2}} \Theta_{q_{1}}^{s_{1}}\right)$ does not contain 
any derivatives along $k^{a}$, the only normal derivatives present in this term are in the $\Psi \mathrm{s}$. There are $(t+1) \Psi$ s present, all of them being equivalent due to the symmetries of the determinant tensor. Thus, we obtain the term iii) in our list above, the one containing $\left(D^{r_{2}} \Theta_{q_{1}}^{s_{1}}\right)\left(D_{p_{2}} \Theta_{q_{2}}^{s_{2}}\right) \delta \Psi_{p_{1}}^{r_{1}}$, from this variation. Next, we consider the last variation term in Eq. (67). Here, the derivatives along $k^{a}$ are present in $k^{p} \nabla_{j} \ell_{p}$ and the $\Psi_{\mathrm{s}}$. The variation acting on $k^{p} \nabla_{j} \ell_{p}$ gives us the term i) in our list above, while variation acting on the $\Psi_{\mathrm{s}}$ produces the term $\left.\mathrm{v}\right)$ in the list. We are left with the terms ii) and iv) in our list. These come from the first variation in Eq. (67), where again the derivatives along $k^{a}$ are present in $k^{p} \nabla_{j} \ell_{p}$, now present twice, and the $\Psi_{\mathrm{s}}$. Thus, we have verified that the variation terms in Eq. (67) correctly provide all the terms we started with.

\subsubsection{Obtaining the boundary term}

We have obtained all the results that we require to write down an explicit expression for the null boundary term. Putting together Eq. (63), Eq. (65), Eq. (66) and Eq. (67) (where Eq. (65) does not make any contribution to the boundary term, as we have stated before), and adding the factor of $\sqrt{q}$ back (which can be taken inside the $\delta$ at the cost of metric variation terms), we obtain the total structure of the boundary variation for Lanczos-Lovelock gravity as

$$
\begin{aligned}
& \frac{2^{m+1}}{m+1} \sqrt{q} Q_{m+1}\left[\ell_{c}\right] \\
& \equiv \frac{2^{m+1}}{m+1}\left(\sqrt{q} Q_{m+1}^{(1)}\left[\ell_{c}\right]+\sqrt{q} Q_{m+1}^{(2)}\left[\ell_{c}\right]+\sqrt{q} Q_{m+1}^{(3)}\left[\ell_{c}\right]\right) \\
& =-\delta\left[8 \sqrt{q} m \ell_{a} \ell^{c} k_{b} k^{d} \delta_{c d r_{1} s_{1} r_{2} s_{2} \ldots r_{m} s_{m}}^{a b p_{1} q_{1} p_{2} q_{2} \ldots p_{m} q_{m}}\left(q_{p_{1}}^{j} k^{p} \nabla_{j} \ell_{p}\right)\left(D^{r_{1}} \Theta_{q_{1}}^{s_{1}}\right)\right.
\end{aligned}
$$

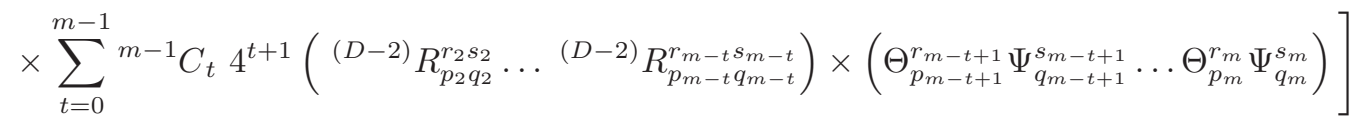

$$
\begin{aligned}
& -\delta\left[4 \sqrt{q} m \ell_{a} \ell^{c} k_{b} k^{d} \delta_{c d r_{1} s_{1} r_{2} s_{2} \ldots r_{m} s_{m}}^{a b p_{m} q_{1} p_{2} q_{2} \ldots p_{m} q_{m}}\left(D_{p_{2}} \Theta_{q_{2}}^{s_{2}}\right)\left(D^{r_{2}} \Theta_{q_{1}}^{s_{1}}\right)\right. \\
& \times \sum_{t=0}^{m-2}{ }^{m-1} C_{t+1} 4^{t+2}\left({ }^{(D-2)} R_{p_{3} q_{3}}^{r_{3} s_{3}} \ldots{ }^{(D-2)} R_{p_{m-t} q_{m-t}}^{r_{m-t} s_{m-t}}\right) \\
& \left.\times\left(\Theta_{p_{m-t+1}}^{r_{m-t+1}} \Psi_{q_{m-t+1}}^{s_{m-t+1}} \ldots \Theta_{p_{m}}^{r_{m}} \Psi_{q_{m}}^{s_{m}} \Psi_{p_{1}}^{r_{1}}\right)\right] \\
& -\delta\left[4 \sqrt{q} m \ell_{a} \ell^{c} k_{b} k^{d} \delta_{c d r_{1} s_{1} r_{2} s_{2} \ldots r_{m} s_{m}}^{a b p_{1} q_{1} p_{2} q_{2} \ldots p_{m} q_{m}}\left(q_{p_{1}}^{i} k^{p} \nabla_{i} \ell_{p}\right)\left(q^{j r_{1}} k^{q} \nabla_{j} \ell_{q}\right) \Theta_{q_{1}}^{s_{1}}\right. \\
& \left.\times \sum_{t=0}^{m-1}{ }^{m-1} C_{t} 4^{t+1}\left({ }^{(D-2)} R_{p_{2} q_{2}}^{r_{2} s_{2}} \ldots{ }^{(D-2)} R_{p_{m-t} q_{m-t}}^{r_{m-t} s_{m-t}}\right) \times\left(\Theta_{p_{m-t+1}}^{r_{m-t+1}} \Psi_{q_{m-t+1}}^{s_{m-t+1}} \ldots \Theta_{p_{m}}^{r_{m}} \Psi_{q_{m}}^{s_{m}}\right)\right] \\
& +\delta\left[8 \sqrt{q} \ell_{a} \ell^{c} k^{d}\left(D_{b} \Theta_{q_{1}}^{s_{1}}\right) \delta_{c d r_{1} s_{1} \ldots r_{m} s_{m}}^{a b p_{1} q_{1} \ldots p_{m}}\right. \\
& \left.\times \sum_{t=0}^{m-1}{ }^{m} C_{t+1} 4^{t+1}\left({ }^{(D-2)} R_{p_{2} q_{2}}^{r_{2} s_{2}} \ldots{ }^{(D-2)} R_{p_{m-t} q_{m-t}}^{r_{m-t} s_{m-t}}\right) \times\left(\Theta_{p_{m-t+1}}^{r_{m-t+1}} \Psi_{q_{m-t+1}}^{s_{m-t+1}} \ldots \Theta_{p_{m}}^{r_{m}} \Psi_{q_{m}}^{s_{m}} \Psi_{p_{1}}^{r_{1}}\right)\right] \\
& +\delta\left[8 \sqrt{q} \ell_{a} \ell^{c} k^{d} q_{b}^{j}\left(k^{p} \nabla_{j} \ell_{p}\right) \delta_{c d r_{1} s_{1} \ldots r_{m} s_{m}}^{a b p_{1} q_{1} \ldots p_{m} q_{m}}\right. \\
& \left.\times \sum_{t=0}^{m}{ }^{m} C_{t} 4^{t}\left({ }^{(D-2)} R_{p_{1} q_{1}}^{r_{1} s_{1}} \ldots{ }^{(D-2)} R_{p_{m-t} q_{m-t}}^{r_{m-t} s_{m-t}}\right)\left(\Theta_{p_{m-t+1}}^{r_{m-t+1}} \Psi_{q_{m-t+1}}^{s_{m-t+1}} \ldots \Theta_{p_{m}}^{r_{m}} \Psi_{q_{m}}^{s_{m}}\right)\right]
\end{aligned}
$$




$$
\begin{aligned}
& -\delta\left[4 \sqrt{q} \ell_{a} \ell^{c} k_{b} k^{d} \delta_{c d r_{1} s_{1} \ldots r_{m} s_{m}}^{a b p_{1} q_{1} \ldots p_{m} q_{m}}\left(q_{i}^{s_{1}} q_{q_{1}}^{j} £_{\ell} \Theta_{j}^{i}+\Theta_{i}^{s_{1}} \Theta_{q_{1}}^{i}\right)\right. \\
& \left.\quad \times \sum_{t=0}^{m-1}{ }^{m} C_{t+1} 4^{t+1}\left({ }^{(D-2)} R_{p_{2} q_{2}}^{r_{2} s_{2}} \ldots{ }^{(D-2)} R_{p_{m-t} q_{m-t}}^{r_{m-t} s_{m-t}}\right)\left(\Theta_{p_{m-t+1}}^{r_{m-t+1}} \Psi_{q_{m-t+1}}^{s_{m}-t+1} \ldots \Theta_{p_{m}}^{r_{m}} \Psi_{q_{m}}^{s_{m}}\right) \Psi_{p_{1}}^{r_{1}}\right] \\
& +\delta\left[4 \sqrt{q} \kappa \ell_{a} \ell^{c} k_{b} k^{d} \delta_{c d r_{1} s_{1} \ldots r_{m} s_{m}}^{a b p_{1} q_{1} \ldots p_{m} q_{m}} \sum_{t=0}^{m}{ }^{m} C_{t} 4^{t}\left({ }^{(D-2)} R_{p_{1} q_{1}}^{r_{1} s_{1}} \ldots{ }^{(D-2)} R_{p_{m-t} q_{m-t}}^{r_{m}-s_{m-t}}\right)\right. \\
& \left.\times\left(\Theta_{p_{m-t+1}}^{r_{m-t+1}} \Psi_{q_{m-t+1}}^{s_{m-t+1}} \ldots \Theta_{p_{m}}^{r_{m}} \Psi_{q_{m}}^{s_{m}}\right)\right]+\ldots,
\end{aligned}
$$

where all the terms which do not have variations of the derivatives of the metric along $k^{a}$ have been represented by the dots. From the above decomposition, we can straight away identify the boundary term for $m^{\text {th }}$ order Lanczos-Lovelock gravity [the above decomposition is for $(m+1)^{\text {th }}$ order] that will cancel the variations of non-tangential derivatives of the metric (derivatives in directions not tangent to the boundary) appearing in the boundary variation of the action.

Grouping terms and arranging tensors according to the order of indices of the determinant tensor, we can write the expression for the null boundary term for $m^{\text {th }}$ order Lanczos-Lovelock gravity as

$$
\begin{aligned}
& \sqrt{q} \mathcal{B}_{\mathrm{LL}}^{(m)}\left[\ell_{c}\right] \\
& =4 \sqrt{q} \frac{m}{2^{m}} \delta_{c d r_{1} s_{1} r_{2} s_{2} \ldots r_{m-1} s_{m-1}}^{a b p_{1} q_{1} p_{2} q_{2} \ldots p_{m-1} q_{m-1}} \ell_{a} k^{d} \\
& \times\left\{-\left[2\left(q_{b}^{i} k^{p} \nabla_{i} \ell_{p}\right)+\kappa k_{b}\right]\right. \\
& \sum_{t=0}^{m-1}{ }^{m-1} C_{t} 4^{t}\left({ }^{(D-2)} R_{p_{1} q_{1}}^{r_{1} s_{1}} \ldots{ }^{(D-2)} R_{p_{m-t-1} q_{m-t-1}}^{r_{m-t-1} s_{m-t-1}}\right)\left(\Theta_{p_{m-t}}^{r_{m-t}} \Psi_{q_{m-t}}^{s_{m-t}} \ldots \Theta_{p_{m-1}}^{r_{m-1}} \Psi_{q_{m-1}}^{s_{m-1}}\right) \\
& +\sum_{t=0}^{m-2} 4^{t+1}\left\{(m-1) k_{b}\left(q_{p_{1}}^{i} k^{p} \nabla_{i} \ell_{p}\right)\left[\left(q^{j r_{1}} k^{q} \nabla_{j} \ell_{q}\right) \Theta_{q_{1}}^{s_{1}}+2 D^{r_{1}} \Theta_{q_{1}}^{s_{1}}\right]^{m-2} C_{t}\right. \\
& \left.+\Psi_{p_{1}}^{r_{1}}\left[k_{b}\left(q_{i}^{s_{1}} q_{q_{1}}^{j} £_{\ell} \Theta_{j}^{i}+\Theta_{i}^{s_{1}} \Theta_{q_{1}}^{i}\right)-2 D_{b} \Theta_{q_{1}}^{s_{1}}\right]^{m-1} C_{t+1}\right\}
\end{aligned}
$$

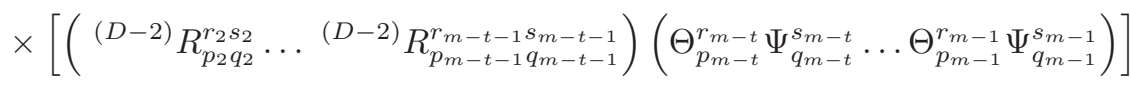

$$
\begin{aligned}
& +(m-1) k_{b} \Psi_{p_{1}}^{r_{1}}\left(D^{r_{2}} \Theta_{q_{1}}^{s_{1}}\right)\left(D_{p_{2}} \Theta_{q_{2}}^{s_{2}}\right) \\
& \left.\sum_{t=0}^{m-3}{ }^{m-2} C_{t+1} 4^{t+2}\left({ }^{(D-2)} R_{p_{3} q_{3}}^{r_{3} s_{3}} \ldots{ }^{(D-2)} R_{p_{m-t-1} r_{m-t-1} s_{m-t-1}}^{r_{m-t-1}}\right)\left(\Theta_{p_{m-t} r_{m-t}}^{r_{m-t}} \Psi_{q_{m-t}}^{s_{m-t}} \ldots \Theta_{p_{m-1}}^{r_{m-1}} \Psi_{q_{m-1}}^{s_{m-1}}\right)\right\} .
\end{aligned}
$$

We can simplify this result further. Let us examine the determinant tensor present:

$$
\begin{aligned}
& \delta_{c d r_{1} s_{1} r_{2} s_{2} \ldots r_{m-1} s_{m-1}}^{a b p_{1} q_{1} p_{2} q_{2} \ldots p_{m-1} q_{m-1}}=-\delta_{d c r_{1} s_{1} r_{2} s_{2} \ldots r_{m-1} s_{m-1}}^{a b p_{1} q_{1} p_{2} q_{2} \ldots p_{m-1} q_{m-1}}
\end{aligned}
$$

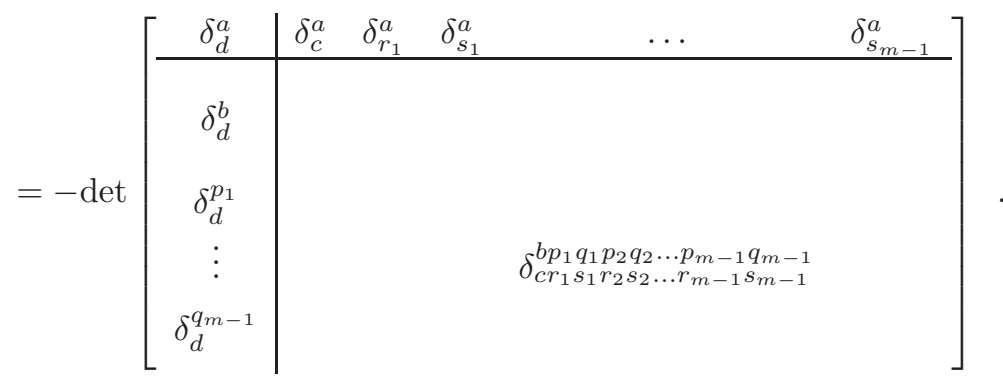


Expanding the determinant along the first row, we can see that only the contribution from $\delta_{d}^{a}$ survives in Eq. (69), since all the other delta functions in the first row contract $\ell_{a}$ with such tensors as to give zero. Thus, effectively,

$$
\begin{aligned}
& \delta_{c d r_{1} s_{1} r_{2} s_{2} \ldots r_{m-1} s_{m-1}}^{a b p_{1} q_{1} p_{2} q_{2} \ldots p_{m-1} q_{m-1}} \rightarrow-\delta_{d}^{a} \delta_{c r_{1} s_{1} r_{2} s_{2} \ldots p_{m-1} p_{m-1} q_{1} p_{2} q_{m-1} \ldots p_{m-1} q_{m-1}}^{b}
\end{aligned}
$$

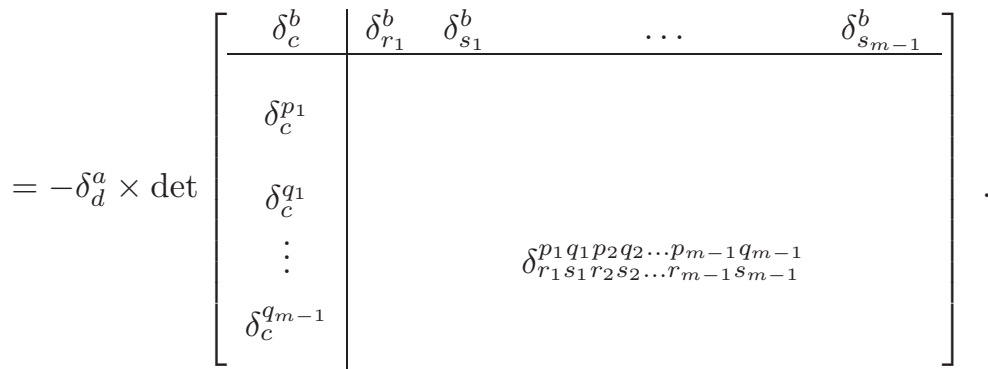

By making a similar argument but by expanding along the first column and looking at contractions of $\ell_{c}$, we can reduce this determinant further down to give

$$
\delta_{c d r_{1} s_{1} r_{2} s_{2} \ldots r_{m-1} s_{m-1}}^{a b p_{1} q_{1} p_{2} q_{2} \ldots p_{m-1} q_{m-1}} \rightarrow-\delta_{d}^{a} \delta_{c}^{b} \delta_{r_{1} s_{1} r_{2} s_{2} \ldots r_{m-1} s_{m-1}}^{p_{1} q_{1} p_{2} q_{2} \ldots p_{m-1} q_{m-1}}
$$

Substituting this back in Eq. (69), we obtain

$$
\begin{aligned}
& \sqrt{q} \mathcal{B}_{\mathrm{LL}}^{(m)}\left[\ell_{c}\right] \\
& =4 \sqrt{q} \frac{m}{2^{m}} \delta_{r_{1} s_{1} r_{2} s_{2} \ldots r_{m-1} s_{m-1}}^{p_{1} q_{1} p_{2} q_{2} \ldots p_{m-1} q_{m-1}} \\
& \times\left\{\kappa \sum_{t=0}^{m-1}{ }^{m-1} C_{t} 4^{t}\left({ }^{(D-2)} R_{p_{1} q_{1}}^{r_{1} s_{1}} \ldots{ }^{(D-2)} R_{p_{m-t-1} r_{m-t-1}}^{r_{m-t-1} s_{m-t-1}}\right)\left(\Theta_{p_{m-t}}^{r_{m-t}} \Psi_{q_{m-t}}^{s_{m-t}} \ldots \Theta_{p_{m-1}}^{r_{m-1}} \Psi_{q_{m-1}}^{s_{m-1}}\right)\right. \\
& -\sum_{t=0}^{m-2} 4^{t+1}\left\{(m-1)\left(q_{p_{1}}^{i} k^{p} \nabla_{i} \ell_{p}\right)\left[\left(q^{j r_{1}} k^{q} \nabla_{j} \ell_{q}\right) \Theta_{q_{1}}^{s_{1}}+2 D^{r_{1}} \Theta_{q_{1}}^{s_{1}}\right]^{m-2} C_{t}\right. \\
& \left.+\Psi_{p_{1}}^{r_{1}}\left[\left(q_{i}^{s_{1}} q_{q_{1}}^{j} £_{\ell} \Theta_{j}^{i}+\Theta_{i}^{s_{1}} \Theta_{q_{1}}^{i}\right)\right]^{m-1} C_{t+1}\right\} \\
& \times\left[\left({ }^{(D-2)} R_{p_{2} q_{2}}^{r_{2} s_{2}} \ldots{ }^{(D-2)} R_{p_{m-t-1} q_{m-t-1}}^{r_{m-t-1} s_{m-t-1}}\right)\left(\Theta_{p_{m-t}^{r_{m-t}}}^{r_{m-t}} \Psi_{q_{m-t}}^{s_{m-t}} \ldots \Theta_{p_{m-1}}^{r_{m-1}} \Psi_{q_{m-1}}^{s_{m-1}}\right)\right] \\
& -(m-1) \Psi_{p_{1}}^{r_{1}}\left(D^{r_{2}} \Theta_{q_{1}}^{s_{1}}\right)\left(D_{p_{2}} \Theta_{q_{2}}^{s_{2}}\right) \\
& \left.\sum_{t=0}^{m-3}{ }^{m-2} C_{t+1} 4^{t+2}\left({ }^{(D-2)} R_{p_{3} q_{3}}^{r_{3} s_{3}} \ldots{ }^{(D-2)} R_{p_{m-t-1} r_{m-t-1}}^{r_{m-t-1} s_{m-t-1}}\right)\left(\Theta_{p_{m-t} r_{m-t}}^{r_{m-t}} \Psi_{q_{m-t}}^{s_{m-t}} \ldots \Theta_{p_{m-1}}^{r_{m-1}} \Psi_{q_{m-1}}^{s_{m-1}}\right)\right\} .
\end{aligned}
$$

The object $-q_{j}^{i} k^{p} \nabla_{i} \ell_{p}$ appearing in these boundary terms is in fact the Hájiček 1-form $\Omega_{j}[66-68]$. The expression for the null boundary term as presented in Eq. (73) is for $m^{\text {th }}$ order LanczosLovelock gravity, but one can write down the boundary term for general Lanczos-Lovelock gravity by simply incorporating a summation over the Lanczos-Lovelock order $m$ with appropriate coefficients $c_{m}$. Thus, just by considering the variation of the Lanczos-Lovelock gravitational action on a null boundary, we have been able to determine the relevant null boundary term for LanczosLovelock gravity.

Note that we have not commented on the normal being future-directed or past-directed. This is because the normal is not a priori future-directed or past-directed in our conventions. In the conventions of [15], where the null normal is defined to be future-directed, the boundary term expression will pick up an overall negative sign. This is explained in detail in Appendix E.

In [69], the late time growth of complexity for charged black holes in Lanczos-Lovelock gravity was calculated with the assumption that the null boundary terms are polynomials in the intrinsic and extrinsic geometric quantities of the null boundary. It was shown that such polynomial boundary terms do not contribute to the action at late times. Now that we have derived the complete 
expression for the boundary term, we can check whether their assumption is valid. The intrinsic and extrinsic geometric quantities of the null boundary that they used were taken from [70]. Comparing with our notation, we see that the correspondence is as follows: $\hat{\mathcal{R}}_{C D}^{A B}$ corresponds to our ${ }^{(D-2)} R_{c d}^{a b}, \mathcal{B}_{A B}$ to $\Theta_{a b}, \mathcal{K}_{a b}$ to $\Psi_{a b}, \omega_{A}$ to the Hájiček 1-form $\Omega_{a}$ and $\kappa$ to our $\kappa$, up to overall signs and factors. But our boundary term does not appear to be completely polynomial in these objects, since it also contains derivatives of $\Theta_{a b}$. Hence, we are not sure if the conclusions in [69] are still valid. This matter requires further exploration.

To check the correctness of this result, we shall evaluate this expression for the values of $m=1$ and $m=2$, which are the cases of Einstein-Hilbert gravity and Gauss-Bonnet gravity, respectively. (The case of $m=0$ gives zero, which is expected since the cosmological constant term does not need a boundary term.)

\subsection{Obtaining Einstein-Hilbert and Gauss-Bonnet null boundary terms from the general Lanczos-Lovelock result}

In this section, we shall consider two specific cases of the general result in Eq. (73): one being the case of Einstein-Hilbert action, for which the null boundary term is available in the literature $[11,12,15]$; and the other being Gauss-Bonnet gravity, for which we have determined the null boundary term in Section 4.2 .

\subsection{1 $m=1$ Einstein-Hilbert case}

Consider substituting $m=1 \mathrm{in} \mathrm{Eq.} \mathrm{(73).} \mathrm{Of} \mathrm{the} \mathrm{three} \mathrm{sums} \mathrm{present} \mathrm{in} \mathrm{the} \mathrm{equation,} \mathrm{only} \mathrm{the} \mathrm{first}$ one will survive. The last sum can be summarily dropped because of the $(m-1)$ factor. The second sum runs over positive integer values of $t$ from 0 to $m-2$, which in this case would be 0 to -1 . This is to be interpreted as the sum not being able to run at all, giving a zero contribution. The first sum runs from 0 to $m-1$, and thus will have only the single $t=0$ term in the present case. The determinant tensor has the indices $p_{i}, q_{i}, r_{i}$ and $s_{i}$ with $i$ running from 1 to $m-1$, which is 1 to 0 for $m=1$. This means that the indices $p_{i}, q_{i}, r_{i}$ and $s_{i}$ will not be present, the determinant tensor in Eq. (73) will not be present and all the ${ }^{(D-2)} R_{p_{i} q_{i}}^{r_{i} s_{i}}$ and $\Theta_{p_{i}}^{r_{i}} \Psi_{q_{i}}^{s_{i}}$ will not be present. This does not mean that the determinant tensor has to be replaced by zero. Rather, we should replace it with unity. This is clearer if we look at the previous form in Eq. (69), where the determinant tensor would reduce from $\delta_{c d r_{1} s_{1} r_{2} s_{2} \ldots r_{m-1} s_{m-1}}^{a b p_{m} q_{2} p_{2} q_{2} \ldots p_{m-1} q_{m-1}}$ to just $\delta_{c d}^{a b}$. Further, ${ }^{m-1} C_{t}={ }^{0} C_{0}=1$, using the usual formula for ${ }^{n} C_{k}$ with $0 !=1$. Thus, the $t=0$ term reads

$$
\sqrt{q} \mathcal{B}_{\mathrm{LL}}^{(1)}\left[\ell_{c}\right]=2 \sqrt{q} \kappa
$$

which matches with the expression for the null boundary term of the Einstein-Hilbert action available in the literature $[12,15]$. Our definitions and conventions follow [12]. The difference in choosing the direction of the null normal leads to the difference of a minus sign from [15], as explained in Appendix E. We have assumed $\ell_{a}=A \nabla_{a} \phi$ and assumed that all $\phi=$ constant surfaces are null surfaces. Thus, our result can be directly compared with the result in Appendix G in the arXiv version of [12], but after imposing the additional assumption of $\ell^{a} \ell_{a}=0$ everywhere. The expression for the boundary term provided there is $2(\sqrt{-g} / A)(\kappa+\Theta)$. From Appendix D, $(\sqrt{-g} / A)=\sqrt{q}$ and hence this reduces to $2 \sqrt{q}(\kappa+\Theta)$ in our case. The $2 \sqrt{q} \Theta$ term contains only derivatives of the metric along the null boundary, so it may be dropped, as has been done in [15], if our aim is just to cancel off derivatives along $k^{a}$ from the boundary variation. So we are left with just $2 \sqrt{q} \kappa$, which is our result above. [On the other hand, the unified boundary term in $[13,17]$ reduces to $2 \sqrt{q}(\kappa+\Theta)$ in the null case. The question of which of the boundary terms, the bare $2 \sqrt{q} \Theta$ or the whole $2 \sqrt{q}(\kappa+\Theta)$, is the correct boundary term requires further exploration. The derivation of the boundary term in this work has been performed with the aim of eliminating derivatives of the metric in directions off the null boundary only.] The additional assumption of $\ell^{a} \ell_{a}=0$ everywhere that we have assumed in this work just changes the expression for $\kappa$ from $\kappa=\ell^{a} \partial_{a} \ln A-\left(k^{a} / 2\right) \partial_{a}\left(\ell^{b} \ell_{b}\right)$ to $\kappa=\ell^{a} \partial_{a} \ln A$. 


\subsection{2 $m=2$ Gauss-Bonnet case}

For the case of Gauss-Bonnet gravity, we substitute $m=2$ in Eq. (73). The last sum in Eq. (73) does not contribute because the sum goes over the invalid range of 0 to -1 . The other two sums do contribute. The first sum goes over the two values $t=0$ and $t=1$ and the second sum just consists of the $t=0$ term. From the first sum, we obtain

$$
\begin{aligned}
\sqrt{q} \mathcal{B}_{\mathrm{LL}}^{(2,1)}\left[\ell_{c}\right] & =2 \sqrt{q} \delta_{r_{1} s_{1}}^{p_{1} q_{1}} \kappa\left({ }^{(D-2)} R_{p_{1} q_{1}}^{r_{1} s_{1}}+4 \Theta_{p_{1}}^{r_{1}} \Psi_{q_{1}}^{s_{1}}\right) \\
& =2 \sqrt{q} \kappa\left(\delta_{r_{1}}^{p_{1}} \delta_{s_{1}}^{q_{1}}-\delta_{s_{1}}^{p_{1}} \delta_{r_{1}}^{q_{1}}\right)\left({ }^{(D-2)} R_{p_{1} q_{1}}^{r_{1} s_{1}}+4 \Theta_{p_{1}}^{r_{1}} \Psi_{q_{1}}^{s_{1}}\right) \\
& =2 \sqrt{q} \kappa\left(2^{(D-2)} R+4 \Theta \Psi-4 \Theta_{a b} \Psi^{a b}\right) .
\end{aligned}
$$

Moving over to the second sum, we have

$$
\begin{aligned}
& \sqrt{q} \mathcal{B}_{\mathrm{LL}}^{(2,2)}\left[\ell_{c}\right]= 2 \sqrt{q} \delta_{r_{1} s_{1}}^{p_{1} q_{1}}\left\{-4\left(q_{p_{1}}^{i} k^{j} \nabla_{i} \ell_{j}\right)\left[\left(q^{k r_{1}} k^{q} \nabla_{k} \ell_{q}\right) \Theta_{q_{1}}^{s_{1}}+2 D^{r_{1}} \Theta_{q_{1}}^{s_{1}}\right]\right. \\
&\left.-4 \Psi_{p_{1}}^{r_{1}}\left(q_{i}^{s_{1}} q_{q_{1}}^{j} £_{\ell} \Theta_{j}^{i}+\Theta_{i}^{s_{1}} \Theta_{q_{1}}^{i}\right)\right\} \\
&=-8 \sqrt{q}\left(\delta_{r_{1}}^{p_{1}} \delta_{s_{1}}^{q_{1}}-\delta_{s_{1}}^{p_{1}} \delta_{r_{1}}^{q_{1}}\right)\left(q_{p_{1}}^{i} k^{j} \nabla_{i} \ell_{j}\right)\left[\left(q^{k r_{1}} k^{q} \nabla_{k} \ell_{q}\right) \Theta_{q_{1}}^{s_{1}}+2 D^{r_{1}} \Theta_{q_{1}}^{s_{1}}\right] \\
&-8 \sqrt{q}\left(\delta_{r_{1}}^{p_{1}} \delta_{s_{1}}^{q_{1}}-\delta_{s_{1}}^{p_{1}} \delta_{r_{1}}^{q_{1}}\right) \Psi_{p_{1}}^{r_{1}}\left(q_{i}^{s_{1}} q_{q_{1}}^{j} £_{\ell} \Theta_{j}^{i}+\Theta_{i}^{s_{1}} \Theta_{q_{1}}^{i}\right) \\
&=-8 \sqrt{q}\left[q^{k i}\left(k^{j} \nabla_{i} \ell_{j}\right)\left(k^{q} \nabla_{k} \ell_{q}\right) \Theta+2\left(k^{j} \nabla_{i} \ell_{j}\right) D^{i} \Theta\right. \\
&\left.-\left(k^{j} \nabla_{i} \ell_{j}\right)\left(k^{q} \nabla_{k} \ell_{q}\right) \Theta^{i k}-2\left(k^{j} \nabla_{i} \ell_{j}\right) D^{r} \Theta_{r}^{i}\right] \\
&-8 \sqrt{q}\left[\Psi\left(q_{i}^{j} £_{\ell} \Theta_{j}^{i}+\Theta_{i}^{j} \Theta_{j}^{i}\right)-\left(\Psi_{i}^{j} £_{\ell} \Theta_{j}^{i}+\Psi_{p}^{q} \Theta_{i}^{p} \Theta_{q}^{i}\right)\right] .
\end{aligned}
$$

In order to compare this with Eq. (52), we just need to make one more simplification. We have

$$
\begin{aligned}
q_{i}^{j} £_{\ell} \Theta_{j}^{i}=£_{\ell} \Theta-\Theta_{j}^{i} £_{\ell} q_{i}^{j} & =\ell^{a} \nabla_{a} \Theta-\Theta_{j}^{i}\left(\ell^{a} \nabla_{a} q_{i}^{j}+q_{a}^{j} \nabla_{i} \ell^{a}-q_{i}^{a} \nabla_{a} \ell^{j}\right) \\
& =\ell^{a} \nabla_{a} \Theta-\left[\Theta_{j}^{i} \ell^{a} \nabla_{a}\left(k^{j} \ell_{i}+\ell^{j} k_{i}\right)+\Theta_{j}^{i} \Theta_{i}^{j}-\Theta_{j}^{i} \Theta_{i}^{j}\right] \\
& =\ell^{a} \nabla_{a} \Theta=\frac{d \Theta}{d \lambda},
\end{aligned}
$$

where $\lambda$ was defined below Eq. (44). Using Eq. (77) and combining Eq. (75) and Eq. (76), the complete $m=2$ null boundary term is

$$
\begin{aligned}
\sqrt{q} \mathcal{B}_{\mathrm{LL}}^{(2)}\left[\ell_{c}\right]= & 2 \sqrt{q} \kappa\left(2^{(D-2)} R+4 \Theta \Psi-4 \Theta_{a b} \Psi^{a b}\right) \\
& -8 \sqrt{q}\left[q^{k i}\left(k^{j} \nabla_{i} \ell_{j}\right)\left(k^{q} \nabla_{k} \ell_{q}\right) \Theta+2\left(k^{j} \nabla_{i} \ell_{j}\right) D^{i} \Theta\right. \\
& \left.-\left(k^{j} \nabla_{i} \ell_{j}\right)\left(k^{q} \nabla_{k} \ell_{q}\right) \Theta^{i k}-2\left(k^{j} \nabla_{i} \ell_{j}\right) D^{r} \Theta_{r}^{i}\right] \\
& -8 \sqrt{q}\left[\Psi \frac{d \Theta}{d \lambda}+\Psi \Theta_{i}^{j} \Theta_{j}^{i}-\Psi_{i}^{j} £_{\ell} \Theta_{j}^{i}-\Psi_{p}^{q} \Theta_{i}^{p} \Theta_{q}^{i}\right]
\end{aligned}
$$

which can be seen to coincide with Eq. (52), the null boundary term for Gauss-Bonnet gravity we had derived earlier.

Thus, the null boundary term presented in Eq. (73) leads to the null boundary terms for both Einstein-Hilbert gravity and Gauss-Bonnet gravity for appropriate values of $m$.

\section{Conclusion}

Lanczos-Lovelock models of gravity, which includes general relativity, have actions that are not well-posed, and need boundary terms. Even though the boundary terms for Lanczos-Lovelock 
theories in the context of non-null surfaces have been known for some time, the null boundary terms were not available in the literature. In this work, we have filled this gap by providing appropriate null boundary terms. The framework and the assumptions that we have used are provided in Section 3. The following are the assumptions that we have imposed: i) $B=1$; ii) $\ell^{2}=0$ everywhere, i.e. the null boundary is part of a null foliation; iii) $\delta \ell^{2}=0$ and $\delta k^{2}=0$ everywhere. These assumptions have been made for simplifying the calculations. But we shall shortly argue that the results are not expected to change even if we remove these assumptions, enforcing the last two only on the null surface. We hope to show this explicitly in a future work.

We first derived a null boundary term for Gauss-Bonnet gravity in Section 4.2. This boundary term is the following integral over the null boundary:

$$
\begin{aligned}
\int_{\partial \mathcal{M}} d \psi d^{d-2} z \sqrt{q} \mathcal{B}_{\mathrm{GB}} & \\
=\int_{\partial \mathcal{M}} d \psi d^{d-2} z 4 \sqrt{q}[ & \kappa\left(\left({ }^{(D-2)} R-2 \Theta_{q}^{p} \Psi_{p}^{q}+2 \Theta \Psi\right)\right. \\
& +2 \Psi_{q}^{p} £_{\ell} \Theta_{p}^{q}-2 \Psi \ell^{a} \nabla_{a} \Theta+2 \Theta_{c}^{a} \Theta_{b}^{c} \Psi_{a}^{b}-2 \Psi \Theta_{b}^{a} \Theta_{a}^{b} \\
& \left.-4\left(D^{m} \Theta-D^{a} \Theta_{a}^{m}\right)\left(k^{q} \nabla_{m} \ell_{q}\right)-2\left(k^{q} \nabla_{m} \ell_{q}\right)\left(k^{p} \nabla_{a} \ell_{p}\right)\left(\Theta q^{m a}-\Theta^{m a}\right)\right],
\end{aligned}
$$

where $\psi$ is the scalar whose level surfaces give the auxiliary null foliation with $k_{a}=\nabla_{a} \psi, d^{d-2} z$ stands for $d z^{1} d z^{2} \ldots d z^{(D-2)}$ with the $z^{A}$ standing for the $D-2$ coordinates on the codimension-2 surfaces orthogonal to $k^{a}$ on the null boundary (see Appendix C and Appendix D). The definitions of the null surface objects involved are given in Section 3.

Then, in Section 4.3, we derived the null boundary term for $m^{\text {th }}$ order Lanczos-Lovelock as

$$
\begin{aligned}
& \int_{\partial \mathcal{M}} d \psi d^{d-2} z \sqrt{q} \mathcal{B}_{\mathrm{LL}}^{(m)}\left[\ell_{c}\right] \\
& =\int_{\partial \mathcal{M}} d \psi d^{d-2} z 4 \sqrt{q} \frac{m}{2^{m}} \delta_{r_{1} s_{1} r_{2} s_{2} \ldots r_{m-1} s_{m-1}}^{p_{1} q_{1} q_{2} q_{2}} \\
& \quad \times\left\{\kappa \sum_{t=0}^{m-1}{ }^{m-1} C_{t} 4^{t}\left({ }^{(D-2)} R_{p_{1} q_{1}}^{r_{1} s_{1}} \ldots{ }^{(D-2)} R_{p_{m-t-1} q_{m-t-1}}^{r_{m-t-1} s_{m-t-1}}\right)\left(\Theta_{p_{m-t}}^{r_{m-t}} \Psi_{q_{m-t}}^{s_{m-t}} \ldots \Theta_{p_{m-1}}^{r_{m-1}} \Psi_{q_{m-1}}^{s_{m-1}}\right)\right. \\
& \quad-\sum_{t=0}^{m-2} 4^{t+1}\left\{(m-1)\left(q_{p_{1}}^{i} k^{p} \nabla_{i} \ell_{p}\right)\left[\left(q^{j r_{1}} k^{q} \nabla_{j} \ell_{q}\right) \Theta_{q_{1}}^{s_{1}}+2 D^{r_{1}} \Theta_{q_{1}}^{s_{1}}\right]^{m-2} C_{t}\right. \\
& \left.\quad+\Psi_{p_{1}}^{r_{1}}\left[\left(q_{i}^{s_{1}} q_{q_{1}}^{j} £_{\ell} \Theta_{j}^{i}+\Theta_{i}^{s_{1}} \Theta_{q_{1}}^{i}\right)\right]^{m-1} C_{t+1}\right\} \\
& \quad \times\left[\left({ }^{(D-2)} R_{p_{2} q_{2}}^{r_{2} s_{2}} \ldots{ }^{(D-2)} R_{p_{m-t-1} q_{m-t-1}}^{r_{m-t-1} s_{m-t-1}}\right)\left(\Theta_{p_{m-t}}^{r_{m-t}} \Psi_{q_{m-t}-t}^{s_{m-t}} \ldots \Theta_{p_{m-1}}^{r_{m-1}} \Psi_{q_{m-1}}^{s_{m-1}}\right)\right] \\
& \quad-(m-1) \Psi_{p_{1}}^{r_{1}}\left(D^{r_{2}} \Theta_{q_{1}}^{s_{1}}\right)\left(D_{p_{2}} \Theta_{q_{2}}^{s_{2}}\right) \\
& \left.\quad \sum_{t=0}^{m-3}{ }^{m-2} C_{t+1} 4^{t+2}\left({ }^{(D-2)} R_{p_{3} q_{3}}^{r_{3} s_{3} \ldots}{ }^{(D-2)} R_{p_{m-t-1} q_{m-t-1}}^{r_{m-t-1} s_{m-t-1}}\right)\left(\Theta_{p_{m-t}}^{r_{m-t}} \Psi_{q_{m-t}}^{s_{m-t} \ldots} \Theta_{p_{m-1}}^{r_{m-1}} \Psi_{q_{m-1}-1}^{s_{m-1}}\right)\right\} .
\end{aligned}
$$

with $-q_{j}^{i} k^{p} \nabla_{i} \ell_{p}$ being the Hájiček 1-form $\Omega_{j}[66-68]$. We have checked that this boundary term reduces to the corresponding expressions for general relativity and Gauss-Bonnet in Section 4.4. These expressions are derived in the conventions specified in our earlier paper [12]. To convert to the conventions followed in [15], one just needs to append an overall minus sign to the boundary terms, as explained in Appendix E.

Let us briefly discuss whether the above results depend on the assumptions we had imposed. A review of the calculations suggests that lifting the assumption $B=1$ will only lead to the 
minor change of $\sqrt{q}$ being replaced by $B \sqrt{q}$ in the boundary terms. The remaining assumptions are $\ell^{2}=0, \delta \ell^{2}=0$ and $\delta k^{2}=0$ everywhere. But there are multiple reasons to expect that restricting these assumptions to be valid only on the null surface is enough to reproduce our results. Firstly, [15] reproduced the result in [12] for the Einstein-Hilbert null boundary term using a framework claimed to involve only tangential derivatives. We have followed [12] here, but we expect the framework used in [15] to reproduce our results, just as in the Einstein-Hilbert case. Then, the behaviour of $\ell^{2}, \delta \ell^{2}$ and $\delta k^{2}$ off the null surface should not matter. Secondly, our boundary terms contains all the intrinsic and extrinsic tensors associated with a null surface as given in [70]. Explicitly, $\gamma_{A B}$ corresponds to our $q_{a b}, \hat{\mathcal{R}}_{C D}^{A B}$ to our ${ }^{(D-2)} R_{c d}^{a b}, \mathcal{B}_{A B}$ to $\Theta_{a b}, \mathcal{K}_{a b}$ to $\Psi_{a b}, \omega_{A}$ to the Hájiček 1-form $\Omega_{a}$ and $\kappa$ to our $\kappa$, up to overall signs and factors. If these are indeed all the extrinsic and intrinsic tensors associated with a null surface, we do not expect any additional contribution to arise if restrict the assumptions of $\ell^{2}=0, \delta \ell^{2}=0$ and $\delta k^{2}=0$ to the null surface. Finally, we have done a rapid calculation of the Gauss-Bonnet boundary term lifting the assumption of $B=1$ and enforcing $\ell^{2}=0, \delta \ell^{2}=0$ and $\delta k^{2}=0$ only on the null surface. The result was in accordance with the above arguments. Thus, we have strong reasons to believe that our boundary terms are valid in a more general setting. We hope to present a careful calculation of the Gauss-Bonnet and Lanczos-Lovelock boundary terms to verify our claims above, along with a unified treatment of spacelike, timelike and null boundary terms for Lanczos-Lovelock gravity, elsewhere.

The derivation presented here opens up several directions of exploration. We have not kept track of the terms with variations of the metric or variations of the surface derivatives of the metric on the boundary, unlike in our earlier papers $[12,13,58]$. These terms can be partitioned into a Dirichlet variation term and a surface total derivative term. From the Dirichlet variation term, one can discover the degrees of freedom to be fixed on the null boundary for Lanczos-Lovelock models of gravity, the conjugate momenta corresponding to these degrees of freedom, the associated Poisson bracket structure and route for quantization. The surface total derivative term in the variational principle is also of importance, since the structure of corner terms associated with a null boundary can be derived from this term [15]. (One can also derive the corner terms from the boundary term by obtaining the corners as the limit of smooth portions of the boundary [22]. See [71] for a comparison of the three methods of finding corner terms, with the claim that they are equivalent.) These corner terms as well as the null boundary terms are important in checking the "complexity=action" conjecture for Lanczos-Lovelock theories (see [15] where the null boundary term for Einstein-Hilbert action was used to check the conjecture for general relativity). This is perhaps the one of most current interest among the possible applications of our results. In [59], the Lanczos-Lovelock corner terms for non-null boundaries were derived, and it was argued that these terms can be extended in a straightforward manner to the case of corners on null boundaries. Our derivation here provides us with the resources to check this claim explicitly. The claim was further used to calculate the late time growth of complexity for charged black holes in LanczosLovelock gravity in [69]. With the assumption that the null boundary terms are polynomials in extrinsic and intrinsic geometric quantities of the null surface, it is demonstrated that they do not contribute to the action. As our result explicitly demonstrates, there are also non-polynomial terms present in the null boundary terms for Lanczos-Lovelock gravity. Thus, it will be interesting to check whether the null boundary term contribution indeed vanishes at late times. Also, these results can presumably be extended to scenarios where the null boundary term is of importance, now that we have derived the appropriate null boundary terms. There are many other applications that we can imagine, and we hope the community puts our results to fruitful use.

\section{Acknowledgements}

Research of SC is funded by the INSPIRE Faculty Fellowship (Reg. No. DST/INSPIRE/04/2018/000893) from Department of Science and Technology, Government of India. Research of KP is supported by the SERB-NPDF grant (No. PDF/2017/002782), from DST, Government of India, the DGAPA postdoctoral fellowship from UNAM and the Fondecyt Postdoctoral fellowship (No. 3180421) 
from the Government of Chile. KP would like to thank Perimeter Institute for kind hospitality during a stay, discussions in which duration led to this project. SC thanks IIT Gandhinagar and Albert Einstein Institute, Golm, where parts of this work were done, for warm hospitality. SC and KP would like to thank T. Padmanabhan, K. Lochan, Dean Carmi and Pratik Rath for useful discussions. We would also like to thank the referee for his/her comments which have helped to improve the manuscript.

\section{Appendices}

\section{A Projections of the Riemann tensor near a null surface}

In this appendix, we shall derive the expressions of the projections of the Riemann tensor near a null surface, which will be necessary for our work. We shall start by introducing the relevant geometrical quantities near the null surface.

Let us consider a null surface in a $D$-dimensional spacetime represented by $\phi(x)=\phi_{0}$, for some scalar function $\phi(x)$ and some $\phi_{0}=$ constant. Other $\phi=$ constant surfaces need not be null, i.e., the null surface under consideration need not be the member of a null foliation. (We shall assume in the main text that all $\phi=$ constant surfaces are null. But the results in this appendix are derived without this assumption.) A null normal to the surface will have the form $\ell_{a}=A(x) \nabla_{a} \phi(x)$, for some other scalar function $A(x)$. Unlike the case for a non-null surface, the normal to a null surface cannot be normalized to unity. Hence, without a criterion for choosing the scaling, we consider all values of $A$ to be equally valid. This is the reason we talk about 'a' null normal rather than 'the' null normal. (Note that, while we may always choose the normal vector field as $\ell_{a}=\nabla_{a} \phi$, it is not possible, in general, to start with an $\ell_{a}=A \nabla_{a} \phi$ and choose a different scalar field $\phi^{\prime}$ to obtain $\ell_{a}=\nabla_{a} \phi^{\prime}$. This is proved in Appendix B. We want our results to be valid for any choice of the null normal and hence we will work with a general $A$.)

\section{A.1 Induced metric}

The induced metric of a hypersurface embedded in spacetime is derived from the metric of the spacetime by restricting its action to vectors tangent to the hypersurface. Although, strictly speaking, the induced metric acts on the lower-dimensional tangent space of the hypersurface, we can construct an 'induced metric' for the full manifold - a covariant 2-tensor acting on the tangent space of the whole manifold - by demanding that it annihilates the components of a vector normal to the hypersurface and coincides with the induced metric in its action on the components tangential to the hypersuface. We also take it to be a projector. This object is also called the orthogonal projector on the hypersurface. For a non-null hypersurface, the orthogonal projector is

$$
h_{a b}=g_{a b}-\epsilon n_{a} n_{b},
$$

where $n_{a}$ is the normalized normal and $\epsilon$ is -1 or +1 depending on whether the surface is timelike or spacelike. (The timelike case is more often found in textbooks, see $[5,72]$.) In this paper, we shall use the term 'induced metric' to mean the orthogonal projector. If we have occasion to use the lower-dimensional version, we shall make it amply clear.

Since one cannot construct a normalized normal for the null surface, it is not possible to define an induced metric in the above manner. The object $i_{a b}=g_{a b}+\lambda \ell_{a} \ell_{b}$ would not work for any finite $\lambda$ since this is not a projector:

$$
i_{b}^{a} i_{c}^{b}=\left(\delta_{b}^{a}+\lambda \ell^{a} \ell_{b}\right)\left(\delta_{c}^{b}+\lambda \ell^{b} \ell_{c}\right)=\delta_{c}^{a}+2 \lambda \ell^{a} \ell_{c} \neq i_{c}^{a} .
$$

The standard solution to the above problem is as follows: we choose an auxiliary null vector $k^{a}$ satisfying the relation $k^{a} \ell_{a}=-1$ ( [63,64], also see Appendix A.3 in arXiv version of [12]). Then, 
the induced metric on the null hypersurface is chosen as the object

$$
q_{a b} \equiv g_{a b}+\ell_{a} k_{b}+k_{a} \ell_{b} .
$$

Once can check that this is a projector. Note that it is orthogonal to both $\ell^{a}$ and $k^{a}$, and hence its restriction to the null surface is a codimension- 2 metric operating on the codimension- 2 vector space orthogonal to $k^{a}$ and tangent to the null surface. In the case where these codimension-2 vector spaces may be stitched together to form the tangent spaces of codimension-2 surfaces lying on the null surface, it is the metric on those codimension-2 surfaces. Following [73], a metric is a symmetric and non-degenerate tensor of type $(0,2)$. In the null case, we cannot define a nondegenerate metric on the null surface because of the presence of a null direction on the surface. But we can have a non-degenerate metric when we eliminate the null direction and focus on codimension-2 surfaces on the null surface. We will soon choose $k^{a}$ to be hypersurface-orthogonal in order to define connection and curvature on the null surface without introducing torsion.

\section{A.2 Induced connection and covariant derivative}

For an arbitrary tensor $T_{c d . . .}^{a b . .}$ that gives zero on contracting any index, up or down, with $\boldsymbol{\ell}$ or $\mathbf{k}$, the intrinsic covariant derivative $D$ on these codimension-2 surfaces may be defined as

$$
D_{a} T_{d e \ldots}^{b c \ldots} \equiv q_{a}^{i} q_{j}^{b} q_{k}^{c} q_{d}^{l} q_{e}^{m} \ldots \nabla_{i} T_{l m \ldots}^{j k \ldots},
$$

analogous to the non-null case [5]. Here $\nabla$ is the usual covariant derivative of the parent manifold. To see whether the covariant derivative operator $D$ defined in Eq. (84) is indeed a valid one, let us check each of the five criteria given in [73] when operating on tensors orthogonal to $\boldsymbol{\ell}$ or $\mathbf{k}$. These five conditions are

1. Linearity: This covariant derivative operator $D$ is certainly linear.

2. Leibnitz rule: $D$ satisfies the Leibnitz rule, as can be easily checked using its definition.

3. Commutativity with contraction: The operator $D$ also commutes with contraction, i.e., contraction with delta function of the space orthogonal to $k^{a}$ on the null surface. In working with indices of the whole manifold, this delta function is represented by $q_{b}^{a}=\delta_{b}^{a}+k^{a} \ell_{b}+\ell^{a} k_{b}$, where the extra terms are precisely to get rid of the components of the tensor not along the codimension-2 surfaces. [This can be easily seen using the canonical null basis and its dual basis (see Appendix A.3 in arXiv version of [12]), suitably extended to $D$ dimensions.] But note that we have already restricted ourselves to tensors orthogonal to $\boldsymbol{\ell}$ or $\mathbf{k}$. For such tensors, $\delta_{b}^{a}$ and $q_{b}^{a}$ are equivalent in considering commutativity with contraction.

4. Consistency with the notion of tangent vectors as directional derivatives on scalar fields: For a vector $t^{a}$ tangent to the codimension-2 surfaces on the null surface (i.e., such that $\ell_{a} t^{a}=0$ and $t_{a} k^{a}=0$ ), we have, for a scalar field $f$, the following relation:

$$
t^{a} D_{a} f=t^{a} q_{a}^{b} \nabla_{b} f=t^{a} \nabla_{a} f=t^{a} \partial_{a} f .
$$

Thus, $D_{a}$ indeed acts as a directional derivative when restricted to directions orthogonal to both $\ell^{a}$ and $k^{a}$.

5. Torsion free: For all scalars $f$, we should have $D_{a} D_{b} f=D_{b} D_{a} f$. In the present context, we have

$$
\begin{aligned}
D_{a} D_{b} f & =q_{a}^{c} q_{b}^{d} \nabla_{c}\left(D_{d} f\right)=q_{a}^{c} q_{b}^{d} \nabla_{c}\left(q_{d}^{e} \nabla_{e} f\right)=q_{a}^{c} q_{b}^{d} q_{d}^{e} \nabla_{c} \nabla_{e} f+q_{a}^{c} q_{b}^{d} \nabla_{e} f \nabla_{c} q_{d}^{e} \\
& =q_{a}^{c} q_{b}^{e} \nabla_{c} \nabla_{e} f+q_{a}^{c} q_{b}^{d} \nabla_{e} f \nabla_{c}\left(k^{e} \ell_{d}+\ell^{e} k_{d}\right) \\
& =q_{a}^{c} q_{b}^{e} \nabla_{c} \nabla_{e} f+q_{a}^{c} q_{b}^{d} \nabla_{e} f\left(k^{e} \nabla_{c} \ell_{d}+\ell^{e} \nabla_{c} k_{d}\right) .
\end{aligned}
$$

Thus,

$$
D_{a} D_{b} f-D_{b} D_{a} f=q_{a}^{c} q_{b}^{d}\left[k^{e} \nabla_{e} f\left(\nabla_{c} \ell_{d}-\nabla_{d} \ell_{c}\right)+\left(\ell^{e} \nabla_{e} f\right)\left(\nabla_{c} k_{d}-\nabla_{d} k_{c}\right)\right]
$$




$$
=k^{e} \nabla_{e} f\left(\Theta_{a b}-\Theta_{b a}\right)+\left(\ell^{e} \nabla_{e} f\right) q_{a}^{c} q_{b}^{d}\left(\nabla_{c} k_{d}-\nabla_{d} k_{c}\right),
$$

where we have used the expression for the second fundamental form on the null surface,

$$
\Theta_{c d} \equiv q_{c}^{m} q_{d}^{k} \nabla_{m} \ell_{k}
$$

In an identical manner, it is possible to define the corresponding quantity associated with the auxiliary null vector $k^{a}$ as

$$
\Psi_{c d} \equiv q_{c}^{m} q_{d}^{n} \nabla_{m} k_{n} .
$$

Using $\ell_{a}=A \nabla_{a} \phi$, one can prove that $\Theta_{a b}$ is symmetric (see Appendix A.3 in arXiv version of [12]). So we obtain

$$
D_{a} D_{b} f-D_{b} D_{a} f=\ell^{e} \nabla_{e} f\left(\Psi_{c d}-\Psi_{d c}\right) .
$$

This will be zero for arbitrary $f$ only if $\Psi_{a b}$ is also symmetric. This can be achieved if we take $k_{a}$ to be hypersurface-orthogonal, i.e.,

$$
k_{a}=B \nabla_{a} \psi,
$$

with $B$ and $\psi$ being two scalar functions. (The proof that $\Theta_{c d}$ is symmetric automatically tells us that $\Psi_{c d}$ would also be symmetric, since $q_{a b}$ is symmetric with respect to an interchange of $\ell$ and $k$.) Since we do not intend to deal with torsion in this paper, we shall make the assumption that $k_{a}$ is also hypersurface-orthogonal. In this case, there are two foliations, represented by the scalars $\phi$ and $\psi$ with normals $\ell_{a}$ and $k_{a}$, respectively. A particular member of the $\phi$-foliation, say $\phi=\phi_{0}$, is null while the auxiliary foliation due to the scalar $\psi$ is taken to be composed of all null surfaces. In fact, the auxiliary foliation can be constructed by sending out null rays along $k^{a}$ from our fiducial null surface (see the description of Gaussian null coordinates [74-77] in Appendix C of [12]). The object $q_{a b}$ then represents the induced metric on the codimension- 2 surfaces formed by the intersection of the members of the $\psi$-foliation with the $\phi=\phi_{0}$ null surface.

\section{A.3 Intrinsic (Gaussian) curvature}

Using the above definition of the induced covariant derivative and any vector $X^{a}$ satisfying $X^{a} \ell_{a}=$ 0 and $X^{a} k_{a}=0$, we can define the intrinsic Riemann tensor on the codimension- 2 surfaces orthogonal to $k^{a}$ on the null surface, following [73] and in analogy with the non-null case discussed in $[5,72]$, as

$$
{ }^{\left({ }^{D-2)}\right.} R_{a b c d} X^{b}=D_{c} D_{d} X_{a}-D_{d} D_{c} X_{a} .
$$

Note that what is defined above is the $D$-dimensional object. The components corresponding to the directions on the codimension-2 surfaces give the intrinsic curvature of those surfaces. See [64] for a formulation where these components are separated out and written using an appropriate set of basis vectors.

We refer the reader to Appendix A.3 of the arxiv version of [12] for more on the geometrical quantities and identities related to a null surface. Let us now carry out the derivation of the various identities relating the curvature tensor in $D$ spacetime dimensions to the curvature tensor on the codimension-2 surfaces that lie on the null surface and are orthogonal to $k^{a}$.

Before starting on the derivations of the projections necessary for our calculation, let us first compare the null case with the case of projections near a non-null hypersurface. For a non-null hypersuface, there are three relations - the Gauss, the Codazzi-Mainardi and the Ricci relationscorresponding to none, one and two indices being projected on the normal, respectively, and the rest being projected on the hypersurface $[5,72,78]$. In the null case, we are dealing with the projector $q_{b}^{a}$, which is, in actuality, a projector on codimension- 2 surfaces, the ones orthogonal to $k^{a}$ on the null surface, masquerading as a projector on the codimension-1 null surface. What 
this entails is the involvement of two normals, $\ell^{a}$ and $k^{a}$, rather than one. Let us enumerate the possibilities of projections with $\ell^{a}, k^{a}$ and $q_{b}^{a}$. The analogue of the Gauss relation would be the contraction of all indices of the Riemann tensor with $q_{b}^{a}$ 's, just having to remember that we are projecting on the codimension- 2 surfaces. (If we were just dealing with the codimension-1 null surface, even a projection on $\ell^{a}$ would be tangential to the null surface.) For the rest of the relations to follow, we shall assume that the remaining indices are contracted with $q_{b}^{a}$ 's. The Codazzi-Mainardi relation is now two in number, one for contracting a single index with $\ell^{a}$ and another for contracting it with $k^{a}$. Moving on to the Ricci relation, the options of contracting two indices with the normals are four. One could contract two of the indices that are not antisymmetric among themselves with two $\ell^{a}$ 's or two $k^{a}$ 's, or one could take one $\ell^{a}$ and one $k^{a}$ and contract it either with two indices that are either antisymmetric among themselves or are not. In this case, we have the options of choosing three or four indices to be contracted with the normals. For the case of three, choose the case of two $\ell^{a}$ 's or two $k^{a}$ 's from the Ricci case and contract one of the two remaining indices (doesn't matter which one) with the other normal. Thus, there are two cases for three contractions with the normal. Finally, the completely normal case of contraction with four normals would require two $\ell^{a}$ 's and two $k^{a}$ 's, with the two of a pair occupying indices that are not antisymmetric. All such cases are the same, giving just one such completely normal case of contraction with four normals.

Thus, there are ten contractions of the Riemann tensor possible with the objects $\ell^{a}, k^{a}$ and $q_{b}^{a}$. Out of these ten, we are only going to consider the three obtained without using $k^{a}$, since these are the ones we will require for our calculations of the boundary term.

\section{A.4 Various projections of the Riemann tensor}

\section{A.4.1 Identity involving $q_{a}^{p} q_{b}^{q} q_{c}^{r} q_{d}^{s} R_{p q r s}$ : the null Gauss relation}

The first identity corresponds to the complete projection of the curvature tensor on the null hypersurface using the induced metric associated with the co-dimension two surfaces. For this purpose, we start with Eq. (92) and expand according to Eq. (84) to obtain

$$
\begin{aligned}
{ }^{(D-2)} R_{a b c d} X^{b} & =\left\{q_{c}^{m} q_{d}^{k} q_{a}^{l} \nabla_{m}\left(q_{k}^{i} q_{l}^{j} \nabla_{i} X_{j}\right)\right\}-\{c \leftrightarrow d\} \\
& =\left\{q_{c}^{m} q_{d}^{i} q_{a}^{j} \nabla_{m} \nabla_{i} X_{j}+q_{c}^{m} q_{d}^{k} q_{a}^{j} \nabla_{i} X_{j} \nabla_{m} q_{k}^{i}+q_{c}^{m} q_{d}^{i} q_{a}^{l} \nabla_{i} X_{j} \nabla_{m} q_{l}^{j}\right\}-\{c \leftrightarrow d\},
\end{aligned}
$$

where $\{c \leftrightarrow d\}$ represents the expression obtained by interchanging $c$ and $d$ in the expression inside the first set of curly brackets. The second term in the above expression may be rewritten as follows:

$$
\begin{aligned}
q_{c}^{m} q_{d}^{k} q_{a}^{j} \nabla_{i} X_{j} \nabla_{m} q_{k}^{i} & =q_{c}^{m} q_{d}^{k} q_{a}^{j} \nabla_{i} X_{j} \nabla_{m}\left(\ell^{i} k_{k}+k^{i} \ell_{k}\right) \\
& =q_{c}^{m} q_{d}^{k} q_{a}^{j} \nabla_{i} X_{j}\left(\ell^{i} \nabla_{m} k_{k}+k^{i} \nabla_{m} \ell_{k}\right) \\
& =q_{a}^{j}\left[\Psi_{c d}(\ell . \nabla) X_{j}+\Theta_{c d}(\mathbf{k} . \nabla) X_{j}\right],
\end{aligned}
$$

where we have used Eq. (88) and Eq. (89). Proceeding in an identical manner, the third term in Eq. (93) may be rewritten as

$$
\begin{aligned}
q_{c}^{m} q_{d}^{i} q_{a}^{l} \nabla_{i} X_{j} \nabla_{m} q_{l}^{j} & =q_{c}^{m} q_{d}^{i} q_{a}^{l} \nabla_{i} X_{j}\left(\ell^{j} \nabla_{m} k_{l}+k^{j} \nabla_{m} \ell_{l}\right) \\
& =q_{d}^{i}\left[\Psi_{c a} \ell^{j} \nabla_{i} X_{j}+\Theta_{c a} k^{j} \nabla_{i} X_{j}\right] .
\end{aligned}
$$

Substituting Eq. (94) and Eq. (95) in Eq. (93), we obtain

$$
\begin{aligned}
{ }^{(D-2)} R_{a b c d} X^{b}= & \left\{q_{c}^{m} q_{d}^{i} q_{a}^{j} \nabla_{m} \nabla_{i} X_{j}+q_{a}^{j}\left[\Psi_{c d}(\ell \cdot \nabla) X_{j}+\Theta_{c d}(\mathbf{k} . \nabla) X_{j}\right]+q_{d}^{i}\left[\Psi_{c a} \ell^{j} \nabla_{i} X_{j}+\Theta_{c a} k^{j} \nabla_{i} X_{j}\right]\right\} \\
& -\{c \leftrightarrow d\} \\
= & q_{c}^{m} q_{d}^{i} q_{a}^{j}\left(\nabla_{m} \nabla_{i}-\nabla_{i} \nabla_{m}\right) X_{j}
\end{aligned}
$$




$$
\begin{aligned}
& \quad+q_{a}^{j}\left[\left(\Psi_{c d}-\Psi_{d c}\right)(\ell . \nabla) X_{j}+\left(\Theta_{c d}-\Theta_{d c}\right)(\mathbf{k} . \nabla) X_{j}\right] \\
& \quad+\left[q_{d}^{i}\left(\Psi_{c a} \ell^{j} \nabla_{i} X_{j}+\Theta_{c a} k^{j} \nabla_{i} X_{j}\right)\right]-[c \leftrightarrow d] \\
& =q_{c}^{m} q_{d}^{i} q_{a}^{j} R_{j n m i} X^{n} \\
& \quad+\left[q_{d}^{i}\left(-\Psi_{c a} X_{j} \nabla_{i} \ell^{j}-\Theta_{c a} X_{j} \nabla_{i} k^{j}\right)\right]-[c \leftrightarrow d],
\end{aligned}
$$

where, as in Appendix A.2, we have used the symmetry of $\Theta_{a b}$ and $\Psi_{a b}$. Next we would like to remove the arbitrary vector $X^{a}$ from both sides. Remember that $X^{a}$ here is a vector that is orthogonal to $\ell^{a}$ and $k^{a}$, i.e., a vector tangential to the codimension- 2 surfaces on the null surface orthogonal to $k^{a}$. The above identity is not valid if $X^{a}$ is replaced by a general vector in the $D$-dimensional spacetime. Thus, we are not allowed to just cancel $X^{a}$ from both sides. But, given any vector $Y^{a}$ in the $D$-dimensional spacetime, we can construct a vector $X^{a}$ orthogonal to $\ell^{a}$ and $k^{a}$ as $X^{a}=q_{b}^{a} Y^{b}$. Thus, the above identity can be written in terms of an arbitrary $D$-dimensional vector $Y^{a}$ as

$$
\begin{aligned}
{ }^{(D-2)} R_{a b c d} q_{e}^{b} Y^{e} & =q_{c}^{m} q_{d}^{i} q_{a}^{j} q_{e}^{n} R_{j n m i} Y^{e}+\left[q_{d}^{i}\left(-\Psi_{c a} \nabla_{i} \ell^{j}-\Theta_{c a} \nabla_{i} k^{j}\right) q_{j e} Y^{e}\right]-[c \leftrightarrow d] \\
& =q_{c}^{m} q_{d}^{i} q_{a}^{j} q_{e}^{n} R_{j n m i} Y^{e}+\left[\left(-\Psi_{c a} \Theta_{d e}-\Theta_{c a} \Psi_{d e}\right) Y^{e}\right]-[c \leftrightarrow d] .
\end{aligned}
$$

Dropping the arbitrary vector $Y^{e}$, we obtain

$$
{ }^{\left({ }^{D-2)}\right.} R_{a b c d} q_{e}^{b}=q_{c}^{m} q_{d}^{i} q_{a}^{j} q_{e}^{n} R_{j n m i}-\left[\left(\Psi_{c a} \Theta_{d e}+\Theta_{c a} \Psi_{d e}\right)-(c \leftrightarrow d)\right] .
$$

Now, we make the assumption that ${ }^{(D-2)} R_{a b c d}$ is orthogonal to $\ell^{a}$ and $k^{a}$ on the index $b$. (It is already orthogonal to these vectors on the other indices, as is obvious from the right-hand side of the above equation.) Looking back at Eq. (92), we see that this equation only tells us the behaviour of ${ }^{(D-2)} R_{a b c d}$ when the index $b$ is contracted with vectors orthogonal to $\ell^{a}$ and $k^{a}$. It does not tell us anything about the contraction of this index with $\ell^{a}$ or $k^{a}$. So we make the simplest assumption, not distinguishing $b$ in this respect from the other indices. With this assumption, only the $\delta_{e}^{b}$ part of $q_{e}^{b}$ in Eq. (98) survives and we obtain the null version of the Gauss relation [72]:

$$
{ }^{(D-2)} R_{a b c d}=q_{a}^{j} q_{b}^{n} q_{c}^{m} q_{d}^{i} R_{j n m i}-\left[\left(\Psi_{c a} \Theta_{d b}+\Theta_{c a} \Psi_{d b}\right)-(c \leftrightarrow d)\right]
$$

or

$$
q_{a}^{j} q_{b}^{n} q_{c}^{m} q_{d}^{i} R_{j n m i}={ }^{(D-2)} R_{a b c d}+\left[\left(\Psi_{c a} \Theta_{d b}+\Theta_{c a} \Psi_{d b}\right)-(c \leftrightarrow d)\right] .
$$

Using Eq. (99), it can be checked that ${ }^{(D-2)} R_{a b c d}$ satisfies all the algebraic identities (the identities without any covariant derivatives) of the Riemann tensor.

\section{A.4.2 Identity involving $\ell^{p} q_{b}^{q} q_{c}^{r} q_{d}^{s} R_{p q r s}$ : the null Codazzi-Mainardi relation}

The next identity we derive will have one of the indices of the Riemann tensor contracted with the null normal $\ell^{a}$ and the other three projected on the null surface using $q_{b}^{a}$. Note that we need to derive only the case of the first index contracted with the null normal, since an instance of the null normal contracted with another index can be converted to this case using the symmetries of the Riemann tensor. We start with

$$
\begin{aligned}
\ell^{a} q_{p}^{b} q_{q}^{c} q_{r}^{d} R_{a b c d}= & q_{p}^{b} q_{q}^{c} q_{r}^{d}\left(\nabla_{d} \nabla_{c} \ell_{b}-\nabla_{c} \nabla_{d} \ell_{b}\right)=\left(q_{p}^{b} q_{q}^{c} q_{r}^{d} \nabla_{d} \nabla_{c} \ell_{b}\right)-(q \leftrightarrow r) \\
= & \left(q_{r}^{d} q_{m}^{b} q_{p}^{m} q_{n}^{c} q_{q}^{n} \nabla_{d} \nabla_{c} \ell_{b}\right)-(q \leftrightarrow r) \\
= & {\left[q_{r}^{d} q_{p}^{m} q_{q}^{n} \nabla_{d}\left(q_{m}^{b} q_{n}^{c} \nabla_{c} \ell_{b}\right)-q_{r}^{d} q_{p}^{m} q_{q}^{n} \nabla_{d}\left(q_{m}^{b} q_{n}^{c}\right) \nabla_{c} \ell_{b}\right]-[q \leftrightarrow r] } \\
= & {\left[D_{r} \Theta_{p q}-q_{r}^{d} q_{p}^{b} q_{q}^{n} \nabla_{d}\left(q_{n}^{c}\right) \nabla_{c} \ell_{b}-q_{r}^{d} q_{p}^{m} q_{q}^{c} \nabla_{d}\left(q_{m}^{b}\right) \nabla_{c} \ell_{b}\right]-[q \leftrightarrow r] } \\
= & {\left[D_{r} \Theta_{p q}-q_{r}^{d} q_{p}^{b} q_{q}^{n}\left(k^{c} \nabla_{d} \ell_{n}+\ell^{c} \nabla_{d} k_{n}\right) \nabla_{c} \ell_{b}-q_{r}^{d} q_{p}^{m} q_{q}^{c}\left(k^{b} \nabla_{d} \ell_{m}+\ell^{b} \nabla_{d} k_{m}\right) \nabla_{c} \ell_{b}\right] } \\
& -[q \leftrightarrow r] \\
= & {\left[D_{r} \Theta_{p q}-q_{p}^{b}\left(k^{c} \Theta_{r q}+\ell^{c} \Psi_{r q}\right) \nabla_{c} \ell_{b}-q_{q}^{c}\left(k^{b} \Theta_{r p}+\ell^{b} \Psi_{r p}\right) \nabla_{c} \ell_{b}\right]-[q \leftrightarrow r] }
\end{aligned}
$$




$$
=\left[D_{r} \Theta_{p q}-q_{q}^{c}\left(k^{b} \Theta_{r p}+\ell^{b} \Psi_{r p}\right) \nabla_{c} \ell_{b}\right]-[q \leftrightarrow r],
$$

where, in the second-last step, we have used the fact that $\Theta_{r q}$ and $\Psi_{r q}$ are symmetric. The expression $q_{q}^{c} \ell^{b} \nabla_{c} \ell_{b}=q_{q}^{c}\left(\nabla_{c} \ell^{2}\right) / 2=\left(D_{q} \ell^{2}\right) / 2$ is zero at the null surface, since this is a derivative along the codimension-2 surfaces orthogonal to $k^{a}$ on the null surface and $\ell^{2}$ is zero all over the null surface. Another way to see this is by substituting $\ell_{b}=A \nabla_{b} \phi$ and simplifying:

$$
\begin{aligned}
q_{q}^{c} \ell^{b} \nabla_{c} \ell_{b} & =q_{q}^{c} \ell^{b} \nabla_{c}\left(A \nabla_{b} \phi\right)=q_{q}^{c} \ell^{b} \ell_{b} \partial_{c} \ln A+q_{q}^{c} \ell^{b} A \nabla_{c} \nabla_{b} \phi=q_{q}^{c} \ell^{b} A \nabla_{b} \nabla_{c} \phi=q_{q}^{c} \ell^{b} A \nabla_{b}\left(\frac{\ell_{c}}{A}\right) \\
& =q_{q}^{c} \ell^{b} \nabla_{b} \ell_{c}=q_{q}^{c} \kappa \ell_{c}=0,
\end{aligned}
$$

where we have used $\ell^{b} \ell_{b}=0$ and the formula

$$
\ell^{a} \nabla_{a} \ell_{b}=\kappa \ell_{b},
$$

with $\kappa$ called the non-affinity coefficient (see Appendix A.3 in arXiv version of [12]). Thus, we obtain

$$
\begin{aligned}
\ell^{a} q_{p}^{b} q_{q}^{c} q_{r}^{d} R_{a b c d} & =\left(D_{r} \Theta_{p q}-q_{q}^{c} k^{b} \Theta_{r p} \nabla_{c} \ell_{b}\right)-(q \leftrightarrow r) \\
\Rightarrow \ell^{a} q_{p}^{b} q_{q}^{c} q_{r}^{d} R_{a b c d} & =D_{r} \Theta_{p q}-D_{q} \Theta_{p r}+\left(q_{r}^{c} \Theta_{q p}-q_{q}^{c} \Theta_{r p}\right) k^{b} \nabla_{c} \ell_{b},
\end{aligned}
$$

which is the null version of the Codazzi-Mainardi relation [72]. (The name of Mainardi is often absent in physics literature.)

\section{A.4.3 Identities involving $q_{a}^{p} \ell^{q} q_{b}^{r} \ell^{s} R_{p q r s}$ : the null Ricci relation}

The last expression we shall derive is of the decomposition $q_{p}^{a} \ell^{b} q_{q}^{c} \ell^{d} R_{a b c d}$. This is the remaining projection of the Riemann tensor using only $q_{b}^{a}$ and $\ell^{a}$, since the symmetries of the Riemann tensor ensure that contracting more than two indices with the null normal will give zero. In our case, though, we also have the vector $k^{a}$ and other projections can be carried out with $k^{a}$ in the mix, as we have indicated before. But these are not required for our purpose and so we shall not be deriving them here.

We start by decomposing $\nabla_{a} \ell_{b}$ :

$$
\begin{aligned}
\nabla_{a} \ell_{b}= & \left(q_{a}^{c}-k^{c} \ell_{a}-\ell^{c} k_{a}\right)\left(q_{b}^{d}-k^{d} \ell_{b}-\ell^{d} k_{b}\right) \nabla_{c} \ell_{d} \\
= & q_{a}^{c} q_{b}^{d} \nabla_{c} \ell_{d}-q_{a}^{c} k^{d} \ell_{b} \nabla_{c} \ell_{d}-q_{a}^{c} \ell^{d} k_{b} \nabla_{c} \ell_{d}-k^{c} \ell_{a} q_{b}^{d} \nabla_{c} \ell_{d}+k^{c} \ell_{a} k^{d} \ell_{b} \nabla_{c} \ell_{d} \\
& +k^{c} \ell_{a} \ell^{d} k_{b} \nabla_{c} \ell_{d}-\ell^{c} k_{a} q_{b}^{d} \nabla_{c} \ell_{d}+\ell^{c} k_{a} k^{d} \ell_{b} \nabla_{c} \ell_{d}+\ell^{c} k_{a} \ell^{d} k_{b} \nabla_{c} \ell_{d} \\
= & \Theta_{a b}-q_{a}^{c} k^{d} \ell_{b} \nabla_{c} \ell_{d}-k^{c} \ell_{a} q_{b}^{d} \nabla_{c} \ell_{d}+k^{c} \ell_{a} k^{d} \ell_{b} \nabla_{c} \ell_{d} \\
& +k^{c} \ell_{a} \ell^{d} k_{b} \nabla_{c} \ell_{d}-\kappa k_{a} \ell_{b} \\
= & \Theta_{a b}-q_{a}^{c} k^{d} \ell_{b} \nabla_{c} \ell_{d}-k^{c} \ell_{a} q_{b}^{d} \nabla_{c} \ell_{d}+k^{c} \ell_{a} k^{d} \ell_{b} \nabla_{c} \ell_{d}-\tilde{\kappa} \ell_{a} k_{b}-\kappa k_{a} \ell_{b},
\end{aligned}
$$

where we have used Eq. (102), Eq. (103) and the fact that $\ell^{c} \ell^{d} \nabla_{c} \ell_{d}=\ell^{c} \partial_{c}\left(\ell_{d} \ell^{d} / 2\right)=0$ since the derivative is along the null surface where $\ell_{d} \ell^{d}=0$ throughout. We have also used the definition (see Appendix A.3 in arXiv version of [12])

$$
\tilde{\kappa} \equiv-\frac{k^{a}}{2} \partial_{a}\left(\ell^{b} \ell_{b}\right)=-k^{a} \ell^{b} \nabla_{a} \ell_{b} .
$$

The object $\tilde{\kappa}$ may be called the "nullness gradient" since it measures how $\ell^{a} \ell_{a}$ varies as one moves away from the null surface. This is related to $\kappa$ by the relation

$$
\kappa=\ell^{a} \partial_{a} \ln A+\tilde{\kappa} .
$$

Note that when $A$ does not vary in the null direction $\ell^{a}$, we shall have

$$
\kappa=\tilde{\kappa} .
$$


Regrouping the terms, it is possible to write down Eq. (105) in a nice form, resulting in

$$
\nabla_{a} \ell_{b}=\Theta_{a b}-\left(k^{d} \nabla_{c} \ell_{d}\right) q_{a}^{c} \ell_{b}-\left(k^{c} \nabla_{c} \ell_{d}\right) \ell_{a} q_{b}^{d}+\left(k^{c} k^{d} \nabla_{c} \ell_{d}\right) \ell_{a} \ell_{b}-\tilde{\kappa} \ell_{a} k_{b}-\kappa k_{a} \ell_{b} .
$$

This equation has $\nabla_{a} \ell_{b}$ decomposed along $q_{b}^{a}, \ell_{a}$ and $k_{a}$. Using Eq. (109), the projection $q_{p}^{a} \ell^{b} q_{q}^{c} \ell^{d} R_{a b c d}$ can be decomposed as follows:

$$
\begin{aligned}
& R_{a b c d} q_{p}^{a} \ell^{b} q_{q}^{c} \ell^{d}=q_{p}^{a} q_{q}^{c} \ell^{d}\left(\nabla_{c} \nabla_{d}-\nabla_{d} \nabla_{c}\right) \ell_{a} \\
& =q_{p}^{a} q_{q}^{c} \ell^{d} \nabla_{c}\left[\Theta_{d a}-\left(k^{i} \nabla_{j} \ell_{i}\right) q_{d}^{j} \ell_{a}-\left(k^{i} \nabla_{i} \ell_{j}\right) \ell_{d} q_{a}^{j}+\left(k^{i} k^{j} \nabla_{i} \ell_{j}\right) \ell_{d} \ell_{a}-\tilde{\kappa} \ell_{d} k_{a}-\kappa k_{d} \ell_{a}\right] \\
& \quad-q_{p}^{a} q_{q}^{c} \ell^{d} \nabla_{d}\left[\Theta_{c a}-\left(k^{i} \nabla_{j} \ell_{i}\right) q_{c}^{j} \ell_{a}-\left(k^{i} \nabla_{i} \ell_{j}\right) \ell_{c} q_{a}^{j}+\left(k^{i} k^{j} \nabla_{i} \ell_{j}\right) \ell_{c} \ell_{a}-\tilde{\kappa} \ell_{c} k_{a}-\kappa k_{c} \ell_{a}\right] \\
& =q_{p}^{a} q_{q}^{c} \ell^{d} \nabla_{c}\left[\Theta_{d a}-\left(k^{i} \nabla_{i} \ell_{j}\right) \ell_{d} q_{a}^{j}-\kappa k_{d} \ell_{a}\right] \\
& \quad-q_{p}^{a} q_{q}^{c} \ell^{d} \nabla_{d}\left[\Theta_{c a}-\left(k^{i} \nabla_{j} \ell_{i}\right) q_{c}^{j} \ell_{a}-\left(k^{i} \nabla_{i} \ell_{j}\right) \ell_{c} q_{a}^{j}\right] \\
& =q_{p}^{a} q_{q}^{c} \ell^{d}\left(\nabla_{c} \Theta_{d a}-\nabla_{d} \Theta_{c a}\right)-\left(k^{i} \nabla_{i} \ell_{j}\right) q_{p}^{a} q_{q}^{c} q_{a}^{j} \ell^{d} \nabla_{c} \ell_{d}+\kappa q_{p}^{a} q_{q}^{c} \nabla_{c} \ell_{a} \\
& \quad+\left(k^{i} \nabla_{j} \ell_{i}\right) q_{c}^{j} q_{p}^{a} q_{q}^{c} \ell^{d} \nabla_{d} \ell_{a}+q_{p}^{a} q_{q}^{c}\left(k^{i} \nabla_{i} \ell_{j}\right) q_{a}^{j} \ell^{d} \nabla_{d} \ell_{c} \\
& =q_{p}^{a} q_{q}^{c} \ell^{d}\left(\nabla_{c} \Theta_{d a}-\nabla_{d} \Theta_{c a}\right)+\kappa q_{p}^{a} q_{q}^{c} \nabla_{c} \ell_{a}=q_{p}^{a} q_{q}^{c} \ell^{d}\left(\nabla_{c} \Theta_{d a}-\nabla_{d} \Theta_{c a}\right)+\kappa \Theta_{p q},
\end{aligned}
$$

where, in the third step, some terms have dropped out because when we act the covariant derivative on them and use the Leibniz's rule for the action of a derivative on a product, we see that the resulting terms are zero when contracted with the factors outside. We have also used Eq. (102) and Eq. (103). We have thus obtained the following simple relation:

$$
R_{a b c d} q_{p}^{a} \ell^{b} q_{q}^{c} \ell^{d}=q_{p}^{a} q_{q}^{c} \ell^{d}\left(\nabla_{c} \Theta_{d a}-\nabla_{d} \Theta_{c a}\right)+\kappa \Theta_{p q} .
$$

A little more manipulation leads to another nice form:

$$
\begin{aligned}
R_{a b c d} q_{p}^{a} \ell^{b} q_{q}^{c} \ell^{d} & =q_{p}^{a} q_{q}^{c} \ell^{d}\left(\nabla_{c} \Theta_{d a}-\nabla_{d} \Theta_{c a}\right)+\kappa \Theta_{p q} \\
& =q_{p}^{a} q_{q}^{c} \nabla_{c}\left(\ell^{d} \Theta_{d a}\right)-q_{q}^{c} \Theta_{d p} \nabla_{c} \ell^{d}-q_{p}^{a} q_{q}^{c} \ell^{d} \nabla_{d} \Theta_{c a}+\kappa \Theta_{p q} \\
\Rightarrow R_{a b c d} q_{p}^{a} \ell^{b} q_{q}^{c} \ell^{d} & =-\Theta_{p d} \Theta_{q}^{d}-q_{p}^{a} q_{q}^{c} \ell^{d} \nabla_{d} \Theta_{c a}+\kappa \Theta_{p q} .
\end{aligned}
$$

It turns out that one can write the above expression in terms of the Lie derivative of $\Theta_{c a}$ along $\ell^{a}$, given in terms of covariant derivatives by the following formula:

$$
£_{\ell} \Theta_{c a}=\ell^{b} \nabla_{b} \Theta_{c a}+\Theta_{b a} \nabla_{c} \ell^{b}+\Theta_{c b} \nabla_{a} \ell^{b} .
$$

Substituting for the covariant derivative of $\Theta_{c a}$ along $\ell^{a}$ by the corresponding Lie derivative, Eq. (112) becomes

$$
\begin{aligned}
R_{a b c d} q_{p}^{a} \ell^{b} q_{q}^{c} \ell^{d} & =-\Theta_{p d} \Theta_{q}^{d}-q_{p}^{a} q_{q}^{c}\left(£_{\ell} \Theta_{c a}-\Theta_{b a} \nabla_{c} \ell^{b}-\Theta_{c b} \nabla_{a} \ell^{b}\right)+\kappa \Theta_{p q} \\
& =-\Theta_{p d} \Theta_{q}^{d}-q_{p}^{a} q_{q}^{c} £_{\ell} \Theta_{c a}+\Theta_{b p} \Theta_{q}^{b}+\Theta_{q b} \Theta_{p}^{b}+\kappa \Theta_{p q} \\
\Rightarrow R_{a b c d} q_{p}^{a} \ell^{b} q_{q}^{c} \ell^{d} & =-q_{p}^{a} q_{q}^{c} £_{\ell} \Theta_{c a}+\Theta_{p d} \Theta_{q}^{d}+\kappa \Theta_{p q} .
\end{aligned}
$$

We would like to use a particular form of the above equation where one index on $\Theta$ is up and the other is down. So we take Eq. (114) and manipulate it as follows:

$$
\begin{aligned}
R_{a b c d} q_{p}^{a} \ell^{b} q_{q}^{c} \ell^{d} & =-q_{p}^{a} q_{q}^{c} £_{\ell} \Theta_{c a}+\Theta_{p d} \Theta_{q}^{d}+\kappa \Theta_{p q} \\
& =-q_{p}^{a} q_{q}^{c} £_{\ell}\left(g_{c e} \Theta_{a}^{e}\right)+\Theta_{p d} \Theta_{q}^{d}+\kappa \Theta_{p q} \\
& =-q_{p}^{a} q_{q}^{c}\left(\Theta_{a}^{e} £_{\ell} g_{c e}+g_{c e} £_{\ell} \Theta_{a}^{e}\right)+\Theta_{p d} \Theta_{q}^{d}+\kappa \Theta_{p q} \\
& =-q_{p}^{a} q_{q}^{c}\left[\Theta_{a}^{e}\left(\nabla_{c} \ell_{e}+\nabla_{e} \ell_{c}\right)+g_{c e} £_{\ell}^{e} \Theta_{a}^{e}+\Theta_{p d} \Theta_{q}^{d}+\kappa \Theta_{p q}\right. \\
& =-q_{p}^{a} q_{q}^{c}\left[2 \Theta_{a}^{e} \Theta_{c e}+g_{c e} £_{\ell} \Theta_{a}^{e}\right]+\Theta_{p d} \Theta_{q}^{d}+\kappa \Theta_{p q} \\
& =-2 \Theta_{p}^{e} \Theta_{q e}-q_{p}^{a} q_{q e} £_{\ell} \Theta_{a}^{e}+\Theta_{p d} \Theta_{q}^{d}+\kappa \Theta_{p q} \\
\Rightarrow R_{a b c d} q_{p}^{a} \ell^{b} q_{q}^{c} \ell^{d} & =-q_{p}^{a} q_{q e} £_{\ell} \Theta_{a}^{e}-\Theta_{p}^{e} \Theta_{q e}+\kappa \Theta_{p q},
\end{aligned}
$$


where we have used the symmetry of $\Theta_{a b}$. This is the formula we will be using in our derivation of the boundary term. But for getting this into a form similar to the Ricci relation $[5,78]$, we have to take the $q$ 's inside the Lie derivative. We start with the formula for the action of the Lie derivative on $q_{b}^{a}$ 's:

$$
\begin{aligned}
£_{\ell} q_{b}^{a} & =\ell^{c} \nabla_{c} q_{b}^{a}-q_{b}^{c} \nabla_{c} \ell^{a}+q_{c}^{a} \nabla_{b} \ell^{c}=\ell^{c} \nabla_{c}\left(\delta_{b}^{a}+\ell^{a} k_{b}+k^{a} \ell_{b}\right)-q_{b}^{c} \nabla_{c} \ell^{a}+q_{c}^{a} \nabla_{b} \ell^{c} \\
& =\ell^{c} \nabla_{c}\left(\ell^{a} k_{b}+k^{a} \ell_{b}\right)-q_{b}^{c} \nabla_{c} \ell^{a}+q_{c}^{a} \nabla_{b} \ell^{c} .
\end{aligned}
$$

Instead of the previous result, Eq. (115), we start with Eq. (114). Taking the $q$ 's inside the Lie derivative in Eq. (114), we obtain

$$
\begin{aligned}
R_{a b c d} q_{p}^{a} \ell^{b} q_{q}^{c} \ell^{d}= & -£_{\ell} \Theta_{p q}+\left[\left(\Theta_{c a} q_{q}^{c} £_{\ell} q_{p}^{a}\right)+(p \leftrightarrow q)\right]+\Theta_{p d} \Theta_{q}^{d}+\kappa \Theta_{p q} \\
= & -£_{\ell} \Theta_{p q}+\Theta_{p d} \Theta_{q}^{d}+\kappa \Theta_{p q}+\left\{\Theta_{q a}\left[\ell^{c} \nabla_{c}\left(\ell^{a} k_{p}+k^{a} \ell_{p}\right)-q_{p}^{c} \nabla_{c} \ell^{a}+q_{c}^{a} \nabla_{p} \ell^{c}\right]\right\}+\{p \leftrightarrow q\} \\
= & -£_{\ell} \Theta_{p q}+\Theta_{p d} \Theta_{q}^{d}+\kappa \Theta_{p q}+\left\{\left[\Theta_{q a}\left(k_{p} \ell^{c} \nabla_{c} \ell^{a}+\ell_{p} \ell^{c} \nabla_{c} k^{a}\right)-\Theta_{q a} \Theta_{p}^{a}+\Theta_{q c} \nabla_{p} \ell^{c}\right]\right\} \\
& +\{p \leftrightarrow q\} \\
= & -£_{\ell} \Theta_{p q}+\kappa \Theta_{p q}-\Theta_{q a} \Theta_{p}^{a}+\left\{\left[\Theta_{q a}\left(k_{p} \kappa \ell^{a}+\ell_{p} \ell^{c} \nabla_{c} k^{a}\right)+\Theta_{q c}\left(q_{p}^{a}-\ell^{a} k_{p}-k^{a} \ell_{p}\right) \nabla_{a} \ell^{c}\right]\right\} \\
& +\{p \leftrightarrow q\} \\
= & -£_{\ell} \Theta_{p q}+\kappa \Theta_{p q}-\Theta_{q a} \Theta_{p}^{a}+\left\{\Theta_{q a} \ell_{p} \ell^{c} \nabla_{c} k^{a}+\Theta_{q c} \Theta_{p}^{c}-\Theta_{q c} \ell_{p} k^{a} \nabla_{a} \ell^{c}\right\}+\{p \leftrightarrow q\} \\
\Rightarrow R_{a b c d} q_{p}^{a} \ell^{b} q_{q}^{c} \ell^{d}= & -£_{\ell} \Theta_{p q}+\kappa \Theta_{p q}+\Theta_{q c} \Theta_{p}^{c}+\left(\Theta_{p a} \ell_{q}+\Theta_{q a} \ell_{p}\right)\left(\ell^{c} \nabla_{c} k^{a}-k^{c} \nabla_{c} \ell^{a}\right),
\end{aligned}
$$

where we have used the symmetry of $\Theta_{a b}$ and Eq. (103). Recognizing that $\ell^{b} \nabla_{b} k^{a}-k^{b} \nabla_{b} \ell^{a}=£_{\ell} k^{a}$ (the Lie bracket $[\boldsymbol{\ell}, \boldsymbol{k}]$ ), we can write the above equation as

$$
R_{a b c d} q_{p}^{a} \ell^{b} q_{q}^{c} \ell^{d}=-£_{\ell} \Theta_{p q}+\left(\Theta_{p a} \ell_{q}+\Theta_{q a} \ell_{p}\right) £_{\ell} k^{a}+\kappa \Theta_{p q}+\Theta_{q c} \Theta_{p}^{c} .
$$

\section{B Why $\ell_{a}=\nabla_{a} \phi^{\prime}$ is not the general case}

If we have $\ell_{a}=A \nabla_{a} \phi$, is it possible to choose a different scalar field $\phi^{\prime}$ to write $\ell_{a}=\nabla_{a} \phi^{\prime}$ ? Here we demonstrate that this cannot be done in all cases. Of course, one could choose $A$ to be one to start with. But we wish to keep our results general and have hence worked with a general A.

Consider $\ell_{a}=A \nabla_{a} \phi$. Let us assume that this can be written as $\ell_{a}=\nabla_{a} \phi^{\prime}$. Then we would have

$$
\nabla_{a} \ell_{b}-\nabla_{b} \ell_{a}=\nabla_{a} \nabla_{b} \phi^{\prime}-\nabla_{b} \nabla_{a} \phi^{\prime}=0 .
$$

Therefore, the tensor equation $\nabla_{a} \ell_{b}-\nabla_{b} \ell_{a}=0$ should be satisfied. But writing this using the scalar field $\phi$, we have

$$
\nabla_{a} \ell_{b}-\nabla_{b} \ell_{a}=\nabla_{a} A \nabla_{b} \phi-\nabla_{b} A \nabla_{a} \phi=\partial_{a} A \partial_{b} \phi-\partial_{b} A \partial_{a} \phi=0
$$

where the covariant derivatives are just partial derivatives since $A$ and $\phi$ are scalars. It is easy to prove that this equation cannot be satisfied for all choices of $A$ and $\phi$. For example, suppose we consider a four-dimensional spacetime with coordinates $(t, x, y, z)$. Take $\phi=t$. The $t x$-component in the above equation is then

$$
\partial_{t} A \partial_{x} t-\partial_{x} A \partial_{t} t=0 \Rightarrow \partial_{x} A=0
$$

Similarly, we can prove that $\partial_{y} A=0$ and $\partial_{z} A=0$. Thus, in this case, we can write $\ell_{a}=\nabla_{a} \phi^{\prime}$ only in the case where $A=A(t)$. If $A$ depends on any of the other coordinates, this will not be possible. 


\section{Directed surface element on the null surface}

In this appendix, we shall derive the expression for the directed surface element on a null surface. We shall follow the treatment in an appendix of the arxiv version of [12], which itself was inspired from [64], and adapt it to our framework.

Let $y^{\alpha}=\left(y^{1}, y^{2}, \ldots, y^{D-1}\right)$ be any set of coordinates on a codimension-1 null boundary, with associated basis vectors $e_{\alpha}^{a}$. Then, the invariant directed surface element for the surface is given by $[64]$

$$
d \Sigma_{a}=\epsilon_{a a_{1} a_{2} \ldots a_{D-1}} e_{1}^{a_{1}} e_{2}^{a_{2}} \ldots e_{D-1}^{a_{D-1}} d y^{1} d y^{2} \ldots d y^{D-1} .
$$

The contraction of $d \Sigma_{a}$ with any vector on the boundary surface, expressible as a linear combination of $e_{\alpha}^{a}$, is zero because of the antisymmetry of $\epsilon_{a a_{1} a_{2} \ldots a_{D-1}}$. Thus, if $\ell_{a}$ is a null normal to the surface, $d \Sigma_{a}$ must be proportional to $\ell_{a}$ and we may write

$$
\epsilon_{a a_{1} a_{2} \ldots a_{D-1}} e_{1}^{a_{1}} e_{2}^{a_{2}} \ldots e_{D-1}^{a_{D-1}}=f \ell_{a}
$$

for some scalar function $f$. Contracting with the auxiliary null vector $k^{a}$, we obtain

$$
f=-\epsilon_{a a_{1} a_{2} \ldots a_{D-1}} k^{a} e_{1}^{a_{1}} e_{2}^{a_{2}} \ldots e_{D-1}^{a_{D-1}} .
$$

Thus, the directed surface element can be written as

$$
d \Sigma_{a}=-\ell_{a} \epsilon_{b a_{1} a_{2} \ldots a_{D-1}} k^{b} e_{1}^{a_{1}} e_{2}^{a_{2}} \ldots e_{D-1}^{a_{D-1}} d y^{1} d y^{2} \ldots d y^{D-1} .
$$

This is the unique directed surface element on the null surface. There are two ambiguities that one can think of: one being the change of the factor $A$ in the null normal $\ell_{a}=A \nabla_{a} \phi$, the second being the choice of the direction of $k^{a}$ (since there are many null directions to choose from-of all the null directions at a point on the null surface, only the directions along and opposite to $\ell^{a}$ are out of limits for a choice of $k^{a}$ ). It is easy to see that the choice of $A$ does not matter in the above surface element. A scaling of $\ell_{a}$ by changing $A$ will lead to a scaling of $k^{a}$ by the inverse factor to preserve the relation $\ell_{a} k^{a}=-1$. Going on to the second ambiguity, note first that $\left(k^{a}, e_{1}^{a_{1}}, e_{2}^{a_{2}}, \ldots, e_{D-1}^{a_{D-1}}\right)$ forms a basis in the $D$-dimensional spacetime. Any new choice of $k^{a}$, say $k^{\prime a}$, can be expanded in this basis as $k^{\prime a}=k^{a}+\mu_{\alpha} e_{\alpha}^{a}$ for some coefficients $\mu_{\alpha}$. (The coefficient of $k^{a}$ is fixed as one here to ensure $k^{\prime a} \ell_{a}=-1$. We are considering a fixed $\ell_{a}$ here, since we have already shown that the scalings, the only freedom in choosing $\ell_{a}$, do not matter.) But we have

$$
\epsilon_{a a_{1} a_{2} \ldots a_{D-1}} k^{\prime a} e_{1}^{a_{1}} e_{2}^{a_{2}} \ldots e_{D-1}^{a_{D-1}}=\epsilon_{a a_{1} a_{2} \ldots a_{D-1}} k^{a} e_{1}^{a_{1}} e_{2}^{a_{2}} \ldots e_{D-1}^{a_{D-1}}
$$

since the antisymmetry of $\epsilon_{a a_{1} a_{2} \ldots a_{D-1}}$ kills the $\mu_{\alpha} e_{\alpha}^{a}$ part. Thus, we have proved that both the ambiguities we have mentioned do not matter, so that Eq. (125) is the invariant directed surface element on the null surface, independent of our choices for $\ell_{a}$ and $k^{a}$.

The invariant surface element in Eq. (125) is valid for any choice of coordinates $\left(y^{1}, y^{2}, \ldots, y^{D-1}\right)$ on the codimension-1 null boundary. We now consider choices of coordinates for the $D$-dimensional spacetime. Consider an arbitrary set of $D$ coordinates $\left(x^{1}, x^{2}, \ldots, x^{D}\right)$. Then, denoting $k^{x^{1}}=$ $k^{1}, k^{x^{2}}=k^{2}$, etc., we have the scalar density $f$ defined in Eq. (124) in this coordinate system as

$$
f=\sqrt{-g}\left[\begin{array}{ccccc}
k^{1} & k^{2} & k^{3} & \ldots & k^{D} \\
e_{1}^{1} & e_{1}^{2} & e_{1}^{3} & \ldots & e_{1}^{D} \\
e_{2}^{1} & e_{2}^{2} & e_{2}^{3} & \ldots & e_{2}^{D} \\
\vdots & \vdots & \vdots & \ddots & \vdots \\
e_{D-1}^{1} & e_{D-1}^{2} & e_{D-1}^{3} & \ldots & e_{D-1}^{D}
\end{array}\right]
$$

A convenient choice is the set of coordinates $\left(\phi, y^{1}, y^{2}, \ldots, y^{D-1}\right)$, with the null boundary being specified as a constant value of $\phi$. Then the null normal is $\ell_{a}=A \nabla_{a} \phi$ for some $A$. Also, we can 
write $e_{\alpha}^{a}=\partial x^{a} / \partial y^{\alpha}$. (The partial derivative is taken keeping the other coordinates constant. In particular, $\phi$ being constant makes sure that $\partial x^{a} / \partial y^{\alpha}$ at the boundary surface is tangential.) In this coordinate system,

$$
\begin{aligned}
f & =\sqrt{-g}\left[\begin{array}{ccccc}
k^{\phi} & k^{y^{1}} & k^{y^{2}} & \ldots & k^{y^{D-1}} \\
0 & 1 & 0 & \ldots & 0 \\
0 & 0 & 1 & \ldots & 0 \\
\vdots & \vdots & \vdots & \ddots & \vdots \\
0 & 0 & 0 & \ldots & 1
\end{array}\right] \\
& =\sqrt{-g} k^{\phi}=\sqrt{-g} k^{a} \nabla_{a} \phi .
\end{aligned}
$$

Thus, in any coordinate system $\left(\phi, y^{1}, y^{2}, \ldots, y^{D-1}\right)$ with $\phi$ being constant on the null boundary and $\left(y^{1}, y^{2}, \ldots, y^{D-1}\right)$ being arbitrary, we have the surface element in Eq. (125) as

$$
d \Sigma_{a}=-\sqrt{-g} \ell_{a}\left(k^{b} \nabla_{b} \phi\right) d y^{1} d y^{2} \ldots d y^{D-1} .
$$

One could use the condition $\ell_{a} k^{a}=-1$ to write

$$
k^{b} \nabla_{b} \phi=\frac{k^{b} \ell_{b}}{A}=-\frac{1}{A}
$$

so that the surface element becomes

$$
d \Sigma_{a}=\frac{\sqrt{-g}}{A} \ell_{a} d y^{1} d y^{2} \ldots d y^{D-1}
$$

This is a form that we had used in our previous paper [12] as well as in the derivation in this paper. But perhaps Eq. (129) is a more convenient form since it has the $\ell_{a}, k^{a}$ and the coordinate $\phi$, while Eq. (131) has the secondary scalar $A$ that we obtain by expressing $\ell_{a}$ as $A \nabla_{a} \phi$.

In the next section, we discuss the case where $k_{a}$ is chosen as $k_{a}=B \nabla_{a} \Psi$ and the scalar $\Psi$ is chosen as one of the coordinates on the null boundary. This allows us to decompose the determinant of the metric $g$ in terms of the determinant $q$ of the metric on the constant surfaces of $\Psi$ on the null boundary.

Let us briefly digress to note that the derivation till here has not used $\ell_{a} \ell^{a}=0$ or $k_{a} k^{a}=0$, but only $\ell_{a} k^{a}=-1$. Thus, the derivation also works for a non-null surface with appropriate choices of $\ell_{a}$ and $k^{a}$. For example, a spacelike surface with a timelike normal would have the normalized normal satisfying $n_{a} n^{a}=-1$. We can thus choose $\ell_{a}=k_{a}=n_{a}$. Choosing the boundary surface to be a level surface of time $t$, we can write $n_{a}=-N \nabla_{a} t$, where $N$ is the normalization factor and the minus sign has been added so that $n^{a}$ will face in the direction of increasing $t$. Then, in a coordinate system $\left(t, y^{1}, y^{2}, \ldots, y^{D-1}\right)$, the surface element in the form in Eq. (129) would be

$$
\begin{aligned}
d \Sigma_{a} & =-\sqrt{-g} n_{a}\left(n^{b} \nabla_{b} t\right) d y^{1} d y^{2} \ldots d y^{D-1} \\
& =\sqrt{-g} \frac{n_{a}}{N} d y^{1} d y^{2} \ldots d y^{D-1} \\
& =\sqrt{h} n_{a} d y^{1} d y^{2} \ldots d y^{D-1}
\end{aligned}
$$

which is of the familiar form for a spacelike surface (see Appendix B in the arxiv version of [12] for a derivation of $\sqrt{-g}=N \sqrt{h}$ ).

A popular convention is to have the normal vector to be future-directed for spacelike and null surfaces. For the null case, we have provided a comparison of this convention with our convention in Appendix E. 


\section{Directed null surface element with $\phi$ and $\psi$ as coordi- nates}

In this appendix, we shall derive the form of the directed null surface element in the case where the scalars $\phi$ and $\psi$, appearing in $\ell_{a}=A \nabla_{a} \phi$ and $k_{a}=B \nabla_{a} \phi$, are taken as coordinates. (We have further assumed $B=1$ in deriving the null boundary term, but let us work with a general $B$ for the time being.) For this, we need to derive a formula for the determinant of the metric $g$ in terms of the determinant of the metric on the codimension-2 surface, analogous to the formula $g=-N^{2} h$ ( [5], see Appendix B in the arxiv version of [12] for a derivation) connecting the determinant of the metric to the determinant of the induced metric on a spacelike surface. In the last part of Appendix $\mathrm{C}$, we had specialized to a coordinate system $\left(\phi, y^{1}, y^{2}, \ldots, y^{D-1}\right)$. Let us choose $\psi=y^{1}$. Then the codimension- 2 surfaces on the null boundary given by constant values of $\psi$ are the surfaces whose induced metric we have represented as $q_{a b}$. Let the rest of the $D-2$ coordinates be $\left(z^{1}, z^{2}, \ldots, z^{D-2}\right)$. These are the coordinates that run over our codimension- 2 surfaces at the intersection of the level surfaces of $\phi$ and $\psi$. While the small Latin letters $a, b, c, \ldots$ are used as indices to run over all the spacetime coordinates, we shall use the capital Latin letters $A, B, C, \ldots$ to run over only the coordinates $\left(z^{1}, z^{2}, \ldots, z^{D-2}\right)$ corresponding to the codimension-2 surfaces. Let $e_{I}=\partial / \partial z^{I}$, with $I \in\{1,2, \ldots D-2\}$ correspond to the coordinate vectors on the codimension-2 surfaces. We shall write

$$
q_{A B} \equiv g_{a b} \frac{\partial x^{a}}{\partial z^{A}} \frac{\partial x^{b}}{\partial z^{B}}
$$

for the components of the metric on the codimension- 2 surfaces. Then, the metric in such a coordinate system is given by

$$
g_{a b}=\left[\begin{array}{ccccc}
g_{\phi \phi} & g_{\phi \psi} & g_{\phi 1} & \ldots & g_{\phi, D-2} \\
g_{\phi \psi} & g_{\psi \psi} & g_{\psi 1} & \ldots & g_{\psi, D-2} \\
g_{\phi 1} & g_{\psi 1} & q_{11} & \ldots & q_{1, D-2} \\
\vdots & \vdots & \vdots & \ddots & \vdots \\
g_{\phi, D-2} & g_{\psi, D-2} & q_{1, D-2} & \ldots & q_{D-2, D-2}
\end{array}\right],
$$

and the inverse metric is

$$
g^{a b}=\left[\begin{array}{ccccc}
g^{\phi \phi} & g^{\phi \psi} & g^{\phi 1} & \ldots & g^{\phi, D-2} \\
g^{\phi \psi} & g^{\psi \psi} & g^{\psi 1} & \ldots & g^{\psi, D-2} \\
g^{\phi 1} & g^{\psi 1} & q^{11} & \ldots & q^{1, D-2} \\
\vdots & \vdots & \vdots & \ddots & \vdots \\
g^{\phi, D-2} & g^{\psi, D-2} & q^{1, D-2} & \ldots & q^{D-2, D-2}
\end{array}\right] .
$$

From Appendix A.2 in the arxiv version of [12], we have the result that (originally proved there for four dimensions, but the proof can be easily seen to be valid for $D$ dimensions)

$$
g=\frac{q}{g^{\phi \phi} g^{\psi \psi}-\left(g^{\phi \psi}\right)^{2}} .
$$

At the null boundary, the constraints $g^{a b} \ell_{a} \ell_{b}=0, g^{a b} k_{a} k_{b}=0$ and $g^{a b} \ell_{a} k_{b}=-1$ mean the following conditions on the inverse metric, respectively:

$$
g^{\phi \phi}=0, \quad g^{\psi \psi}=0, \quad A B g^{\phi \psi}=-1 .
$$

Substituting this in Eq. (136), we obtain

$$
g=-A^{2} B^{2} q .
$$

Taking the square root,

$$
\sqrt{-g}=A B \sqrt{q} .
$$


which, under our assumption of $B=1$, becomes

$$
\sqrt{-g}=A \sqrt{q} .
$$

Substituting this result in the surface element in Eq. (131), we obtain

$$
d \Sigma_{a}=\sqrt{q} \ell_{a} d \psi d z^{1} \ldots d z^{D-2} \equiv \sqrt{q} \ell_{a} d \psi d^{D-2} z,
$$

where we have set $\left(y^{1}, y^{2}, \ldots, y^{D-1}\right)=\left(\psi, z^{1}, \ldots, z^{D-2}\right)$.

\section{E Translating our results to the future-directed normal con- vention}

In this appendix, we clarify how our convention of choosing the null normal compares with the popular convention of choosing the normal vector to be future-directed (see, e.g., [15]) and specify how our results can be translated to the other convention.

Our conventions for the normal follow those in our earlier paper [12]. The expression obtained after converting the total divergence on the boundary to a surface integral is given in Eq. (24) as

$$
S T=\int_{\partial \mathcal{V}} d^{D-1} \times 2\left(\frac{\sqrt{-g}}{A}\right) \ell_{j} P^{i b j d} \nabla_{b}\left(\delta g_{d i}\right),
$$

where $\ell_{a}$ is defined as $\ell_{a} \equiv A \nabla_{a} \phi$. But the factor $A$ cancels off and we are left with the object $\nabla_{a} \phi$. This is what we obtain if the boundary surface $\phi=$ constant we are considering is such that $\phi$ increases as we move through it from inside the integration volume to the exterior. Thus, the normal is a priori neither future-directed nor past-directed in the conventions of our paper. For any specific example, the nature of the normal would depend on how the scalar $\phi$ is defined.

To make connection with the results in [15], we first make the additional assumption that $\phi$ increases towards the future. Combining this with our assumption that $\phi$ increases as we move from inside the integration volume to the exterior through the boundary surface, moving out through the boundary surface will involve moving forward in time. Since we are working in the $(-,+,+,+)$ convention, the vector corresponding to the normal $\nabla_{a} \phi$ would be past-directed (and inward-directed) at this surface. We can make it future-directed (and outward-directed) by adding an extra minus sign to the definition of the normal vector, as has been done in [15]. Then, the boundary integral becomes

$$
S T=-\int_{\partial \mathcal{V}} d^{D-1} \times 2\left(\frac{\sqrt{-g}}{A}\right) \ell_{j} P^{i b j d} \nabla_{b}\left(\delta g_{d i}\right),
$$

where there is an extra minus sign occurring outside because the definition of $\ell_{j}$ now has an extra minus sign compared to the definition in our paper. If we work in this convention, we will get the same expressions for the boundary terms as in our paper except for overall minus signs.

In the Einstein-Hilbert case, this is why our boundary term differs from that in [15] by a minus sign.

\section{F Deriving the null Raychaudhuri equation from the null projections of the Riemann tensor}

In this appendix, we shall derive the null Raychaudhuri equation [64] with non-affine parametrisation [65] using the projections of the Riemann tensor near a null surface derived in Appendix A. We start with

$$
R_{c}^{a} \ell_{a} \ell^{c}=R_{a b c d} \ell^{a} \ell^{c} g^{b d}=R_{a b c d} \ell^{a} \ell^{c}\left(q^{b d}-k^{b} \ell^{d}-\ell^{b} k^{d}\right)=R_{a b c d} \ell^{a} \ell^{c} q^{b d} .
$$


We take Eq. (115),

$$
R_{a b c d} q_{p}^{a} \ell^{b} q_{q}^{c} \ell^{d}=-q_{p}^{a} q_{q e} £_{\ell} \Theta_{a}^{e}-\Theta_{p}^{e} \Theta_{q e}+\kappa \Theta_{p q},
$$

and contract with $g^{p q}$ to obtain

$$
\begin{aligned}
R_{a b c d} q^{a c} \ell^{b} \ell^{d} & =-q_{e}^{a} £_{\ell} \Theta_{a}^{e}-\Theta_{p}^{e} \Theta_{e}^{p}+\kappa \Theta \\
& =-£_{\ell}\left(q_{e}^{a} \Theta_{a}^{e}\right)+\Theta_{a}^{e} £_{\ell} q_{e}^{a}-\Theta_{p}^{e} \Theta_{e}^{p}+\kappa \Theta \\
& =-£_{\ell}(\Theta)+\Theta_{a}^{e} £_{\ell} q_{e}^{a}-\Theta_{p}^{e} \Theta_{e}^{p}+\kappa \Theta \\
& =-\ell^{a} \nabla_{a} \Theta+\Theta_{a}^{e} £_{\ell} q_{e}^{a}-\Theta_{p}^{e} \Theta_{e}^{p}+\kappa \Theta \\
& =-\ell^{a} \nabla_{a} \Theta+\Theta_{a}^{e}\left(-q_{e}^{c} \nabla_{c} \ell^{a}+q_{c}^{a} \nabla_{e} \ell^{c}\right)-\Theta_{p}^{e} \Theta_{e}^{p}+\kappa \Theta \\
& =-\ell^{a} \nabla_{a} \Theta-\Theta_{a}^{c} \Theta_{c}^{a}+\Theta_{a}^{e} \Theta_{e}^{a}-\Theta_{p}^{e} \Theta_{e}^{p}+\kappa \Theta \\
& =-\frac{d \Theta}{d \lambda}-\Theta_{p}^{e} \Theta_{e}^{p}+\kappa \Theta
\end{aligned}
$$

where we have defined the parameter $\lambda$ varying along the vector $\ell^{a}$ such that $\ell^{a} \nabla_{a} \lambda=1$ (or, equivalently, $\ell^{a}=\partial x^{a} / \partial \lambda$ ) and used the result for the Lie derivative of $q_{e}^{a}$ from Eq. (116):

$$
£_{\ell} q_{b}^{a}=\ell^{c} \nabla_{c}\left(\ell^{a} k_{b}+k^{a} \ell_{b}\right)-q_{b}^{c} \nabla_{c} \ell^{a}+q_{c}^{a} \nabla_{b} \ell^{c} .
$$

From Eq. (144) and Eq. (146), we get the null Raychaudhuri equation:

$$
\frac{d \Theta}{d \lambda}=-R_{c}^{a} \ell_{a} \ell^{c}-\Theta_{b}^{a} \Theta_{a}^{b}+\kappa \Theta
$$

\section{References}

[1] A. Einstein, "Hamilton's Principle and the General Theory of Relativity," Sitzungsber.Preuss.Akad.Wiss.Berlin (Math.Phys.) 1916 (1916) 1111-1116.

[2] C. M. Will, "The Confrontation between General Relativity and Experiment," Living Rev. Rel. 17 (2014) 4, arXiv:1403.7377 [gr-qc].

[3] S. W. Hawking, "The Path Integral Approach To Quantum Gravity," in General Relativity: An Einstein Centenary Survey, pp. 746-789. Univ. Pr., Cambridge, UK, 1979.

[4] E. Dyer and K. Hinterbichler, "Boundary Terms, Variational Principles and Higher Derivative Modified Gravity," Phys.Rev. D79 (2009) 024028, arXiv:0809.4033 [gr-qc].

[5] T.Padmanabhan, Gravitation: Foundations and Frontiers. Cambridge University Press, Cambridge, UK, 2010.

[6] G. Gibbons and S. Hawking, "Action Integrals and Partition Functions in Quantum Gravity," Phys.Rev. D15 (1977) 2752-2756.

[7] J. York, James W., "Role of conformal three geometry in the dynamics of gravitation," Phys.Rev.Lett. 28 (1972) 1082-1085.

[8] J. Katz, "A note on komar's anomalous factor," Classical and Quantum Gravity 2 no. 3, (1985) 423. http://stacks .iop.org/0264-9381/2/i=3/a=018.

[9] J. Katz and D. Lerer, "On global conservation laws at null infinity," Class. Quant. Grav. 14 (1997) 2249-2266, arXiv:gr-qc/9612025 [gr-qc].

[10] N. H. Barth, Second and Fourth Order Gravitational Actions on Manifolds with Boundaries. PhD thesis, The University of North Carolina at Chapel Hill, 1983.

[11] Y. Neiman, "On-shell actions with lightlike boundary data," arXiv:1212.2922 [hep-th]. 
[12] K. Parattu, S. Chakraborty, B. R. Majhi, and T. Padmanabhan, "A Boundary Term for the Gravitational Action with Null Boundaries," Gen. Rel. Grav. 48 no. 7, (2016) 94, arXiv: 1501.01053 [gr-qc].

[13] K. Parattu, S. Chakraborty, and T. Padmanabhan, "Variational Principle for Gravity with Null and Non-null boundaries: A Unified Boundary Counter-term," Eur. Phys. J. C76 no. 3, (2016) 129, arXiv:1602.07546 [gr-qc].

[14] S. Chakraborty, "Boundary Terms of the Einstein-Hilbert Action," Fundam. Theor. Phys. 187 (2017) 43-59, arXiv:1607.05986 [gr-qc].

[15] L. Lehner, R. C. Myers, E. Poisson, and R. D. Sorkin, "Gravitational action with null boundaries," Phys. Rev. D94 no. 8, (2016) 084046, arXiv:1609.00207 [hep-th].

[16] F. Hopfmller and L. Freidel, "Gravity Degrees of Freedom on a Null Surface," Phys. Rev. D95 no. 10, (2017) 104006, arXiv:1611.03096 [gr-qc].

[17] I. Jubb, J. Samuel, R. Sorkin, and S. Surya, "Boundary and Corner Terms in the Action for General Relativity," Class. Quant. Grav. 34 no. 6, (2017) 065006, arXiv:1612.00149 [gr-qc].

[18] S. Aghapour, G. Jafari, and M. Golshani, "On variational principle and canonical structure of gravitational theory in double-foliation formalism," arXiv:1808.07352 [gr-qc].

[19] J. B. Hartle and R. Sorkin, "Boundary Terms in the Action for the Regge Calculus," Gen. Rel. Grav. 13 (1981) 541-549.

[20] E. Farhi, A. H. Guth, and J. Guven, "Is It Possible to Create a Universe in the Laboratory by Quantum Tunneling?," Nucl. Phys. B339 (1990) 417-490.

[21] D. Brill, "Splitting of an extremal Reissner-Nordstrom throat via quantum tunneling," Phys. Rev. D46 (1992) 1560-1565, arXiv:hep-th/9202037 [hep-th].

[22] G. Hayward, "Gravitational action for space-times with nonsmooth boundaries," Phys. Rev. D47 (1993) 3275-3280.

[23] D. Brill and G. Hayward, "Is the gravitational action additive?," Phys. Rev. D50 (1994) 4914-4919, arXiv:gr-qc/9403018 [gr-qc].

[24] A. Reynolds and S. F. Ross, "Complexity in de Sitter Space," Class. Quant. Grav. 34 no. 17, (2017) 175013, arXiv:1706.03788 [hep-th].

[25] R.-Q. Yang, C. Niu, and K.-Y. Kim, "Surface Counterterms and Regularized Holographic Complexity," JHEP 09 (2017) 042, arXiv:1701.03706 [hep-th].

[26] A. Reynolds and S. F. Ross, "Divergences in Holographic Complexity," Class. Quant. Grav. 34 no. 10, (2017) 105004, arXiv:1612.05439 [hep-th].

[27] D. Carmi, R. C. Myers, and P. Rath, "Comments on Holographic Complexity," JHEP 03 (2017) 118, arXiv:1612.00433 [hep-th].

[28] S. Chapman, H. Marrochio, and R. C. Myers, "Complexity of Formation in Holography," JHEP 01 (2017) 062, arXiv:1610.08063 [hep-th].

[29] R.-Q. Yang, "Strong energy condition and complexity growth bound in holography," Phys. Rev. D95 no. 8, (2017) 086017, arXiv:1610.05090 [gr-qc].

[30] O. Ben-Ami and D. Carmi, "On Volumes of Subregions in Holography and Complexity," JHEP 11 (2016) 129, arXiv:1609.02514 [hep-th].

[31] R. Fareghbal and P. Karimi, "Complexity growth in flat spacetimes," Phys. Rev. D98 no. 4, (2018) 046003, arXiv:1806.07273 [hep-th].

[32] R. Auzzi, S. Baiguera, M. Grassi, G. Nardelli, and N. Zenoni, "Complexity and action for warped AdS black holes," JHEP 09 (2018) 013, arXiv:1806.06216 [hep-th]. 
[33] M. Alishahiha, A. Faraji Astaneh, M. R. Mohammadi Mozaffar, and A. Mollabashi, "Complexity Growth with Lifshitz Scaling and Hyperscaling Violation," JHEP 07 (2018) 042, arXiv:1802.06740 [hep-th].

[34] S. Bolognesi, E. Rabinovici, and S. R. Roy, "On Some Universal Features of the Holographic Quantum Complexity of Bulk Singularities," JHEP 06 (2018) 016, arXiv: 1802.02045 [hep-th].

[35] A. P. Reynolds and S. F. Ross, "Complexity of the AdS Soliton," Class. Quant. Grav. 35 no. 9, (2018) 095006, arXiv:1712.03732 [hep-th].

[36] R.-Q. Yang, C. Niu, C.-Y. Zhang, and K.-Y. Kim, "Comparison of holographic and field theoretic complexities for time dependent thermofield double states," JHEP 02 (2018) 082, arXiv:1710.00600 [hep-th].

[37] D. Carmi, S. Chapman, H. Marrochio, R. C. Myers, and S. Sugishita, "On the Time Dependence of Holographic Complexity," JHEP 11 (2017) 188, arXiv: 1709.10184 [hep-th].

[38] R.-Q. Yang, "Complexity for quantum field theory states and applications to thermofield double states," Phys. Rev. D97 no. 6, (2018) 066004, arXiv:1709.00921 [hep-th].

[39] A. R. Brown, D. A. Roberts, L. Susskind, B. Swingle, and Y. Zhao, "Holographic Complexity Equals Bulk Action?," Phys. Rev. Lett. 116 no. 19, (2016) 191301, arXiv:1509.07876 [hep-th].

[40] A. R. Brown, D. A. Roberts, L. Susskind, B. Swingle, and Y. Zhao, "Complexity, action, and black holes," Phys. Rev. D93 no. 8, (2016) 086006, arXiv:1512.04993 [hep-th].

[41] C. Eling, "Spontaneously Broken Asymptotic Symmetries and an Effective Action for Horizon Dynamics," JHEP 02 (2017) 052, arXiv: 1611.10214 [hep-th].

[42] J. Maltz, "de Sitter Harmonies: Cosmological Spacetimes as Resonances," Phys. Rev. D95 no. 6, (2017) 066006, arXiv:1611.03491 [hep-th].

[43] J. Maltz and L. Susskind, "de Sitter Space as a Resonance," Phys. Rev. Lett. 118 no. 10, (2017) 101602, arXiv:1611.00360 [hep-th].

[44] M. Buck, F. Dowker, I. Jubb, and S. Surya, "Boundary Terms for Causal Sets," Class. Quant. Grav. 32 no. 20, (2015) 205004, arXiv:1502.05388 [gr-qc].

[45] C. Lanczos, "A Remarkable property of the Riemann-Christoffel tensor in four dimensions," Annals Math. 39 (1938) 842-850.

[46] D. Lovelock, "The Einstein tensor and its generalizations," J. Math. Phys. 12 (1971) 498-501.

[47] N. Dadhich, "Characterization of the Lovelock gravity by Bianchi derivative," Pramana 74 (2010) 875-882, arXiv:0802.3034 [gr-qc].

[48] T. Padmanabhan and D. Kothawala, "Lanczos-Lovelock models of gravity," Phys. Rept. 531 (2013) 115-171, arXiv:1302.2151 [gr-qc].

[49] N. Deruelle and J. Madore, “On the quasilinearity of the Einstein-'Gauss-Bonnet' gravity field equations," arXiv:gr-qc/0305004 [gr-qc].

[50] S. Chakraborty, "Field equations for Lovelock gravity: An alternative route," Adv. High Energy Phys. 2018 (2018) 6509045, arXiv:1704.07366 [gr-qc].

[51] T. Bunch, "Surface terms in higher derivative gravity," Journal of Physics A: Mathematical and General 14 no. 5, (1981) L139.

[52] R. C. Myers, "Higher Derivative Gravity, Surface Terms and String Theory," Phys.Rev. D36 (1987) 392. 
[53] S. C. Davis, "Generalized Israel junction conditions for a Gauss-Bonnet brane world," Phys. Rev. D67 (2003) 024030, arXiv:hep-th/0208205 [hep-th].

[54] A. Yale, "Simple counterterms for asymptotically AdS spacetimes in Lovelock gravity," Phys. Rev. D84 (2011) 104036, arXiv:1107.1250 [gr-qc].

[55] O. Miskovic and R. Olea, "Counterterms in Dimensionally Continued AdS Gravity," JHEP 10 (2007) 028, arXiv:0706.4460 [hep-th].

[56] N. Deruelle, N. Merino, and R. Olea, "Einstein-Gauss-Bonnet theory of gravity: The Gauss-Bonnet-Katz boundary term," Phys. Rev. D97 no. 10, (2018) 104009, arXiv:1709.06478 [gr-qc].

[57] N. Deruelle, N. Merino, and R. Olea, "Chern-Weil theorem, Lovelock Lagrangians in critical dimensions and boundary terms in gravity actions," arXiv:1803.04741 [gr-qc].

[58] S. Chakraborty, K. Parattu, and T. Padmanabhan, "A Novel Derivation of the Boundary Term for the Action in Lanczos-Lovelock Gravity," Gen. Rel. Grav. 49 no. 9, (2017) 121, arXiv: 1703.00624 [gr-qc].

[59] P. A. Cano, "Lovelock action with nonsmooth boundaries," Phys. Rev. D97 no. 10, (2018) 104048, arXiv:1803.00172 [gr-qc].

[60] T. Padmanabhan, "A short note on the boundary term for the Hilbert action," Mod.Phys.Lett. A29 (2014) 1450037.

[61] D. Lovelock and H. Rund, Tensors, differential forms, and variational principles. Dover Publications, 1989.

[62] T. Frankel, The geometry of physics: an introduction, 2nd edition. Cambridge University Press, 2006.

[63] B. Carter, "The general theory of the mechanical, electromagnetic and thermodynamic properties of black holes," in General Relativity: An Einstein Centenary Survey, S. W. Hawking and W. Israel, eds., ch. 6, pp. 294-369. Cambridge University Press, 1979.

[64] E. Poisson, A Relativist's Toolkit: The Mathematics of Black-Hole Mechanics. Cambridge University Press, 1st ed., 2007.

[65] S. Chakraborty and T. Padmanabhan, "Thermodynamical interpretation of the geometrical variables associated with null surfaces," Phys. Rev. D92 no. 10, (2015) 104011, arXiv: 1508.04060 [gr-qc].

[66] P. Hájičcek, "Exact models of charged black holes," Communications in Mathematical Physics 34 no. 1, (1973) 53-76.

[67] E. Gourgoulhon and J. L. Jaramillo, "A 3+1 perspective on null hypersurfaces and isolated horizons," Phys.Rept. 423 (2006) 159-294, arXiv:gr-qc/0503113 [gr-qc].

[68] A. Corichi, J. D. Reyes, and T. Vukainac, "Weakly Isolated Horizons: First order actions and gauge symmetries," Class. Quant. Grav. 34 no. 8, (2017) 085005, arXiv: 1612.01462 [gr-qc].

[69] P. A. Cano, R. A. Hennigar, and H. Marrochio, "Complexity Growth Rate in Lovelock Gravity," arXiv:1803.02795 [hep-th].

[70] I. Vega, E. Poisson, and R. Massey, "Intrinsic and extrinsic geometries of a tidally deformed black hole," Class. Quant. Grav. 28 (2011) 175006, arXiv:1106.0510 [gr-qc].

[71] R.-Q. Yang and S.-M. Ruan, "Comments on Joint Terms in Gravitational Action," Class. Quant. Grav. 34 no. 17, (2017) 175017, arXiv:1704.03232 [gr-qc].

[72] E. Gourgoulhon, 3+ 1 formalism in general relativity: bases of numerical relativity, vol. 846. Springer Science \& Business Media, 2012.

[73] R. M. Wald, General Relativity. The University of Chicago Press, 1st ed., 1984. 
[74] V. Moncrief and J. Isenberg, "Symmetries of cosmological cauchy horizons," Communications in Mathematical Physics 89 no. 3, (1983) 387-413. http://dx.doi.org/10.1007/BF01214662.

[75] H. Friedrich, I. Racz, and R. M. Wald, "On the rigidity theorem for space-times with a stationary event horizon or a compact Cauchy horizon," Commun.Math.Phys. 204 (1999) 691-707, arXiv:gr-qc/9811021 [gr-qc].

[76] I. Racz, "Stationary Black Holes as Holographs," Class.Quant.Grav. 24 (2007) 5541-5572, arXiv:gr-qc/0701104 [gr-qc].

[77] E. M. Morales, "On a Second Law of Black Hole Mechanics in a Higher Derivative Theory of Gravity," Master's thesis, Institut für Theoretische Physik der Georg-August-Universität zu Göttingen, 2008.

http://www. theorie.physik. uni-goettingen.de/forschung/qft/theses/dipl/Morfa-Morales.pdf.

[78] T. W. Baumgarte and S. L. Shapiro,

Numerical Relativity: Solving Einstein's Equations on the Computer. Cambridge University Press, 2010. 